\title{
Arbeitsgedächtnis und Lernstörungen
}

\author{
Differenzielle Analysen der Funktionstüchtigkeit des
}

Arbeitsgedächtnisses bei Kindern mit Lernstörungen

\author{
Dissertation \\ zur Erlangung des Doktorgrades \\ des mathematisch-naturwissenschaftlichen Doktorgrades \\ „Doctor rerum naturalium“ \\ der Georg-August-Universität Göttingen
}

vorgelegt von

Kirsten Schuchardt

aus Crivitz 
Referent:

Korreferentin:

Tag der mündlichen Prüfung:
Prof. Dr. Marcus Hasselhorn

Prof. Dr. Claudia Mähler

20. Juni 2008 


\section{Inhaltsverzeichnis}

1. Einleitung................................................................... 4

2. Forschungshintergrund...................................................... 6

2.1 Das Konzept des Arbeitsgedächtnismodells von Baddeley .......... 6

2.1.1 Überblick über Baddeleys Arbeitsgedächtnismodell...... 7

2.1.2 Die phonologische Schleife.......................... 7

2.1.3 Der visuell-räumliche Notizblock....................... 10

2.1.4 Die zentrale Exekutive................................ 11

2.2 Lernstörungen................................................ 14

2.2.1 Lese-Rechtschreibstörung.............................. 15

2.2.2 Rechenstörung....................................... 18

2.2.3 Kombinierte Störung schulischer Fertigkeiten............ 21

2.2.4 Lernbehinderung..................................... 22

3. Offene Forschungsfragen und Festlegung der Forschungskriterien....... 25

4. Eigene Analysen............................................................... 30

4.1 Studie 1: Unterscheidet sich die Struktur des Arbeitsgedächtnisses bei Schulkindern mit und ohne Lernstörung?................................ $\quad 30$

4.2 Studie 2: Welche Funktionsbereiche des Arbeitsgedächtnisses sind bei Kindern mit Lese-Rechtschreib- und/oder Rechenstörung beeinträchtigt?

4.3 Studie 3: Zeigen Kinder mit umfassenden Schulleistungsschwierigkeiten in Abhängigkeit von der Intelligenz unterschiedliche Arbeitsgedächtnisdefizite?................................... 56

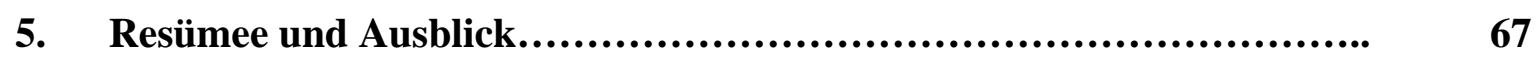

6. Zusammenfassung......................................................... 77

7. Literaturverzeichnis........................................................... $\quad 74$

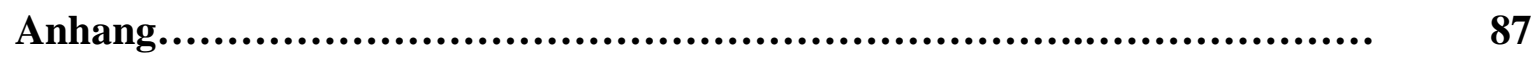




\section{Einleitung}

$\mathrm{Zu}$ den wichtigsten basalen Kompetenzen, die in der Grundschule vermittelt werden, gehören das Lesen, Schreiben und Rechnen. Offensichtlich existieren große interindividuelle Differenzen im Erwerb der schriftsprachlichen und mathematischen Fertigkeiten. Obwohl die meisten Kinder mühelos Lesen, Schreiben und Rechnen erlernen, zeigt ein Teil der Grundschulkinder auch bei guter allgemeiner Lern- und Leistungsfähigkeit und angemessenem Unterricht hierin anhaltende Schwierigkeiten. Angesichts der hohen Auftretenshäufigkeit und Persistenz gewann das Thema Lern- und Leistungsstörungen in den vergangenen Jahren zunehmend an Aufmerksamkeit in Forschung und Praxis. Trotz zahlreicher Forschungsaktivitäten zu möglichen Ursachenfaktoren von Lernstörungen bleiben nach wie vor viele Fragen offen.

Seit einiger Zeit wird die eingeschränkte Funktionstüchtigkeit des Arbeitsgedächtnisses als möglicher Ursachenfaktor für verschiedene Lernstörungen diskutiert und untersucht. Häufig wird dabei das britische Arbeitsgedächtnismodell von Baddeley (1986) zugrunde gelegt. In diesem Modell werden drei Komponenten unterschieden: die modalitätsübergreifende zentrale Exekutive und die zwei ihr untergeordneten kapazitätsbegrenzenden Subsysteme, die phonologische Schleife und der visuell-räumliche Notizblock. Mittlerweile gibt es gut gesicherte Erkenntnisse über den Aufbau und die Funktionsweise des Arbeitsgedächtnisses und dessen Einfluss auf das Lernen und die Schulleistungen. Dagegen ist über die Rolle des Arbeitsgedächtnisses bei Lernstörungen nach wie vor noch wenig bekannt. Die vorliegende Arbeit will zum besseren Verständnis der Informationsverarbeitungsdefizite bei lerngestörten Kindern im Grundschulalter einen Beitrag leisten. Mit einer sehr umfangreichen Testbatterie mit vielen verschiedenen Indikatoren $\mathrm{zu}$ den drei Subkomponenten soll die Funktionstüchtigkeit des Arbeitsgedächtnisses genauer untersucht werden. Gegenstand der Arbeit sind dabei folgende verschiedene Lernstörungen: Kinder mit isolierten Lernstörungen (Lese-Rechtschreibstörung und Rechenstörung) sowie Kinder mit umfassenden Lernstörungen (kombiniert auftretende Lese-Rechtschreib- und Rechenstörung und Lernbehinderung).

Das Ziel der vorliegenden Arbeit besteht in differenziellen Analysen der unterschiedlichen Funktionsbereiche des Arbeitsgedächtnisses bei Kindern mit Lernstörungen, dem in drei separaten Fragestellungen nachgegangen wird. Im ersten Schritt wird die grundlegende Faktorstruktur des Arbeitsgedächtnisses überprüft. Hierbei stellt sich die Frage der Äquivalenz der Struktur des Arbeitsgedächtnisses bei Kindern mit und ohne 
Lernstörungen. Erst bei empirischer Bestätigung der Strukturäquivalenz ist es in der Folge möglich, gefundene Arbeitsgedächtnisbeeinträchtigungen auch als Funktionsdefizite und nicht als Strukturdefizite zu interpretieren. Im zweiten Schritt soll daran anschließend die Rolle der verschiedenen Arbeitsgedächtnisfunktionen bei spezifischen Lernstörungen detailliert analysiert werden. Hierbei wird untersucht, ob Kinder mit Störungen im Lesen, Schreiben und/oder Rechnen Leistungsdefizite in ihren phonologischen, visuell-räumlichen oder zentralexekutiven Arbeitsgedächtnisfunktionen aufzeigen und ob sich für verschiedene Störungsbilder spezifische Defizitmuster aufdecken lassen. Der dritte Schritt dieser Arbeit überprüft die Intelligenz als möglichen kognitiven Ursachenfaktor und verfolgt dabei die Frage, ob sich durchschnittlich intelligente Kinder mit umfassenden Lernschwierigkeiten sowohl im Schriftsprachbereich als auch im Rechnen (kombinierte Störung schulischer Fertigkeiten) von solchen mit gleichzeitig unterdurchschnittlichen intellektuellen Fähigkeiten (Lernbehinderung) hinsichtlich ihrer Gedächtnisfunktionen unterscheiden.

Die vorliegende Untersuchung gliedert sich wie folgt: Im folgenden zweiten Kapitel wird der aktuelle Forschungshintergrund dargestellt. Dabei werden eingangs das theoretische Rahmenmodell des Arbeitsgedächtnisses von Baddeley und Hitch (1974) und etablierte Maße der diagnostischen Erhebung vorgestellt. Anschließend erfolgt eine Zusammenfassung der betrachteten Lernstörungen hinsichtlich Symptomatik, Auftretenshäufigkeit und Stabilität sowie der bisher bekannten ursächlichen kognitiven Funktionsdefizite. Schließlich werden im dritten Kapitel die bislang noch offenen Fragestellungen herausgearbeitet und Forschungskriterien für diese Arbeit festgelegt. Im vierten Kapitel werden die eigenen Analysen dargelegt und im fünften Kapitel die Implikationen der Ergebnisse diskutiert. Mit einer Zusammenfassung der wesentlichen Untersuchungsschritte und der Ergebnisse in Kapitel sechs schließt diese Arbeit. 


\section{Forschungshintergrund}

\subsection{Das Konzept des Arbeitsgedächtnismodell von Baddeley}

Die Vorstellung über das menschliche Gedächtnis veränderte sich stark im letzten Jahrhundert. So führte die Gedächtnisforschung von den Einspeichermodellen zu den so genannten Mehrspeichermodellen, in welchen eine Untergliederung des menschlichen Gedächtnisses in verschiedene Teilsysteme postuliert wird. Den Mehrspeichermodellen liegt die Annahme zugrunde, dass neben dem Langzeitgedächtnis zur langfristigen Informationsspeicherung ein Kurzzeitspeicher existiert, in dem die aktuell verfügbaren Informationen bereitgestellt und verarbeitet werden. Atkinson und Shiffrin (1968) entwickelten in den sechziger Jahren vor dem Hintergrund des Informationsverarbeitungsansatzes ein Gedächtnismodell, in welchem die drei Komponenten sensorisches Register, Kurzzeitgedächtnis und Langzeitgedächtnis hinsichtlich ihrer zeitlichen Speicherung von Information voneinander abgegrenzt werden. Als Verbindungsstück zwischen den Gedächtniskomponenten wird dem Kurzzeitgedächtnis große Bedeutung beigemessen. Atkinson und Shiffrin (1968) bezeichneten es bereits als "Arbeitsgedächtnis", da es selektiv die Informationen aus dem sensorischen Register auswählt, bearbeitet und kurzfristig speichert und somit dem Langzeitgedächtnis zur Verfügung stellt.

Baddeley und Hitch arbeiteten das Konzept des Arbeitsgedächtnisses in den folgenden Jahren weiter aus. In ihrer Konzeption von 1974 gaben sie die Annahme einer einheitlichen Struktur des Arbeitsgedächtnisses auf und beschrieben es im Gegensatz zu Atkinson und Shiffrin als ein dynamisch-aktives System, welches aus mehreren komplex miteinander interagierenden Komponenten besteht. Das ursprüngliche Arbeitsgedächtnismodell wurde in den folgenden Jahren kontinuierlich weiterentwickelt (Baddeley, 1986, 1996, 2000).

Als theoretische Grundlage hat sich das Modell von Baddeley und Hitch in zahlreichen Untersuchungen als eine hilfreiche Grundlage zur Analyse spezifischer Beeinträchtigungen bei Kindern mit Lernstörungen vielfach bewährt (Alloway \& Gathercole, 2006; Pickering, 2006a). So konnte z.B. für die Lese-Rechtschreibstörung und die Rechenstörung gezeigt werden, dass Fehlfunktionen des Arbeitsgedächtnisses zu den Verursachungsfaktoren gehören (Passolunghi, 2006; Swanson, 2006). Baddeleys Arbeitsgedächtnismodell bildet auch für diese Arbeit die theoretische Grundlage, da es durch die differenzierte Beschreibung der Gedächtniskomponenten und -funktionen eine genauere Analyse der basalen kognitiven Funktionen ermöglicht, die im Zusammenhang mit 
Lernstörungen eine Rolle spielen können. Im Folgenden wird es daher ausführlicher dargestellt.

\subsection{1 Überblick über Baddeleys Arbeitsgedächtnismodell}

Im Rahmen des Arbeitsgedächtnismodells von Baddeley und Hitch (1974) werden mehrere Komponenten unterschieden: die modalitätsübergreifende zentrale Exekutive (central executive) sowie zwei modalitätsspezifische Hilfs- bzw. Subsysteme (slave systems) (siehe Abb. 2.1).

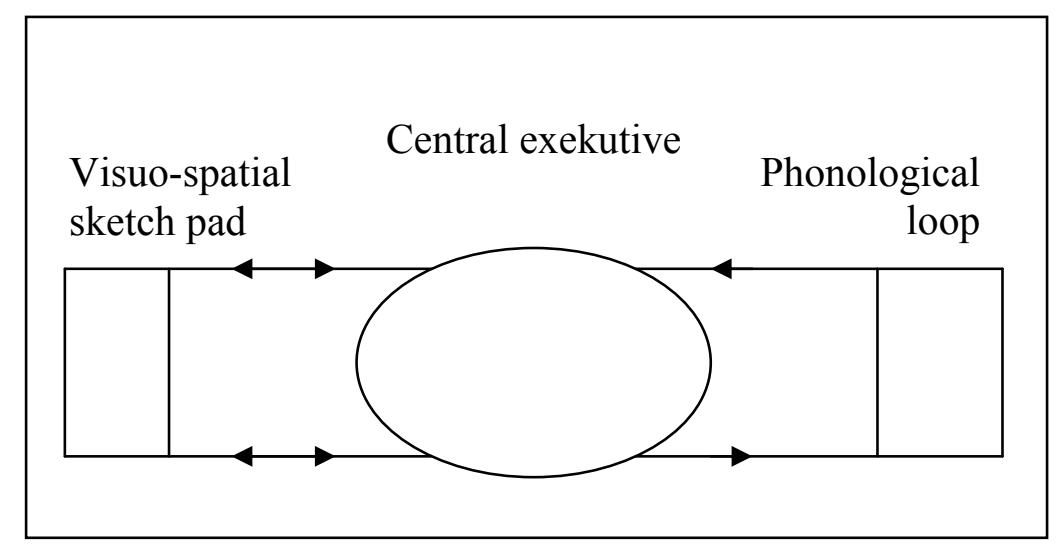

Abbildung 2.1 Darstellung des Arbeitsgedächtnismodells nach Baddeley (1998)

Die im Zentrum stehende zentrale Exekutive stellt eine Art Leitzentrale dar, deren Aufgabe in der Kontrolle und Koordination der zwei ihr untergeordneten kapazitätsbegrenzten Hilfssysteme besteht. Die phonologische Schleife (phonological loop) dient als Hilfssystem zur Aufrechterhaltung und Verarbeitung sprachbasierter Information, der visuell-räumliche Notizblock (visuo-spatial sketchpad) als Hilfssystem zur Aufrechterhaltung und Verarbeitung visueller und räumlicher Information.

\subsubsection{Die phonologische Schleife}

Sprachliche und klangliche Informationen werden in der phonologischen Schleife verarbeitet. Baddeley unterscheidet innerhalb dieses Hilfssystems zwischen zwei Komponenten: dem phonetischen Speicher (phonological store) und dem subvokalen artikulatorischen Rehearsalprozess (subvocal rehearsal). Akustisch-verbales Material erhält unmittelbar Zugang in den phonetischen Speicher. Visuell dargebotene Information muss dagegen erst in einen phonologischen Code übersetzt werden, bevor sie in den phonetischen Speicher gelangt. Die 
phonologische Information kann dort für ca. 1,5 - 2 Sekunden bereitgehalten werden und zerfällt, wenn sie nicht aktiv durch den Prozess des subvokalen Rehearsal, eine Art des inneren Artikulierens, aufgefrischt wird und somit dem phonetischen Speicher weiterhin zur Verfügung steht.

Basierend auf einer Reihe von Untersuchungen nehmen Hasselhorn, Grube und Mähler (2000) eine weitere Ausdifferenzierung der beiden Komponenten der phonologischen Schleife vor. Innerhalb des subvokalen Rehearsalprozesses werden dabei die zwei voneinander abgrenzbaren Funktionsmerkmale, Geschwindigkeit des Rehearsalprozesses und Automatisierungsgrad der Aktivierung, unterschieden. Dem phonetischen Speicher werden die Funktionsmerkmale Größe des Speichers und Verarbeitungspräzision zugeordnet.

Die gesamte Leistungsfähigkeit der phonologischen Schleife hängt sowohl von der Funktionstüchtigkeit des subvokalen Rehearsalprozesses als auch von der des phonetischen Speichers ab (Hasselhorn, Grube \& Mähler, 2000). Als Maß für die funktionale Gesamtkapazität der phonologischen Schleife gilt die verbale Gedächtnisspanne. Zu deren Bestimmung werden Itemfolgen (meist Ziffern oder Wörter) mit anwachsender Länge auditiv vorgegeben, die unmittelbar in der gleichen Reihenfolge wiedergegeben werden sollen. Die maximale Anzahl von Items, die eine Person richtig reproduzieren kann, stellt dabei die individuelle Gedächtnisspanne dar. Die Geschwindigkeit des Rehearsalprozesses kann über die Sprechrate, d.h. die benötigte Zeit zum Artikulieren einer Folge sprachlicher Items (z.B. Topf-Schuh-Baum), geschätzt werden. Empirische Unterstützung erfährt diese Zuordnung durch die konsistent in vielen Untersuchungen gefundene hohe Korrelation zwischen Gedächtnisspanne und Sprechrate (Hasselhorn, Grube \& Mähler, 2000).

Die Funktionstüchtigkeit sowie die Größe der phonologischen Speicherkomponente kann über die Leistung im Nachsprechen von Kunstwörtern (bedeutungsfreie Lautgebilde) erfasst werden (Gathercole, Willis, Baddeley \& Emslie, 1994). Problematisch an dieser Messmethode ist jedoch, dass neben der Kapazität der phonologischen Schleife auch das lexikalische Vorwissen Einfluss auf die Nachsprechleistung hat. Hasselhorn, Seidler-Brandler und Körner (2000) halten diese Aufgabe dennoch zur Erfassung der phonologischen Speicherkapazität für geeignet, da der Einfluss des lexikalischen Vorwissens altersinvariant ist.

Im Folgenden werden einige empirische Befunde und Phänomene im Zusammenhang mit dem Gedächtnisspannenparadigma aufgeführt, die die theoretische Konzeption des Aufbaus und der Arbeitsweise der phonologischen Schleife gut absichern. 
Der Wortlängeneffekt und der Effekt der artikulatorischen Unterdrückung stützen die Annahmen der Komponente des subvokalen Rehearsal. Nach Hasselhorn, Grube und Mähler (2000) dienen diese beiden Phänomene als empirische Indikatoren des Automatisierungsgrades der Aktivierung. Bei Aufgaben zur Gedächtnisspanne von Wörtern kann der Wortlängeneffekt beobachtet werden. Er beschreibt das Phänomen, dass die Gedächtnisspanne für kurze Wörter höher ausfällt als für lange (Baddeley, Thomson \& Buchanan, 1975). Entscheidend ist dabei jedoch weniger die Silbenlänge, als vielmehr die benötigte Zeit zur Aussprache dieser Wörter. Begründet wird dieser Effekt mit der Tatsache, dass beim inneren Artikulieren von längeren Wörtern mehr Zeit benötigt wird und dadurch die Kapazität des phonetischen Speichers bereits mit wenigen Wörtern ausgefüllt ist.

Als weiterer Beleg für die subvokale Rehearsalkomponente gilt der Effekt der artikulatorischen Unterdrückung. Er tritt auf, wenn während der Bearbeitung einer Gedächtnisspannenaufgabe gleichzeitig eine irrelevante Lautfolge (z.B. bla-bla-bla) laut ausgesprochen werden muss. Die serielle Reproduktionsleistung verschlechtert sich unter dieser Bedingung, da durch das laute Artikulieren der Prozess des inneren Artikulierens blockiert wird. Infolgedessen kann die zu behaltende Information nicht aufgefrischt und somit nicht in den phonetischen Speicher eingelesen werden.

Evidenz für die Funktionsweise und Leistungsfähigkeit des phonetischen Speichers ergibt sich aus dem Effekt der irrelevanten Sprache und dem akustischen Ähnlichkeitseffekt. Der Effekt der irrelevanten Sprache beschreibt das Phänomen, dass sich die Leistung einer Gedächtnisspannenaufgabe reduziert, wenn gleichzeitig im Hintergrund sprachlich irrelevantes Material dargeboten wird. Dieser Effekt tritt unabhängig davon auf, ob es sich dabei um eine bekannte Sprache oder um sinnlose unverständliche Silben handelt. Ein gleich bleibendes lautes Rauschen führt dagegen $\mathrm{zu}$ keiner Einbuße in der seriellen Gedächtnisleistung. Diese Befunde führen zu der Annahme, dass das sprachliche Material direkt in den phonetischen Speicher aufgenommen wird und dort mit dem Material aus der Gedächtnisspannenaufgabe interferiert.

Der akustische Ähnlichkeitseffekt besteht darin, dass bei Gedächtnisspannenaufgaben Serien unähnlich klingender Items (z.B. Topf-Schuh-Baum) besser erinnert werden als Serien gleich klingender Items (z.B. Topf-Zopf-Kopf). Die ähnlich klingenden Items sind dabei phonologisch schwerer zu unterscheiden, so dass innerhalb des Speichers die phonologische Kodierung erschwert wird und somit eine niedrigere Gedächtnisspanne resultiert. 
Sowohl der akustische Ähnlichkeitseffekt, der Effekt der irrelevanten Sprache als auch Variationen der Klangqualität während der Darbietung von Kunstwörtern stellen empirische Indikatoren zur Erfassung des funktionalen Merkmals der Verarbeitungspräzision des phonetischen Speichers dar.

\subsubsection{Der visuell-räumliche Notizblock}

Nach Baddeley und Hitch (1974) besteht die Aufgabe des visuell-räumlichen Notizblocks im kurzzeitigen Bereitstellen und Verarbeiten visueller und räumlicher Information. Gab es bislang nur wenige Kenntnisse zu seinem Aufbau und seiner Arbeitsweise, so wurde in jüngerer Zeit verstärkt an der weiteren Ausdifferenzierung dieser Komponente gearbeitet. So kam es zu einer Spezifizierung des visuell-räumlichen Hilfssystems durch Logie (1995), der zwei separate Komponenten unterscheidet, den "visual cache" für visuell-statische und den "inner scribe" für räumlich-dynamische Information (vgl. Pickering \& Gathercole, 2001; Schumann-Hengsteler, Strobl \& Zoelch, 2004).

Der visual cache ist die Speicherkomponente, in der visuelle Merkmale wie Aussehen, Form und Farbe von Objekten verarbeitet werden. Räumliche Informationen wie beispielsweise Lokationen, Relationen und raumzeitliche Abfolgen werden dagegen im inner scribe bereitgehalten. Ähnlich dem subvokalen Rehearsalprozess des phonologischen Arbeitsgedächtnisses findet in diesem Subsystem ein räumlicher Rehearsalprozess statt, der für die Auffrischung von Informationen aus dem visual cache verantwortlich ist. Klassische Aufgaben zur Messung des visuell-räumlichen Gedächtnisses sind beispielsweise die CorsiBlock-Aufgabe (Erfassung der räumlich-dynamischen Komponente) und die Matrix-Aufgabe (Erfassung der räumlich-statischen Komponente).

Gestützt wird die Unterteilung in ein visuelles und ein räumliches Subsystem durch zahlreiche empirische Befunde. Ein Ansatz stellt auch hier das dual-task-Paradigma (Interferenzparadigma) dar. Hierbei erfüllen die Probanden neben einer Primäraufgabe gleichzeitig eine Sekundäraufgabe, die durch Inanspruchnahme der gleichen Arbeitsgedächtnisressourcen die Leistungen der Primäraufgabe beeinträchtigt. Dabei ist die Leistungseinbuße der Primäraufgabe besonders hoch, wenn beide Aufgaben das gleiche Subsystem beanspruchen. Logie und Marchetti (1991) konnten aufzeigen, dass die Leistungen in einer räumlichen Primäraufgabe deutlich durch eine räumliche Interferenzaufgabe (Armbewegungen) und entsprechend die Leistungen in der visuellen Primäraufgabe durch eine visuelle Interferenzaufgabe (irrelevante Bilder) beeinträchtigt werden können. 
Als weiterer Beleg für die Fraktionierung des visuell-räumlichen Notizblocks gelten die von Logie und Pearson (1997) nachgewiesenen unterschiedlichen Entwicklungsverläufe des visuellen und des räumlichen Subsystems. In ihrer Untersuchung bearbeiteten fünf- bis zwölfjährige Kinder die Matrix- und die Corsi-Block-Aufgabe. Die Autoren konnten zeigen, dass, obwohl sich die Leistungen der Kinder in beiden Aufgaben mit zunehmendem Alter verbesserten, die Entwicklung in der visuellen Matrix-Aufgabe schneller verlief. Entsprechende Ergebnisse zu differenten Entwicklungsverläufen der beiden Subkomponenten fanden auch Pickering, Gathercole, Hall und Lloyd (2001).

\subsubsection{Die zentrale Exekutive}

Die zentrale Exekutive ist der Teil des Arbeitsgedächtnisses, welcher bisher am wenigsten konzeptualisiert und untersucht wurde, obwohl Baddeley sie als den wichtigsten und komplexesten Teil des Arbeitsgedächtnismodells versteht. Ursprünglich verglich Baddeley sie mit einem „Lumpensack“, in welchen alle Phänomene, die nicht durch die phonologische Schleife oder den visuell-räumlichen Notizblock erklärbar waren, eingesammelt wurden (Baddeley, 1996). Bei der weiteren Spezifikation der zentralen Exekutive (Baddeley, 1986) orientierte er sich am Modell der Aufmerksamkeitskontrolle von Norman und Shallice (1986). Hierbei ist die zentrale Exekutive in Anlehnung an das "supervisory attentional system" (SAS) der Ort der Aufmerksamkeit und der Kontrolle sowie Steuerung des menschlichen Verhaltens, deren Aufgaben in der Ausarbeitung, Umsetzung und Überwachung von Handlungsplänen besteht. Darüber hinaus ist sie an allen komplexen Leistungen wie schlussfolgerndem Denken und Problemlösen beteiligt. Ungeklärt ist bisher, ob es sich bei der zentralen Exekutive um ein einheitliches System mit verschiedenen Funktionen handelt, oder ob mehrere Systeme mit voneinander unabhängigen Kontrollprozessen unterscheidbar sind.

In der weiteren Differenzierung des Konzepts der zentralen Exekutive grenzte Baddeley (1996) vier verschiedene Funktionen voneinander ab. Die erste Funktion umfasst die zentralexekutive Koordinationskapazität. Treten gleichzeitig vielfältige Anforderungen an das Arbeitsgedächtnis auf, müssen die Informationen aus den beiden Subsystemen parallel verarbeitet und die begrenzten kognitiven Ressourcen verteilt werden. Diese Funktion kann nach Baddeley gut mit der dual-task-Methode untersucht werden, bei der neben einer Hauptaufgabe zusätzlich eine Zweitaufgabe ausgeführt wird. Aufgrund der simultanen Ausführung beider Aufgaben kommt es dabei zu Leistungseinbussen, die auf die Begrenzung der zentralexekutiven Koordinationskapazität hinweisen. 
Die zweite Funktion besteht in der Auswahl und dem Wechsel von verschiedenen Abrufstrategien. Dies ist für die Kontrolle von Handlungen und die Planung und Durchführung von zielgerichtetem Verhalten notwendig. Eine typische Aufgabenanforderung für diese Funktion stellt die Generierung von zufälligen Itemfolgen wie z.B. Buchstaben, Ziffern oder räumlichen Positionen dar. Hierbei müssen gewohnte Itemfolgen (z.B. Alphabet) unterdrückt und die Abrufpläne ständig gewechselt werden.

Als dritte zentralexekutive Funktion führt Baddeley die selektive Aufmerksamkeit an. Gemeint ist damit die Fähigkeit einer Person, die Aufmerksamkeit gezielt auf bestimmte relevante Information bei gleichzeitiger Ausblendung von irrelevanter Information zu richten. Experimentell kann diese Fähigkeit mit dem GO/NOGO-Paradigma erhoben werden. Hierbei sollen die Probanden nur auf Targetitems reagieren, bei irrelevanten Stimuli dagegen keine Reaktionen zeigen.

Der Abruf und die Manipulation von Information aus dem Langzeitgedächtnis ist schließlich die vierte Funktion der zentralen Exekutive. Hierzu gehören der Aufbau, der Erhalt und die Aktivierung von Information des Langzeitgedächtnisses. Somit stellt die zentrale Exekutive das Verbindungsstück zwischen dem Arbeits- und dem Langzeitgedächtnis dar. Zur experimentellen Analyse dieses Aufgabenbereiches wird dabei auf das workingmemory-span Paradigma zurückgegriffen (Daneman \& Carpenter, 1980), bei dem die gleichzeitige Verarbeitung und Speicherung von Informationen gefordert wird. Die Zählspannenaufgabe als komplexes Spannenmaß (Case, Kurland \& Goldberg, 1982), deren Aufgabenanforderung einerseits im Zählen von Kreisen (Verarbeitungskomponente) und andererseits im Behalten der gezählten Kreisanzahlen (Speicherkomponente) besteht, soll hierfür exemplarisch angeführt werden.

Eine weitere Operationalisierung zentralexekutiver Leistungsfähigkeit stellt die Rückwärtsspanne dar, bei der vorgegebene Folgen von Items (z. B. Wörter oder Ziffern) in umgekehrter Reihenfolge unmittelbar wiedergegeben werden sollen (Gathercole \& Pickering, 2000; Hasselhorn \& Schumann-Hengsteler, 2001).

Da der Zusammenhang zwischen Funktionstüchtigkeit des Arbeitsgedächtnisses und Störungen beim Lernen im Mittelpunkt des Interesses der Untersuchung steht, wird im folgenden Abschnitt ein Überblick über die für diese Arbeit relevanten Lernstörungen (LeseRechtschreibstörung, Rechenstörung, kombinierte Störung schulischer Fertigkeiten und 
Lernbehinderung) hinsichtlich ihrer Symptomatik, Auftretenshäufigkeit, Verlauf sowie kognitiver Verursachungsfaktoren gegeben. 


\subsection{Lernstörungen}

Lernstörungen treten bei Schulkindern relativ zahlreich auf und stellen somit eine der häufigsten Ursachen für Schulversagen dar. Unter Lernstörungen werden allgemein Beeinträchtigungen des altersentsprechenden Erwerbs der Kulturtechniken des Lesens, Schreibens und Rechnens verstanden. Eine genaue Begriffsbestimmung ist jedoch nicht einfach, da der Begriff Lernstörungen häufig synonym mit den Bezeichnungen Lernschwierigkeiten, Leistungsversagen, Teilleistungsschwäche und Schulleistungsschwierigkeiten verwendet wird. Nach Klauer und Lauth (1997) können schulische Minderleistungen hinsichtlich ihrer Auftretensdauer unterschieden werden. So treten zeitlich vorübergehende Rückstände häufig als Folge kritischer Lebensereignisse oder situativer Veränderungen wie Schulwechsel, Reifungskrisen oder Interessenänderungen auf. Davon abzugrenzen sind jedoch Lernstörungen, die zeitlich überdauernd sind und sich ohne entsprechende Fördermaßnahmen über die Zeit sogar verstärken. Die Lernstörungen können weiterhin hinsichtlich ihrer Bereichsspezifität in partielle und generelle Lernstörungen differenziert werden (Klauer \& Lauth, 1997). Unter partiellen Lernstörungen werden Minderleistungen in nur einem Lernbereich (z.B. im Schriftsprachbereich) verstanden, während in den anderen schulischen Bereichen unauffällige Leistungen erreicht werden. Um generelle Lernstörungen handelt es sich hingegen, wenn sich die Schwierigkeiten bereichsübergreifend in verschiedenen Leistungsbereichen niederschlagen.

Nach den international anerkannten Diagnosekriterien der gängigen Klassifikationssysteme „Internationale Klassifikation psychischer Störungen“ (ICD-10, 2005) der Weltgesundheitsorganisation (WHO) und „Diagnostisches und Statistisches Manual psychischer Störungen“ (DSM-IV-TR, 2000) der American Psychiatric Association (APA) werden Lernstörungen durch das Vorliegen erwartungswidriger Minderleistungen im Bereich des Lesens, der Rechtschreibung bzw. des Rechnens definiert. Die Leistung soll dabei deutlich unter dem aufgrund des Alters, der allgemeinen Intelligenz und der Beschulung erwartbaren Niveau liegen. Zur Diagnostik wird hierbei dass so genannte doppelte Diskrepanzkriterium herangezogen. Demnach soll erstens die individuelle Schulleistung gemessen über einen standardisierten Schulleistungstest - eine Diskrepanz von mindestens einer Standardabweichung $\mathrm{zu}$ dem für die jeweilige Altersstufe $\mathrm{zu}$ erwartenden Leistungsniveau und damit einen T-Wert unter 40 aufweisen. Zweitens wird zwischen der allgemeinen intellektuellen Leistungsfähigkeit (mit einem Mindest-IQ von 70) und den unterdurchschnittlichen schulischen Leistungen eine bedeutsame Diskrepanz gefordert. Über 
die Größe der kritischen Diskrepanz gibt es bislang keine klaren Festlegungen. Häufig wird eine Differenz von ein bis zwei Standardabweichungen als bedeutsam bewertet (Hasselhorn \& Mähler, 2006). Diesen Kriterien zu Folge werden nicht alle Lernschwierigkeiten als Lernstörungen klassifiziert. So sind Lernstörungen von Lernschwierigkeiten abzugrenzen, bei denen sich die schwachen Schulleistungen nicht signifikant von den intellektuellen Fähigkeiten unterscheiden. Diese Lernschwierigkeiten werden als Lernschwäche bezeichnet. Auch werden schwache Schulleistungen, die im Rahmen einer Lernbehinderung auftreten, von den hier definierten Lernstörungen unterschieden. Kennzeichen dieser Lernbeeinträchtigung besteht darin, dass die schulischen Minderleistungen in verschiedenen Leistungsbereichen gleichzeitig und gemeinsam mit einem unterdurchschnittlichen Intelligenzniveau auftreten und damit die zu erwartende Leistungsstufe darstellen.

Da in Deutschland das Klassifikationssystem der WHO Anwendung findet, orientiert sich auch diese Arbeit an den diagnostischen Kriterien und Einteilungen der ICD-10. Hierin werden Lernstörungen den „Umschriebenen Entwicklungsstörungen schulischer Fertigkeiten“ (F81) zugeordnet. Dabei werden die weiter unterteilten Diagnosen der LeseRechtschreibstörung (F81.0), der Isolierten Rechtschreibstörung (F81.1), der Rechenstörung (F81.2) sowie der Kombinierten Störung schulischer Fertigkeiten (F81.3) unterschieden. Die Kriterien dieser Unterklassifikationen unterscheiden sich bezüglich grundlegender Merkmale nicht voneinander: so werden unangemessene Beschulung, Defizite im Hören und Sehen, neurologische Störungen und psychiatrische Erkrankungen als Verursachung ausgeschlossen. Es wird davon ausgegangen, dass die Ursachen dieser Störungen in Beeinträchtigungen der kognitiven Informationsverarbeitung liegen, die größtenteils auf biologischen Fehlfunktionen beruhen.

\subsubsection{Lese-Rechtschreibstörung}

Kennzeichen der Lese-Rechtschreibstörung ist die umgrenzte Beeinträchtigung der Entwicklung der Lesefertigkeiten und damit häufig verbunden die der Rechtschreibfertigkeiten (ICD-10: F81.0). Einige Kinder haben lediglich Schwierigkeiten im Bereich der Rechtschreibung während die Lesefertigkeiten durchschnittlich ausfallen, so dass hier von einer isolierten Rechtschreibstörung gesprochen wird (ICD-10: F81.1).

Die Probleme im Lesen zeigen sich bereits zu Beginn der Schulzeit in Schwierigkeiten beim Aufsagen des Alphabets und durch Verwechselungen und Verzögerungen beim Benennen von Buchstaben. Betroffen sind das Leseverständnis, die Fähigkeit, gelesene Worte 
wieder zu erkennen und laut vorzulesen. Es treten Lesefehler wie Auslassen, Ersetzen, Verdrehen oder Hinzufügen von Worten oder Wortteilen und Vertauschen von Wortteilen im Satz auf. Besonders auffällig ist eine deutlich reduzierte Lesegeschwindigkeit. Insgesamt beansprucht der Leseprozess sehr viel Anstrengung und Aufmerksamkeit. Die Rechtschreibprobleme sind charakterisiert durch Umstellung der Buchstabenreihenfolge, Auslassungen oder Einfügungen von Buchstaben im Wort, Regelfehler (z.B. Missachtung der Groß/Kleinschreibung, Buchstabenverdoppelung oder Dehnung) und Wahrnehmungsfehler (z.B. Verwechslungen von Lauten wie $\mathrm{d} / \mathrm{t}, \mathrm{b} / \mathrm{p}$ oder $\mathrm{g} / \mathrm{k}$ ). Kennzeichnend für die Rechtschreibprobleme sind weniger typische Fehler als vielmehr eine große Anzahl von Fehlern (Weber \& Marx, 2008). Dabei zeigt sich oftmals eine Fehlerinkonstanz, d.h. ein Wort wird immer wieder unterschiedlich falsch geschrieben.

Epidemiologische Studien zur Auftretenshäufigkeit von Lese-Rechtschreibstörungen ergeben in Abhängigkeit von den eingesetzten Leistungstests und dem verwendetem Diskrepanzkriterium relativ unterschiedliche Schätzungen zwischen $0.5 \%$ und $40 \%$. Unter Zugrundelegung des strengen doppelten Diskrepanzkriteriums bewegen sich die Häufigkeitsangaben von 4\% bis 8\% (Esser \& Schmidt, 1993; Haffner et al., 1998; Lewis, Hitch \& Walker, 1994; Miles, Haslum \& Wheeler, 1998; Rutter et al., 2004; Shaywitz, Escobar, Shaywitz, Fletcher \& Makuch, 1992). Dabei zeigt sich eine Altersabhängigkeit in der Prävalenzschätzung, nach der 7-8\% der achtjährigen Kinder, 6\% der zwölfjährigen Kinder und nur noch 4\% im jungen Erwachsenenalter von einer Lese-Rechtschreibstörung betroffen sind (Hasselhorn \& Schuchardt, 2006). Das Sinken der Prävalenz im Laufe des Kindes- und Jugendalters scheint sowohl durch Spontanremissionen als auch durch Auswirkungen eingesetzter Fördermaßnahmen erklärbar zu sein (Hasselhorn \& Schuchardt, 2006). Hinsichtlich der Geschlechterverteilung zeigt sich über verschiedene Studien übereinstimmend ein erhöhtes Auftreten von Lese-Rechtschreibschwierigkeiten zu Ungunsten der Jungen. Dabei liegen die Prävalenzschätzungen in klinisch anfallenden Stichproben bei einem 5.9-fach (Finucci \& Childs, 1981) bzw. 2- bis 3-fach erhöhten Risiko (Flannery, Liederman, Daly \& Schultz, 2000; Miles et al., 1998; Rutter et al., 2004) für Jungen im Vergleich zu Mädchen. Nach Kontrolle aller potentiell die Jungen benachteiligenden Faktoren wie größere Anpassungsprobleme oder erhöhte Neigung zu Aufmerksamkeits- und anderen Verhaltensproblemen ist die Prävalenz der Lese-Rechtschreibstörung bei Jungen etwa 1.74bis 2-mal so hoch wie bei Mädchen (Liederman, Kantrowitz \& Flannery, 2005; vgl. Hasselhorn \& Schuchardt, 2006). 
Längsschnittlich angelegte Studien zeigen eine zeitlich hohe Stabilität umschriebener Lese- und Rechtschreibstörungen oft bis ins Erwachsenenalter (vgl. Hasselhorn \& Schuchardt, 2006). Die betroffenen Kinder verbessern sich zwar absolut in ihren Schriftsprachleistungen, bleiben aber dennoch im Vergleich zu ihren unbeeinträchtigten Mitschülern zurück. Shaywitz et al. (1992) und Francis et al. (2005) schätzen LeseRechtschreibstörungen als persistierend ein, da in ihrer Studie von den Kindern, die in der dritten Klasse die Kriterien einer Lese-Rechtschreibstörung erfüllen, 78\% auch in der 5. Klasse noch Minderleistungen im Lesen aufweisen und etwa 59\% weiterhin die Diagnose Lese-Rechtschreibstörung erfüllen. In einer Untersuchung von Strehlow, Kluge, Möller und Haffner (1992) erfüllten 58 von 59 ursprünglich in einer kinder- und jugendpsychiatrischen Ambulanz als lese-rechtschreibgestört diagnostizierte Patienten auch noch knapp 13 Jahre später die Kriterien und zwar unabhängig davon, ob sie eine entsprechende Therapie erhalten hatten oder nicht. Esser und Schmidt (1993) fanden dagegen nur bei einem Drittel der Kinder, bei denen mit 8 Jahren eine Lese-Rechtschreibstörung diagnostiziert wurde, nach 5 Jahren eine deutliche Besserung der Symptomatik. Ähnlich fielen die Ergebnisse von Schulte-Körne, Deimel, Jungermann und Remschmidt (2003) aus. In ihrer Studie erfüllten 37\% der Kinder, die eine hessische Spezialschule für Legasthenie besuchten, auch noch nach 20 Jahren die Kriterien einer Lese-Rechtschreibstörung.

Als Ursachen der Lese-Rechtschreibstörung werden genetische und hirnorganische Faktoren, ungünstige Umweltbedingungen, Persönlichkeitsmerkmale und kognitive Faktoren diskutiert (Weber \& Marx, 2008). Da die verschiedenen Ursachen nicht bei jedem Kind gleichermaßen eine Rolle spielen, führt dies möglicherweise $\mathrm{zu}$ dem sehr heterogenen Störungsbild der Lese-Rechtschreibschwierigkeiten. In der Ursachenforschung wurde der Suche nach kognitiven Defiziten bislang besondere Aufmerksamkeit geschenkt. Hierbei konzentrierten sich die Forschungsbemühungen zunehmend auf die Defizite in der phonologischen Verarbeitung und Speicherung. So ist heute unumstritten, dass es sich bei der Lese-Rechtschreibstörung um eine phonologische Informationsverarbeitungsstörung handelt (Pickering, 2006b; Swanson, 2006; Vellutino, Fletcher, Snowling \& Scanlon, 2004). Unter phonologischer Informationsverarbeitung wird die Nutzung von Informationen über die Lautstruktur bei der Auseinandersetzung mit gesprochener oder geschriebener Sprache verstanden (Wagner \& Torgesen, 1987). Diese lässt sich weiter in die drei Faktoren phonologische Bewusstheit, phonologisches Rekodieren im Arbeitsgedächtnis und Abruf phonologischer Codes aus dem Langzeitgedächtnis aufgliedern. Eine Vielzahl von Befunden aus dem angloamerikanischen Sprachraum führten zur Formulierung der Doppel-Defizit- 
Hypothese, nach der Lesebeeinträchtigungen sowohl mit Defiziten in der phonologischen Bewusstheit als auch mit Beeinträchtigung in der Geschwindigkeit beim Abruf phonologischer Codes einhergehen (Wolf \& Bowers, 1999). Im deutschen Sprachraum, in dem die Schwierigkeiten der betroffenen Kindern im Gegensatz zum angloamerikanischen Raum weniger die Lesegenauigkeit, sondern vielmehr die Lesegeschwindigkeit und die Rechtschreibung betreffen, lassen sich zusätzlich Hinweise auf einen Einfluss des phonologischen Arbeitsgedächtnisses aufdecken. Auch in Längsschnittstudien konnte gezeigt werden, dass spätere Lese- und Rechtschreibleistungen durch alle drei Komponenten vorhergesagt werden können (Klicpera \& Gasteiger-Klicpera, 1998; Näslund \& Schneider, 1996; Schneider \& Näslund, 1993), so dass in der deutschen Sprache das DreiKomponentenmodell der phonologischen Informationsverarbeitung Gültigkeit hat (vgl. Hasselhorn \& Gold, 2006). Eine Vielzahl von Studien hat sich mit den Schwierigkeiten leserechtschreibschwacher Kinder bei der Bearbeitung von Gedächtnisanforderungen beschäftigt, so dass mittlerweile zu allen drei Bereichen des Arbeitsgedächtnisses Forschungsergebnisse vorliegen. Eine Beeinträchtigung des phonologischen Arbeitsgedächtnisses leserechtschreibschwacher Kinder konnte in mehreren Studien nachgewiesen werden (Helland \& Asbjørnsen, 2004; Schuchardt, Kunze, Grube \& Hasselhorn, 2006). Auch finden sich Hinweise auf defizitäre zentralexekutive Funktionen (Landerl, Bevan \& Butterworth , 2004; Palmer, 2000; Pickering \& Gathercole, 2005; Schuchardt et al., 2006; Siegel \& Ryan, 1989; Swanson, 1993, 1999). Beeinträchtigungen in visuell-räumlichen Arbeitsgedächtnisleistungen werden bei leseschwachen Kindern dagegen eher selten berichtet (Eden \& Stein, 1995; Howes, Bigler, Burlingame, \& Lawson, 2003; Kibby, Marks, Morgan, \& Long, 2004; O'Shaughnessy \& Swanson, 1998; Pickering, 2006b).

\subsubsection{Rechenstörung}

Laut ICD-10 liegen die Schwierigkeiten rechengestörter Kinder vor allem in der mangelnden Beherrschung der Grundrechenarten Addition, Subtraktion, Multiplikation und Division, nicht jedoch in den höheren mathematischen Fertigkeiten (z.B. Algebra, Trigonometrie, Geometrie und Differential- sowie Integralrechnung). Wesentlich für alle betroffenen Kinder mit Schwierigkeiten im Rechnen ist, dass sie keine typischen Fehler aufweisen. Vielmehr unterscheiden sie sich von den mathematisch unauffälligen Kindern durch die hohe Anzahl von Fehlern und nicht durch deren Qualität (Dockrell \& McShane, 1993). Dabei können die auftretenden Schwierigkeiten sehr verschiedenartig sein und nur in einem oder auch in 
mehreren der folgenden Bereiche auftreten. Mögliche Fehlerquellen sind ein Nichtverstehen der zugrundeliegenden mathematischen Konzepte wie Zahlbegriff oder auch Zahlraum, ein mangelndes Verständnis mathematischer Ausdrücke oder Symbole, ein Nichtwiedererkennen numerischer Zeichen sowie Schwierigkeiten beim Ausführen von Standardrechenschritten, im Anwenden mathematischer Erkenntnisse in Sachsituationen, beim Schreiben von Zahlen und Symbolen in der richtigen Reihenfolge. Auch ein Mangel beim räumlichen Aufbau von Berechnungen und Schwierigkeiten beim Erlernen des Einmaleins können zu fehlerhaften Rechnungen führen (vgl. WHO, 2005; Schulz, 1995).

Die aktuellen Prävalenzangaben zum Auftreten einer Rechenstörung liegen zwischen 5-8 \% (im Überblick Hasselhorn und Schuchardt, 2006). Allerdings legen die vorhandenen epidemiologischen Studien verschiedener Länder zur Bestimmung von Minderleistungen im Rechnen unterschiedliche Kriterien zugrunde. So zeigt sich in Studien, in denen ein Leistungsrückstand in Mathematik von mindestens zwei Schuljahren bei gleichzeitig durchschnittlicher Intelligenz als Definitionskriterium zugrunde gelegt wurde, eine Auftretenshäufigkeit von 5.5\% bei einer indischen Stichprobe (Ramaa \& Gowramma, 2002) und 6.5\% bei einer israelischen Stichprobe (Gross-Tsur, Manor \& Shalev, 1996). Deutlich niedriger fallen die Ergebnisse einer englischen Studie von Lewis et al. (1994) aus, die bei 910-jährigen Schulkindern eine Prävalenz von 3.6\% feststellten. Die wenigen Studien, die das strenge doppelte Diskrepanzkriterium angewendet hatten, kommen mit 6\% (Mazzocco \& Myers, 2003) bzw. 4.3-5.3\% (Fuchs, Compton, Fuchs, Paulsen, Bryant \& Hamlett, 2005) in etwa zu ähnlichen Einschätzungen. Das Geschlechterverhältnis wird im Gegensatz zur LeseRechtschreibschwäche, bei der die Anzahl der Jungen deutlich überwiegt, als relativ ausgeglichen angegeben (Gross-Tsur et al., 1996; Lewis et al., 1994; Ramaa \& Gowramma, 2002).

Zusammenfassend stellt Grube (2008) übergeordnete Problembereiche dar, in denen Schüler mit Rechenstörung massive Schwierigkeiten zeigen. So werden rechenspezifische Merkmale und rechenunspezifische Merkmale von einander unterschieden. Zu den grundlegenden rechenspezifischen Problemen zählen Defizite in den numerischen Basisfähigkeiten. Diese fundamentalen numerischen Kompetenzen als direkte Vorläuferfunktionen des Rechnens bestehen aus verschiedenen Aspekten zum Umgang mit Zahlen wie dem Aufbau des Zahlenbegriffs oder der Beherrschung des Zählens. Während bei Aufgaben zur Zahlenproduktion und zu Zahlenkonzepten rechenschwache Kinder keine oder nur wenige Defizite aufweisen, können nach Geary (2004) Beeinträchtigungen in den Fertigkeiten des Zählens und dem sicheren Zahlenumgang beobachtet werden. Es zeigt sich, 
dass die von Gelman und Gallistel (1978) beschriebenen Zählprinzipien der Eins-zu-EinsZuordnung, der Kardinalität und der stabilen Reihenfolge der Zahlwörter beim Zählen grundsätzlich verstanden werden. Dennoch treten bei entsprechenden Aufgaben häufig Fehler auf. Demgegenüber scheint bei schwachen Rechnern das Wissen des Prinzips der irrelevanten Reihenfolge unvollständig ausgebildet zu sein. In Untersuchungen mit rechenschwachen Grundschulkindern (Geary et al., 1999; 2000) stellte sich heraus, dass das Abzählen einer Menge von Objekten in einer ungeordneten Reihenfolge von den betroffenen Kindern als falsch beurteilt wurde. Die rechenschwachen Kinder scheinen überzeugt davon zu sein, dass die Reihenfolge der zu zählenden Objekte nicht beliebig sein dürfe, sondern eine wichtige Eigenschaft des Zählens darstelle. Ein weiterer Problembereich kann unter dem Begriff basale Rechenfertigkeiten zusammengefasst werden (Grube, 2008). Hierzu zählt das Anwenden unreifer Rechenstrategien. So vollziehen rechenschwache Grundschulkinder über lange Zeit nicht die typische Entwicklung von zähl- zu gedächtnisbasierten Rechenstrategien sondern benutzen häufig Strategien, die für jüngere Kinder ohne Beeinträchtigungen im Rechnen charakteristisch sind (Geary, 1999). Bei der Anwendung der einfachen Zählstrategien unterlaufen ihnen dabei jedoch viele Fehler. Infolgedessen kann das arithmetische Wissen nur unvollständig und unsystematisch aufgebaut werden, so dass ein automatischer Abruf grundlegender Fakten erschwert wird, und die betroffenen Kinder nach wie vor auf zählbasierte Rechenstrategien zurückgreifen müssen.

Aufgrund mangelnder Studien zur Stabilität von Rechenstörungen gibt es über deren Verlauf bisher nur wenig gesicherte Kenntnisse. Nach einer Einschätzung von Shalev und Gross-Tsur (2001) kann man Aussagen über den Verlauf von Lese-Rechtschreibschwächen auch auf die Rechenschwierigkeiten übertragen. Demnach ist davon auszugehen, dass Störungen im Rechnen oftmals nicht nur eine Entwicklungsverzögerung sondern ein langfristiges Problem darstellen. Shalev, Manor, Auerbach und Gross-Tsur (1998) schätzen Rechenschwächen als zeitlich überdauernd ein, da in ihrer Studie mehr als 47\% der Kinder, die in der fünften Klasse Schwächen im Rechnen aufzeigen, diese auch noch in der achten Klasse besitzen. In der Untersuchung von Mazzocco und Myers (2003) zeigten sogar 63\% der Kinder mit diagnostizierter Rechenstörung eine Persistenz ihrer Schwierigkeiten über einen Zeitraum von mindestens zwei Jahren auf.

Als wichtigste rechenunspezifische Ursache ist neben der allgemeinen kognitiven Leistungsfähigkeit die Beeinträchtigung der allgemeinen Gedächtnisfähigkeit zu nennen (Grube, 2008). So äußert sich beispielsweise eine reduzierte Gedächtniskapazität in Schwierigkeiten beim Erinnern basaler arithmetischer Fakten aus dem Langzeitgedächtnis 
oder auch in einer fehlenden Speicherung von Zwischenergebnissen, was wiederum zu Problemen im Rechnen führen kann. Für Kinder mit Minderleistungen im Rechnen zeigt sich hinsichtlich der Arbeitsgedächtnisbeeinträchtigungen bislang ein sehr heterogenes Forschungsbild (Passolunghi, 2006). So finden sich viele Hinweise, dass nicht alle Arbeitsgedächtnisfunktionen in gleicher Weise Defizite aufweisen. Die Ergebnisse der verschiedenen Studien zeigen vornehmlich im Bereich der zentralen Exekutive Beeinträchtigungen rechenschwacher Kinder (Gaupp, 2003; Geary et al., 1999, 2000; Hitch \& McAuley, 1991, McLean \& Hitch, 1999; Passolunghi \& Siegel, 2001; Swanson, 1993; Swanson \& Sachse-Lee, 2001; Wilson \& Swanson, 2001). Dagegen werden phonologische (Grube \& Barth, 2004; Hitch \& McAuley, 1991; Schuchardt et al., 2006; Swanson \& SachseLee, 2001) und visuell-räumliche Auffälligkeiten (Gaupp, 2003; McLean \& Hitch, 1999) der betroffenen Kinder nur vereinzelt berichtet.

\subsubsection{Kombinierte Störung schulischer Fertigkeiten}

Kommt es zu einem gemeinsamen Auftreten einer Störung sowohl im LeseRechtschreibbereich als auch im Rechnen wird nach ICD-10 die Diagnose Kombinierte Störung schulischer Fertigkeiten (F81.3) gestellt. Hier liegen die Lernschwierigkeiten auf breiter Ebene, d.h. im Lesen, Rechtschreiben und Rechnen bei gleichzeitig guter Intelligenz. Somit ist diese Störung zu unterscheiden von der Lernbehinderung, bei der ebenfalls deutlich unterdurchschnittliche Leistungen in den verschiedenen Leistungsbereichen auftreten jedoch mit einer unterdurchschnittlichen intellektuellen Begabung einhergehen.

Verschiedene epidemiologische Studien zeigen auf, dass ein beträchtlicher Anteil der Schüler gleichzeitig Leistungsbeeinträchtigungen in den Bereichen des Rechnens sowie des Lesens und Schreibens hat. Gross-Tsur et al. (1996) berichten, dass $17 \%$ der rechenschwachen Kinder gleichzeitig Schwächen im Lesen und 7.5\% Schwächen im Lesen und Schreiben aufweisen. Lewis et al. (1994) ermittelten sogar eine höhere Prävalenz der kombinierten Störung von 2.3\% im Gegensatz zu einer Auftretenswahrscheinlichkeit einer isolierten Rechenstörung von 1,3\%.

Nach der Einschätzung von Hasselhorn und Schuchardt (2006) gibt es hinsichtlich des Geschlechterverhältnisses ein interessantes Phänomen. Da Jungen ein höheres Risiko für das Auftreten einer Lese-Rechtschreibstörung aufzeigen und bei Rechenstörungen das Risiko für Jungen und Mädchen in etwa gleich ist, sollte demzufolge das Risiko für das kombinierte Auftreten der beiden Lernstörungen bei Jungen erhöht, wenn auch weniger deutlich als bei 
der isolierten Lese-Rechtschreibstörung, ausfallen. Erstaunlicherweise findet sich aber ein Geschlechterverhältnis von 1:1.3 zwischen Jungen und Mädchen. Dieser Befund legt die Vermutung nahe, dass es sich beim kombinierten Auftreten von Lese-Rechtschreib- und Rechenstörungen möglicherweise um eine funktional eigenständige Lernstörung handelt.

$\mathrm{Zu}$ kognitiven Verursachungen bei Kindern mit kombinierten Leistungsstörungen liegen bisher nur sehr wenige Erkenntnisse vor. Dies liegt hauptsächlich daran, dass die beiden Leistungsbereiche bislang nur getrennt voneinander untersucht wurden. Erst in jüngster Zeit zeigt sich ein zunehmendes Forschungsinteresse auch an dieser Lernstörung. Als ursächliche Faktoren werden hier vor allem gemeinsame kognitive Defizite, insbesondere Gedächtnisdefizite, vermutet. Studien, die Kinder mit kombinierten Störungen mit isoliert beeinträchtigten Kindern vergleichen, machen deutlich, dass bei diesen Kindern besonders gravierende Arbeitsgedächtnisdefizite vorliegen (Andersson \& Lyxell, 2007; Geary et al., 1999, 2000; Siegel \& Ryan 1989; van der Sluis, van der Leij, \& de Jong, 2005). In einer eigenen Untersuchung zu Kindern mit schwachen Rechen-, schwachen LeseRechtschreibleistungen oder kombiniert schwachen Leistungen im Rechnen, Lesen und Schreiben konnte kein Hinweis auf ein unabhängiges charakteristisches Defizitmuster in den Arbeitsgedächtnisleistungen nachgewiesen werden (Schuchardt et al., 2006). Ähnliche Ergebnisse berichten auch van der Sluis et al. (2005). Es ist jedoch noch offen, ob diese Schlussfolgerung auch für Kinder mit einer klinisch relevanten Kombinierten Störung schulischer Fertigkeiten zutrifft.

\subsubsection{Lernbehinderung}

Kennzeichnend für Kinder mit Lernbehinderung ist, dass sie trotz spezifischer Förderung in der Regelschule keine ausreichenden Lernfortschritte machen und dies auch zukünftig nicht zu erwarten ist (Laut \& Schlottke, 2005). Es handelt sich demnach nicht um eine Lernstörung im strengen Sinne nach den oben aufgeführten Kriterien der ICD-10. Vielmehr findet sich das Konzept der Lernbehinderung lediglich im deutschen Schulsystem wieder und entspringt einer schulorganisatorischen Definition (Deutscher Bildungsrat, 1973). Unter Lernbehinderung wird eine überdauernde und umfassende Beeinträchtigung der Lern- und Leistungsfähigkeit verstanden, die mit einer Vielzahl von Symptomen einhergeht. Die betroffenen Kinder verfügen über verminderte intellektuelle Fähigkeiten, die ein bis drei Standardabweichungen unter dem Mittelwert der jeweiligen Alterstufe liegen (dies entspricht IQ-Werten zwischen 55 und 85). Hierbei kommt es zu einer Überschneidung mit der 
Diagnose einer geistigen Behinderung, die nach den Kriterien der ICD-10 (F70) durch einen IQ $<70$ gekennzeichnet ist. Folgende Merkmale sind charakteristisch für lernbehinderte Kinder: Erstens bestehen Rückstände in verschiedenen Lernbereichen (v.a. in Deutsch und Mathematik), zweitens liegt der Leistungsstand mehr als zwei Jahre hinter dem der Mitschüler zurück, drittens bestehen die Minderleistungen über mehrere Jahre und können nicht durch spezifische Förderung beseitigt werden, viertens treten umfangreiche kognitive, soziale und sprachliche Entwicklungsrückstände auf und fünftens können die Minderleistungen nicht auf eine Sinnesschädigung zurückgeführt werden (Lauth \& Schlottke, 2005). Es kommt somit zu einem Zusammenwirken von Minderleistungen und Minderbegabung, in deren Folge die betroffenen Kinder nicht die gesetzten Lernziele der entsprechenden Alters- und Klassenstufe erreichen. Lernbehinderte Kinder lernen wesentlich langsamer und insgesamt weniger, vergessen schneller die gelernten Inhalte, weisen größere Schwierigkeiten beim abstrakten Begriffslernen auf und können gelernte Inhalte schlechter auf neue Situationen transferieren (Grünke, 2004). Die Folge sind oftmals fehlende Lernerfolge auch nach Klassenwiederholungen und der Wechsel auf eine entsprechende Förderschule.

Die Prävalenzrate liegt gemäß der Definition unter der Annahme der Normalverteilung der Intelligenzwerte bei $16 \%$ der Schülerschaft. Wenngleich nicht alle hierunter fallenden Schüler einen sonderpädagogischen Förderbedarf mit Schwerpunkt Lernen benötigen, so ist dennoch der Anteil der betroffenen Kinder sehr hoch (vgl. Kretschmann, 2007). Nach Angaben der Kultusministerkonferenz betrug der Anteil der schulpflichtigen Kinder und Jugendlichen mit sonderpädagogischen Förderbedarf im Schwerpunkt Lernen im Jahr 2002/03 bundesweit 2,93\%, wobei etwa 88\% der Betroffenen in entsprechenden Förderschulen und $12 \%$ an allgemein bildenden Schulen unterrichtet wurden (Sekretariat der Ständigen Kultusministerkonferenz, 2004). Verglichen mit den Angaben der vorherigen Jahre ist ein stetiges Anwachsen der bedürftigen Kinder $\mathrm{zu}$ verzeichnen. Jungen sind insgesamt häufiger als Mädchen von Lernbehinderung betroffen. Es zeigt sich ein Verhältnis von 3:2 zu Ungunsten der Jungen (Lauth \& Schlottke, 2005). Auch findet sich ein erhöhter Anteil von Schülern aus sozial benachteiligten Verhältnissen (80-90\%) und von Schülern mit Migrationshintergrund (17\%; vgl. Grünke, 2004).

Obwohl sich die Definition der Lernbehinderung lediglich auf die Schulzeit beschränkt, bleiben deutliche Folgen fürs weitere Leben sichtbar (Laut \& Schlottke, 2005). So verließen im Schuljahr 2002/2003 insgesamt etwa 9\% der Schüler die Schule ohne einen Hauptschulabschluss, von denen knapp die Hälfte (4\% der Gesamtstichprobe) eine Sonderschule und 2,6\% eine Schule mit Förderschwerpunkt Lernen besuchten (Statistisches 
Bundesamt, 2004). Das Leistungsniveau der lernbehinderten Schüler liegt deutlich unter dem der Kinder mit Hauptschulabschluss und entspricht zu Schulende etwa dem der 4. bis 6 . Klassenstufe einer Regelschule (Wocken, 2000).

Die Lernbehinderung wird durch das Zusammenwirken multipler Faktoren erklärt. Diskutiert werden einerseits in der Umwelt liegende Ursachenbedingungen. Hierzu zählen ungünstige schulische Lernbedingungen und Sozialisationsfaktoren (anregungsarme Elternhäuser, ungünstige sozioökonomische Bedingungen wie beispielsweise niedriges Einkommen, niedriger Berufsstatus der Eltern oder auch beengte Wohnverhältnisse; Grünke, 2004). Andererseits werden auch biologische Faktoren (genetische Defekte, Geburtskomplikationen oder Infektionen) angenommen. Zudem existieren Hinweise auf eine ganze Reihe kognitiver Funktionsdefizite, zu denen hauptsächlich Beeinträchtigungen in der Informationsverarbeitung (z.B. Aufmerksamkeit, Arbeitsgedächtnis), ein eingeschränktes strategisches Verhalten beim Lernen, ein unzureichendes bereichsspezifisches Vorwissen, ein Mangel an metakognitiven Fertigkeiten sowie eine verminderte Motivation zählen (Lauth \& Schlottke, 2005; Grünke, 2004). Da die aufgeführten spezifischen Merkmale nicht immer gleichermaßen auftreten, umfasst die Gruppe der Kinder mit Lernbehinderungen insgesamt einen äußerst heterogenen Schülerkreis.

Zur Beschreibung und Erklärung kognitiver Bedingungen lernbehinderter Kinder wurde im Rahmen der Developmental-Difference-Kontroverse (Zigler \& Balla, 1982) eine Vielzahl von Studien zu der Frage durchgeführt, ob der Verlauf ihrer kognitiven Entwicklung im Vergleich zu lernunbeeinträchtigten Kindern nur zeitlich verzögert sei oder ob Unterschiede in der kognitiven Struktur zwischen den beiden Gruppen zu finden sind. Hierzu wurden auch Gedächtnisleistungen einer genaueren Analyse unterzogen. Bisherige empirische Studien belegen insgesamt umfassende Beeinträchtigungen in allen drei Subkomponenten des Arbeitsgedächtnisses (Gathercole \& Pickering, 2001; Henry, 2001; Henry \& MacLean, 2002; Mähler, 2007; Pickering \& Gathercole, 2004; van der Molen, van Luit, Jongmans \& van der Molen, 2007). Dabei scheint die Leistungsfähigkeit der phonologischen Schleife und der zentralen Exekutive besonders massiv beeinträchtigt zu sein (Henry \& MacLean, 2002; Mähler \& Hasselhorn, 2003; Hasselhorn \& Mähler, 2007; Rosenquist, Conners \& RoskosEwoldsen, 2003; van der Molen et al., 2007). Da sich in einigen Studien Bestätigung für die Hypothese der zeitlich verzögerten Entwicklung fand, in anderen Studien jedoch dem widersprechend strukturelle Abweichungen von der normalen Gedächtnisentwicklung beobachtet wurden, ist die Frage nach den kognitiven Besonderheiten dieser Kinder bis heute noch nicht hinreichend geklärt. 


\section{Offene Forschungsfragen und Festlegung der Forschungskriterien}

In den vorangegangenen Kapiteln wurde ein Überblick über das Modell des Arbeitsgedächtnisses und die in dieser Arbeit betrachteten Lernstörungen gegeben. Dabei wurde deutlich, dass die verschiedenen Lernstörungen aufgrund der hohen Auftretenshäufigkeit und des oftmals ungünstigen Verlaufs ein ernstzunehmendes Problem darstellen. Einen viel versprechenden Ansatz zur Erklärung von Lernstörungen stellt die Untersuchung kognitiver Defizite der betroffenen Kinder dar. Ausgehend vom Arbeitsgedächtnismodell von Baddeley sollen durch eine differenzierte Analyse der Arbeitsgedächtniskomponenten und -funktionen die kognitiven Grundlagen von Lernstörungen näher untersucht werden. Hierzu werden die Leistungen von Grundschulkindern mit verschiedenen Lernstörungen (Lese-Rechtschreibstörung, Rechenstörung, Kombinierte Störung schulischer Fertigkeiten und Lernbehinderung) mit Hilfe einer umfassenden Testbatterie zum Arbeitsgedächtnis analysiert. Folgenden bislang noch offenen Fragestellungen soll im Rahmen dieser Arbeit nachgegangen werden:

1) Ein erstes Ziel besteht in der Untersuchung der grundlegenden Arbeitsgedächtnisstruktur und verfolgt dabei die Frage, ob sich die Struktur des Arbeitsgedächtnisses bei Schulkindern mit und ohne Lernstörung unterscheidet. In der Literatur findet sich eine Vielzahl von Arbeiten, die bei Kindern mit schulischen Minderleistungen schwache Arbeitsgedächtnisleistungen nachweisen. Diese werden in der Regel als Funktionsdefizite im Arbeitsgedächtnis diskutiert. Diese Interpretation ist jedoch nur zulässig, wenn das Arbeitsgedächtnis der lernbeeinträchtigten und das der lernunbeeinträchtigten Kinder eine äquivalente Struktur besitzt. Sollte sich andererseits die Gedächtnisstruktur der beiden Gruppen voneinander unterscheiden, kann nicht ausgeschlossen werden, dass die gefundenen Leistungsunterschiede auf qualitative Strukturdefizite der betroffenen Kinder zurückzuführen sind.

In einer Reihe von Studien an unausgelesenen Stichproben konnte eine unabhängige Funktionsweise der verschiedenen Subsysteme und damit die Gültigkeit des Arbeitsgedächtnismodells empirisch nachgewiesen werden. Mittels Strukturanalysen ließ sich das Vorhandensein eines phonologischen und eines visuell-räumlichen Subsystem bereits bei Vorschulkindern im Alter von vier und fünf Jahren bestätigen (Roebers \& Zoelch, 2005; Schmid, Zoelch \& Roebers, 2008). Ab einem Alter von sechs Jahren lässt sich erstmals die dreigliedrige Arbeitsgedächtnisstruktur weitestgehend belegen (Alloway, Gathercole \& 
Pickering, 2006; Gathercole, Pickering, Ambridge \& Wearing, 2004). In der Literatur finden sich allerdings auch Hinweise auf Zwei-Komponentenmodelle. So finden Gathercole und Pickering (2000) bei Kindern mit 7 Jahren ein Modell, welches lediglich zwischen der phonologischen Schleife und der zentralen Exekutive unterscheidet. Auch Jarvis und Gathercole (2003) identifizieren ein zweigliedriges Modell, in dem zwischen verbalen und nonverbalen Aufgabenanforderungen unterschieden wird, welches dem DreiKomponentenmodell in der Anpassungsgüte deutlich überlegen ist. Somit sind die Ergebnisse hinsichtlich der empirischen Strukturanalyse bislang sehr heterogen. Weiterführende Analysen hierzu stellen ein weiteres Anliegen dieser Studie dar.

Die Überprüfung der Vorraussetzung der Äquivalenz zwischen der bei unausgelesenen Stichproben nachgewiesenen Faktorenstruktur und der Struktur bei Kindern mit verschiedenen Lern- und Leistungsstörungen sowie ein Vergleich zwischen einem Drei- und einem Zwei-Komponentenmodell des Arbeitsgedächtnisses stehen somit im Interesse der ersten Studie dieser Arbeit, die in Kapitel 4.1 ausführlich dargestellt wird.

2) Weiterhin ist von Interesse, ob partielle Besonderheiten des Arbeitsgedächtnisses bei Kindern mit klinisch relevanten Lernstörungen bestehen. Wie oben aufgeführt ist die Befundlage $\mathrm{zu}$ den spezifischen Arbeitsgedächtnisbeeinträchtigungen insgesamt recht uneinheitlich. Studien mit lese-rechtschreibschwachen Kindern belegen deutliche Schwierigkeiten in phonologischen Arbeitsgedächtnisaufgaben (Pickering, 2006b; Swanson, 2006; Vellutino, 2004). Die Untersuchungen zur Erfassung der zentralen Exekutive und zum visuell-räumlichen Notizblock kommen dagegen zu widersprüchlichen Ergebnissen. Auch bei Kindern mit schwachen Rechenleistungen zeigt sich ein heterogenes Befundmuster. So findet sich in mehreren Untersuchungen eine Beeinträchtigung der zentralen Exekutive (Geary et al., 1991; Geary et al., 1999, 2000; Hitch \& McAuley, 1991; McLean \& Hitch, 1999; Passolunghi \& Siegel, 2001; Siegel \& Ryan, 1989; Swanson, 1993; Swanson \& Sachse-Lee, 2001; Wilson $\&$ Swanson, 2001). Eher selten lassen sich auch in der phonologischen Schleife (Geary et al., 1991; Hitch \& McAuley, 1991; Schuchardt et al., 2006; Swanson \& Sachse-Lee, 2001) und im visuell-räumlichen Notizblock (Reuhkala, 2001; McLean \& Hitch, 1999; van der Sluis et al., 2005) schwache Arbeitsgedächtnisleistungen rechenbeeinträchtigter Kinder aufdecken. Gründe hierfür liegen möglicherweise in der geringen Vergleichbarkeit durch die verschiedenen untersuchten Stichproben, den unterschiedlich strengen Auswahlkriterien und den verwendeten Testverfahren zur Bestimmung von Lernstörungen. Darüber hinaus wurden 
die verschiedenen Arbeitsgedächtnisfunktionen oftmals nur durch wenige und recht unterschiedliche Aufgaben operationalisiert. Es ist also unklar, ob die beschriebenen Befunde auch auf Kinder mit umschriebenen Lernstörungen zutreffen. Zu Kindern mit kombinierten Leistungsschwächen gibt es bislang nur sehr wenige Untersuchungen (Geary et al., 1999, 2000; Schuchardt et al., 2006; Siegel \& Ryan 1989; van der Sluis et al., 2005). Da die betroffenen Kinder jeweils in einer Vielzahl von Aufgaben $\mathrm{zu}$ den verschiedenen Arbeitsgedächtniskomponenten schwächere Leistungen aufzeigten als Kinder mit isolierten Schwächen, scheint bei ihnen ein generelles Defizit im Arbeitsgedächtnis vorzuliegen. Weitere Analysen zu den kognitiven Besonderheiten dieser Kinder stehen bislang noch aus.

Die zweite Studie der vorliegenden Arbeit verfolgt daher konkret die Frage, welche Funktionsbereiche des Arbeitsgedächtnisses bei Kindern mit Störungen im Rechnen und/oder im Lesen und Schreiben beeinträchtig sind (Kap. 4.2). Hierzu werden Kinder mit Rechenstörung und/oder Lese-Rechtschreibstörung hinsichtlich ihrer phonologischen, visuellräumlichen und zentralexekutiven Arbeitsgedächtnisfunktionen untersucht. Dabei soll insbesondere geklärt werden, ob das Auftreten von Rechen- und/oder LeseRechtschreibstörungen mit voneinander unterscheidbaren Arbeitsgedächtnisdefizitmustern einhergeht. Durch die Berücksichtigung sowohl der Kinder mit isolierten Lernstörungen, d.h. Lese-Rechtschreibstörung und Rechenstörung, als auch der Kinder mit einer kombinierten Lernstörung innerhalb eines Untersuchungsdesigns wird ein Vergleich hinsichtlich der Spezifität von Gedächtnisdefiziten ermöglicht.

3) Als drittes soll der Frage nachgegangen werden, ob Kinder mit umfassenden Schulleistungsschwierigkeiten in Abhängigkeit von der Intelligenz unterschiedliche Arbeitsgedächtnisdefizitmuster aufzeigen. Wie oben dargelegt wurde, werden Kinder mit massiven schulischen Minderleistungen in der Schriftsprache und im Rechnen zwei voneinander unterscheidbaren Gruppen, nämlich Kinder mit zur Intelligenz diskrepanten Schulleistungsschwierigkeiten (kombinierte Störung schulischer Fertigkeiten) und Kinder mit gleichzeitig unterdurchschnittlichen intellektuellen Fähigkeiten (Lernbehinderung), zugeordnet. Die hinter der Diskrepanzdefinition stehende Annahme besagt, dass die Intelligenz als verursachender Faktor für schwache Schulleistungen ausgeschlossen wird und andere kognitive Funktionsbeeinträchtigungen, wie z.B. spezifische Funktionsstörungen des Arbeitsgedächtnisses, als Erklärung vermutet werden. Bei Kindern mit Lernbehinderungen 
wird dagegen gerade die niedrige intellektuelle Leistungsfähigkeit als ein erklärendes Merkmal für die schulischen Minderleistungen gesehen.

Als mögliche gemeinsame kognitive Defizite werden bei Kindern mit generellen Lernproblemen Gedächtnisschwierigkeiten gesehen. Wie in den aufgeführten Studien deutlich wurde, zeigen Kinder mit Lernbehinderung umfassende Beeinträchtigungen im Arbeitsgedächtnis. Auch bei Kindern mit einer kombinierten Störung schulischer Fertigkeiten existieren Hinweise auf Schwierigkeiten in allen drei Subkomponenten. Es ist es jedoch bislang unklar, ob Kinder mit Lernbehinderung gleiche Arbeitsgedächtnisdefizite aufzeigen wie Kinder mit einer kombinierten Lernstörung. Hier setzt die dritte Studie (Kap. 4.3) dieser Arbeit an, in der Erkenntnisse darüber gewonnen werden sollen, inwieweit sich Funktionsdefizite des Arbeitsgedächtnisses bei gleichermaßen schwachen Schulleistungen jedoch verschiedenen Ausprägungsgraden des Intelligenzniveaus unterscheiden.

Die vorliegende Arbeit bildet somit eine wichtige Erweiterung bisheriger Untersuchungen in diesem Themenfeld. Die in den folgenden Kapiteln beschriebenen Studien stammen aus dem seit 2001 bis zum gegenwärtigen Zeitpunkt laufenden Forschungsprojekt Funktionsstörungen im Arbeitsgedächtnis bei Kindern der Abteilung für Pädagogische Psychologie und Entwicklungspsychologie der Universität Göttingen. Die Rekrutierung der Kinder mit Lernstörungen erfolgte in Zusammenarbeit mit der abteilungseigenen Beratungsstelle für Lernschwierigkeiten, Teilleistungsstörungen und Hochbegabung. Dort werden u.a. Kinder mit Verdacht auf Lernstörungen vorgestellt und einer intensiven Individualdiagnostik unterzogen. Die Kinder mit diagnostizierter Lernstörung nehmen zusätzlich am Forschungsprojekt teil und werden an einem separaten Termin hinsichtlich ihrer Arbeitsgedächtnisleistungen untersucht. Die Kinder der Kontrollgruppe stammen aus einer unausgelesenen Stichprobe von Grundschulkindern. Hierzu wurden sechs Grundschulklassen hinsichtlich der intellektuellen Fähigkeiten, der Leistungen in den schulischen Bereichen Lesen, Schreiben und Rechnen sowie des Arbeitsgedächtnisses untersucht und die für die Parallelisierung jeweils geeigneten Kontrollkinder ausgewählt. Die eingesetzten Testverfahren und Arbeitsgedächtnisaufgaben werden in den einzelnen Studien detailliert beschrieben.

Um Mängel früherer Studien zu überwinden und eine vergleichbare hohe Qualität zwischen den drei Studien zu gewährleisten, werden übergeordnete und einheitliche 
Forschungskriterien für diese Arbeit definiert. Hierzu werden folgende Festlegungen getroffen:

a) Die Arbeitsgedächtnisleistungen werden mit einer sehr umfangreichen Testbatterie mit vielen verschiedenen Indikatoren zu den drei Subkomponenten detailliert untersucht.

b) Die diagnostische Bestimmung der Lernstörungen erfolgt ausschließlich über standardisierte Schulleistungstests und Intelligenztestverfahren mit aktueller Normierung.

c) Es findet eine Einschränkung des Altersbereiches auf das Grundschulalter statt.

d) Kinder mit unzureichenden Deutschkenntnissen werden aus den Untersuchungen ausgeschlossen.

e) Die Auswahl der klinischen Stichproben wird sehr sorgfältig nach strengen Kriterien durchgeführt.

Für die Entwicklungsstörungen schulischer Fertigkeiten (ICD-10) gelten: (1) IQ $\geq 80$, (2) die Lese-Rechtschreib- und/oder Rechenleistungen sind deutlich unterdurchschnittlich $(\mathrm{T}<40)$ und (3) besteht eine kritische Diskrepanz zwischen dem Intelligenzniveau und $\begin{array}{lllll}\text { den unterdurchschnittlichen } & \text { Schulleistungen von mindestens } & 1.2\end{array}$ Standardabweichungen.

Für die Lernbehinderung gilt: (1) IQ<85, (2) die Lese-Rechtschreib- und Rechenleistungen sind deutlich unterdurchschnittlich und entsprechen damit den intellektuellen Fähigkeiten $(\mathrm{T}<40)$.

f) Die Leistungen der Kinder mit verschiedenen Lernstörungen werden einer nach Alter, Geschlecht und weitestgehend Intelligenz parallelisierten Kontrollgruppe gegenübergestellt.

Für die Kontrollgruppe gilt: (1) IQ>85, (2) mindestens durchschnittliche Leistungen in allen untersuchten schulischen Leistungsbereichen $(\mathrm{T}<40)$.

g) Zur besseren Vergleichbarkeit werden nur Kinder in die Untersuchungen einbezogen, die eine Regelgrundschule besuchen.

Es folgt im Kapitel 4 die getrennte Darstellung der drei durchgeführten Studien zur Beantwortungen der aufgeworfenen Fragestellungen anhand von Untersuchungsdesigns, die den oben genannten Forschungskriterien Folge leisten. 


\section{Eigene Analysen}

\subsection{Studie 1: Unterscheidet sich die Struktur des Arbeitsgedächtnisses bei Schulkindern mit und ohne Lernstörung? ${ }^{1}$}

Das Arbeitsgedächtnis dient der vorübergehenden Speicherung und Verarbeitung von Informationen. Seine Funktionstüchtigkeit hat sich als guter Prädiktor für den Erfolg von Lernprozessen erwiesen. Das gilt sowohl für den Erwerb der Sprache (vgl. Hasselhorn \& Werner, 2000) als auch für den Erwerb von Lesen, Schreiben und Rechnen (vgl. Grube \& Hasselhorn, 2006). Im Bereich der Lernschwierigkeiten gibt es mittlerweile zahlreiche Hinweise, dass spezifische Lernstörungen mit Fehlfunktionen im Arbeitsgedächtnis einhergehen (z.B. Alloway \& Gathercole, 2006; Pickering, 2006; Schuchardt, Mähler \& Hasselhorn, in press). Daher rührt ein zunehmendes Interesse an einer Funktionsdiagnostik des Arbeitsgedächtnisses (vgl. Gathercole, Pickering, Ambridge \& Wearing, 2004; Hasselhorn et al., 2003).

Obwohl sehr unterschiedliche Modelle des Arbeitsgedächtnisses vorgelegt wurden, wird in entwicklungspsychologischen und pädagogisch-psychologischen Arbeiten zunehmend auf das in den letzten 30 Jahren von Baddeley $(1986,2006)$ immer weiter elaborierte britische Arbeitsgedächtnismodell Bezug genommen. Die Speicherungs- und Verarbeitungsfunktionen werden diesem Modell zufolge durch zwei Hilfssysteme erfüllt, die von einem übergeordneten System - der zentralen Exekutive - überwacht und gesteuert werden. Akustisch-sprachliche Information wird in der sog. ,phonologischen Schleife“ verarbeitet und gespeichert, während ein ,visuell-räumlicher Notizblock“ auf die Verarbeitung und Speicherung von visuellen und räumlichen Informationen spezialisiert ist. Das übergeordnete System der zentralen Exekutive leistet unterschiedliche Funktionen der Selektion und Koordination von Informationen und verfügbaren Ressourcen und bildet damit die Verarbeitungsgrundlage bei der Bewältigung komplexer kognitiver Anforderungen.

In einer Reihe von Studien konnte die Unabhängigkeit der verschiedenen Subsysteme des Arbeitsgedächtnisses demonstriert werden. Roebers und Zoelch (2005) wiesen das Vorhandensein eines phonologischen und eines visuell-räumlichen Subsystems bereits ab einem Alter von ca. vier Jahren nach. Spätestens ab dem Alter von sechs Jahren bis ins hohe Erwachsenenalter lässt sich eine dreigliedrige Arbeitsgedächtnisstruktur identifizieren

\footnotetext{
1 Dieses Kapitel basiert auf einer Publikation, die in der Zeitschrift für Entwicklungspsychologie und Pädagogische Psychologie erschienen ist (Schuchardt, K., Roick, T., Mähler, C. \& Hasselhorn, M. (2008) Unterscheidet sich die Struktur des Arbeitsgedächtnisses bei Schulkindern mit und ohne Lernstörung?).
} 
(Alloway, Gathercole \& Pickering, 2006; Gathercole et al., 2004; Kane et al., 2004). Insgesamt scheint die Struktur des Arbeitsgedächtnisses altersinvariant zu sein, da sie über alle Altersgruppen in gleicher Weise nachweisbar ist. Dies spricht dafür, dass die nach Subsystemen differenzierte Erfassung des Arbeitsgedächtnisses im Zusammenhang mit der Differenzialdiagnostik bei Kindern mit Lernstörungen ab etwa vier Jahren angemessen zu sein scheint.

Aufgrund der nachgewiesenen Altersinvarianz der Arbeitsgedächtnisstruktur werden in der einschlägigen Literatur die berichteten Arbeitsgedächtnisdefizite von Kindern mit Lernund Leistungsstörungen in der Regel als spezifische Funktionsdefizite interpretiert. Diese Interpretation setzt voraus, dass Kinder mit Lern- und Leistungsstörungen über die gleiche prinzipielle Arbeitsgedächtnisstruktur verfügen, wie sie bei unausgelesenen Stichproben gefunden wurde (Struktur-Äquivalenz-Hypothese). Bis heute ist die Struktur-ÄquivalenzHypothese nicht geprüft worden. Denkbar wäre daher auch, dass die Arbeitsgedächtnisdefizite solcher Kinder nicht auf ein spezifisches Funktionsdefizit zurück zu führen sind, sondern darauf, dass diese Kinder über eine andere Struktur des Arbeitsgedächtnisses verfügen als unauffällige Kinder. Die empirische Prüfung der StrukturÄquivalenz-Hypothese war ein Anliegen der hier vorgelegten Untersuchung.

\section{Methode}

Versuchspersonen. 186 Erst- bis Viertklässler, die in einem Zeitraum von vier Jahren in der Beratungsstelle für Lernschwierigkeiten an der Universität Göttingen vorstellig wurden, sowie eine unausgelesene Stichprobe von 59 Grundschulkindern nahmen an der umfangreichen diagnostischen Untersuchung teil. Die Intelligenz der Kinder wurde über die K-ABC erfasst. Mit den Tests der WRT-Reihe wurde die Rechtschreibleistung erhoben, mit dem Salzburger Lesetest (SLT) die Leseleistung und mit den Tests der DEMAT-Reihe die Rechenleistung. Auf Basis dieser Testdaten wurde bei insgesamt 129 Kindern (76 Jungen, 53 Mädchen) mit einem Durchschnittsalter von 108.27 Monaten $(S D=11.39)$ eine Lernstörung diagnostiziert: Nach ICD-10 Kriterien erhielten 65 Kinder (50.39\%) die Diagnose LeseRechtschreibstörung (F81.0), 10 Kinder (7.75\%) die der isolierten Rechtschreibstörung (F81.1), 19 Kinder (14.73\%) die der Rechenstörung (F81.2) und 22 Kinder (17.05\%) die der kombinierten Störung schulischer Fertigkeiten (F81.3). Weitere 13 Kinder (10.08\%) wurden als lernbehindert (Intelligenz unter dem Durchschnittsbereich) klassifiziert. Die Gruppe der Kinder ohne Lernstörung ( $n=116$ : 46 Jungen, 70 Mädchen) mit einem Durchschnittsalter von 
108.21 Monaten $(S D=12.76)$ umfasst Kinder mit einem IQ $\geq 85$ und unauffälligen Schulleistungen $(\mathrm{PR}>15)$ in allen untersuchten Bereichen.

Arbeitsgedächtnis-Testbatterie. Die Funktionstüchtigkeit des Arbeitsgedächtnisses wurde mit einer umfangreichen Testbatterie von insgesamt 9 Aufgaben untersucht. Zur Erfassung der phonologischen Schleife wurden die Ziffernspanne, die Wortspanne für einund dreisilbige Wörter sowie das Kunstwortnachsprechen eingesetzt. Bei den Spannenaufgaben wurden ansteigende Sequenzen von Ziffern (1-9) bzw. einsilbige (z.B. Topf, Eis, Stern) oder dreisilbige Wörter (z.B. Eisenbahn, Erdbeere) akustisch vorgegeben, die unmittelbar in der gleichen seriellen Reihenfolge wiederzugeben waren. Beim Kunstwortnachsprechen wurden insgesamt 24 bedeutungsfreie zwei-, drei- und viersilbige Kunstwörter (z.B. maling, jalosse, fallurwakel) nach akustischer Darbietung anschließend einzeln korrekt nachgesprochen. Es wurde ein Gesamtmaß aus der Anzahl richtig reproduzierter Wörter bestimmt.

Der visuell-räumliche Notizblock wurde mittels Corsi-Block-Aufgabe und einer Matrix-Aufgabe erfasst. Für die Bearbeitung der Corsi-Block-Aufgabe stand ein Brett der Größe 23 x $27.5 \mathrm{~cm}$ zur Verfügung, auf welchem 9 Klötzchen in unregelmäßiger Anordnung befestigt sind. Im Sekundenrhythmus werden Sequenzen von Blöcken unterschiedlichen Komplexitätsniveaus mit dem Finger angetippt, die anschließend von dem Kind in der gleichen Reihenfolge nachgezeigt werden sollen. Bei der Matrix-Aufgabe wurden Sequenzen von weißen und schwarzen Mustern unterschiedlicher Komplexität simultan in einer 4x4 Matrix am Computer präsentiert. Unmittelbar nach der Präsentation sollten die Muster in einer leeren Matrix erinnert werden.

Die zentral-exekutiven Arbeitsgedächtnisfunktionen wurden über die Ziffern- und Wortspanne rückwärts sowie die Zählspanne erhoben. Bei den Rückwärtsspannenaufgaben müssen Folgen von Ziffern bzw. einsilbigen Wörtern nicht wie bei den Vorwärtsspannen in der vorgegebenen, sondern in umgekehrter Reihenfolge wiedergegeben werden. In der Zählspanne werden am Computer ansteigende Sequenzen von Vorlagen zufällig angeordneter Kreise und Vierecke präsentiert, wobei das Kind die Kreise zählen und sich die Anzahl merken soll. Nach Abschluss einer Sequenz sollten die gemerkten Einzelergebnisse in der richtigen Reihenfolge wiedergegeben werden.

Die Durchführung und Bewertung erfolgte bei allen Gedächtnisspannenaufgaben gleichermaßen. Es wurden stets anwachsende Sequenzen beginnend mit zwei bis maximal 
acht Items vorgegeben. Die längste korrekt wiedergegebene Sequenzlänge stellt die individuelle Gedächtnisspanne der jeweiligen Aufgabe dar.

\section{Ergebnisse}

\section{Deskriptive Daten und Korrelationsmatrix}

Tabelle 4.1.1 enthält für die Versuchsgruppen und die Gesamtgruppe die Mittelwerte, Standardabweichungen und Gruppenvergleiche zu den einzelnen Arbeitsgedächtnisaufgaben.

Tabelle 4.1.1 Deskriptive Statistiken (Mittelwerte und Standardabweichungen) und Gruppenvergleich

\begin{tabular}{lcccc}
\hline \multicolumn{1}{c}{ Arbeitsgedächtnisbatterie } & $\begin{array}{c}\text { Gesamtgruppe } \\
(n=245)\end{array}$ & $\begin{array}{c}\text { ohne Lernstörung } \\
(n=116)\end{array}$ & $\begin{array}{c}\text { mit Lernstörung } \\
(n=129)\end{array}$ & $d$ \\
\hline Ziffernspanne & $4.87(0.80)$ & $5.11(0.79)$ & $4.67(0.77)$ & $0.57^{*}$ \\
Wortspanne, 1-silbig & $4.41(0.75)$ & $4.58(0.75)$ & $4.25(0.72)$ & $0.45^{*}$ \\
Wortspanne, 3-silbig & $3.66(0.53)$ & $3.77(0.52)$ & $3.56(0.51)$ & $0.40^{*}$ \\
Kunstwortnachsprechen & $19.49(3.20)$ & $20.41(2.64)$ & $18.66(3.44)$ & $0.58^{*}$ \\
Corsi-Block-Aufgabe & $5.13(1.05)$ & $5.38(1.01)$ & $4.90(1.03)$ & $0.47^{*}$ \\
Matrix-Aufgabe & $5.38(1.44)$ & $5.64(1.44)$ & $5.14(1.41)$ & $0.35^{*}$ \\
Wortspanne rückwärts & $3.58(0.60)$ & $3.73(0.62)$ & $3.44(0.53)$ & $0.51^{*}$ \\
Ziffernspanne rückwärts & $3.75(0.75)$ & $3.98(0.74)$ & $3.53(0.70)$ & $0.63^{*}$ \\
Zählspanne & $3.85(0.96)$ & $4.13(0.98)$ & $3.60(0.88)$ & $0.57^{*}$ \\
\hline
\end{tabular}

Anmerkungen. ${ }^{*} p<.01$; Cohens $d$ bezieht sich auf den Vergleich zwischen der Gruppe mit diagnostizierter Lernstörung und der Gruppe ohne Lernstörung.

Beide Versuchsgruppen unterscheiden sich in allen erhobenen Maßen des Arbeitsgedächtnisses. Die mit diesen Unterschieden verbundenen Effektgrößen bewegen sich im Bereich von $1 / 3$ bis zu 2/3 Standardabweichungen. Bedingt durch den höheren Anteil an Jungen in der Gruppe der Kinder mit diagnostizierter Lernstörung unterscheiden sich die Versuchsgruppen in der Variablen Geschlecht $(t(244)=3.06, p<.01, d=0.39)$. Bedeutsame Unterschiede im mittleren Alter finden sich hingegen nicht $(t(244)=0.04)$. 
Tabelle 4.1.2 zeigt getrennt für die beiden Versuchsgruppen die Korrelationsmatrix für die eingesetzten Arbeitsgedächtnistests.

Tabelle 4.1.2 Korrelationsmatrix

\begin{tabular}{|c|c|c|c|c|c|c|c|c|c|}
\hline Arbeitsgedächtnistest & 1 & 2 & 3 & 4 & 5 & 6 & 7 & 8 & 9 \\
\hline 1. Wortspanne, 1-silbig & -- & $.58 *$ & $.37 *$ & $.66^{*}$ & .11 & .15 & $.44^{*}$ & $.27 *$ & $.49^{*}$ \\
\hline 2. Wortspanne, 3-silbig & $.57 *$ & -- & $.28^{*}$ & $.61^{*}$ & .13 & .19 & $.34 *$ & $.23^{*}$ & $.36^{*}$ \\
\hline 3. Kunstwortnachsprechen & $.36^{*}$ & $.37 *$ & -- & $.38^{*}$ & -.06 & -.02 & $.23 *$ & $.33^{*}$ & $.24 *$ \\
\hline 4. Ziffernspanne & $.58 *$ & $.60^{*}$ & $.43^{*}$ & -- & .19 & .17 & $.44 *$ & $.37 *$ & $.51 *$ \\
\hline 5. Corsi-Block-Aufgabe & .19 & .15 & .17 & .22 & -- & $.47 *$ & .19 & $.44^{*}$ & $.31^{*}$ \\
\hline 6. Matrix-Aufgabe & $.24^{*}$ & .17 & $.28^{*}$ & $.35^{*}$ & $.49 *$ & -- & $.36^{*}$ & $.41^{*}$ & .21 \\
\hline 7. Wortspanne rückwärts & $.51^{*}$ & $.48^{*}$ & $.27 *$ & $.49 *$ & .20 & $.23 *$ & -- & $.47^{*}$ & $.49 *$ \\
\hline 8. Ziffernspanne rückwärts & $.45^{*}$ & $.39 *$ & $.35^{*}$ & $.51^{*}$ & .21 & .21 & $.53 *$ & -- & $.38^{*}$ \\
\hline 9. Zählspanne & $.43^{*}$ & $.44^{*}$ & $.29 *$ & $.45^{*}$ & .14 & .15 & $.39 *$ & $.46^{*}$ & -- \\
\hline
\end{tabular}

Anmerkungen. ${ }^{*} p<.01$; oberhalb der Diagonalen: Gruppe mit diagnostizierter Lernstörung; unterhalb der Diagonalen: Gruppe ohne Lernstörung.

Bezogen auf das hier postulierte mehrgliedrige Arbeitsgedächtnissystem zeigen sich hohe Interkorrelationen zwischen den Arbeitsgedächtnismaßen der jeweiligen Systemkomponente. Die der phonologischen Schleife zugewiesenen Maße korrelieren für beide Gruppen am höchsten mit der Ziffernspanne. Nominal etwas geringer fallen hier die Zusammenhänge mit dem Kunstwortnachsprechen aus. Ein hoher intrastruktureller Zusammenhang findet sich auch für die beiden Arbeitsgedächtnismaße des visuell-räumlichen Notizblocks, die in beiden Gruppen miteinander am höchsten korrelieren. Auch die Arbeitsgedächtnismaße der zentralexekutiven Komponente zeigen in beiden Gruppen hohe Interkorrelationen, modalitätsbedingt aber auch hohe Zusammenhänge zu Maßen der phonologischen Schleife. 
Ein Vergleich der Koeffizienten zwischen beiden Gruppen mittels KorrelationsDifferenzen-Test weist unter Berücksichtigung eines nach Bonferroni adjustierten AlphaFehlers ( $\alpha=.05 / 36$ Einzeltests) auf keine systematischen Unterschiede hin.

\section{Explorative und konfirmatorische Faktorenanalysen}

Zur Überprüfung der Beziehung zwischen den hier erhobenen Arbeitsgedächtnisvariablen und der theoretisch postulierten Struktur des Arbeitsgedächtnisses sowie deren Unabhängigkeit vom Status einer Lernstörung wurden konfirmatorische Faktorenanalysen mittels LISREL (Jöreskog \& Sörbom, 2005) berechnet.

Tabelle 4.1.3 beinhaltet die auf Kovarianzmatrizen beruhenden Schätzungen für die Koeffizienten (basierend auf Maximum Likelihood Parameterschätzungen) sowie die Anpassungsgüte der Modelle. Für die Gesamtgruppe zeigt das dreifaktorielle Arbeitsgedächtnismodell eine sehr gute Anpassung an die empirische Kovarianzmatrix $\left(\chi^{2}=\right.$ 31.75, $d f=24, p=.13)$. Zur Prüfung der Fragestellung, ob das so identifizierte Arbeitsgedächtnismodell unabhängig vom Vorliegen einer Lernstörung anwendbar ist, wurden simultane Modellschätzungen mit unterschiedlichen Restriktionen vorgenommen. Tabelle 4.1.3 zeigt die Parameterschätzungen und Fitindices des multiplen Gruppenvergleichs eines voll restringierten Modells (gleiche Struktur, gleiche Faktorkorrelationen, gleiche Faktorladungen, gleiche Messfehler für beide Gruppen). Auch für dieses Modell zeigt sich eine gute Anpassung $\left(\chi^{2}=79.14, d f=69, p=.19\right)$. Durch die sukzessive Lockerung der Restriktionen können hierarchische Modelltests durchgeführt und die Verbesserung der Modellanpassung mittels $\chi^{2}$-Differenzen-Test auf statistische Bedeutsamkeit hin geprüft werden. Der multiple Gruppenvergleich eines unrestringierten Modells (gleiche Struktur für beide Gruppen) zeigt mit $\chi^{2}=49.20(d f=48, p=.42, R M S E A=.01)$ ebenfalls eine gute Modellanpassung. Ein Vergleich beider Modellschätzungen zeigt, dass die gruppenspezifisch freie Schätzung der Parameter zu keiner signifikant besseren Modellanpassung führt (partielles $\chi^{2}=29.94, d f=21, p=.09$ ). Für beide Gruppen kann daher von einer analogen Modellstruktur mit vergleichbaren Faktorladungen und -korrelationen sowie Messfehlern ausgegangen werden. 
Tabelle 4.1.3 Parameter und Fitmaße der konfirmatorischer Faktorenanalysen

\begin{tabular}{|c|c|c|}
\hline & Modell der Gesamtgruppe & $\begin{array}{l}\text { Modell mit getrennten } \\
\text { Lernstörungsgruppen }\end{array}$ \\
\hline \multicolumn{3}{|l|}{ Faktorladungen } \\
\hline PS --> Wortspanne, 1-silbig & .78 & .77 \\
\hline PS --> Wortspanne, 3-silbig & .73 & .72 \\
\hline PS --> Kunstwortnachsprechen & .52 & .47 \\
\hline PS --> Ziffernspanne & .84 & .83 \\
\hline VRN --> Corsi-Block-Spanne & .70 & .66 \\
\hline VRN --> Matrix-Spanne & .72 & .72 \\
\hline ZE --> Wortspanne rückwärts & .72 & .70 \\
\hline ZE --> Ziffernspanne rückwärts & .70 & .67 \\
\hline ZE --> Zählspanne & .68 & .65 \\
\hline \multicolumn{3}{|l|}{ Faktorinterkorrelationen } \\
\hline PS <--> VRN & .41 & .35 \\
\hline $\mathrm{ZE}<-->\mathrm{PS}$ & .84 & .82 \\
\hline ZE $<-->$ VRN & .59 & .55 \\
\hline \multicolumn{3}{|c|}{ Messfehler der manifesten Variablen } \\
\hline --> Wortspanne, 1-silbig & .39 & .40 \\
\hline --> Wortspanne, 3-silbig & .46 & .48 \\
\hline --> Kunstwortnachsprechen & .73 & .78 \\
\hline --> Ziffernspanne & .29 & .32 \\
\hline --> Corsi-Block-Spanne & .51 & .56 \\
\hline --> Matrix-Spanne & .49 & .48 \\
\hline --> Wortspanne rückwärts & .48 & .50 \\
\hline --> Ziffernspanne rückwärts & .50 & .55 \\
\hline --> Zählspanne & .54 & .58 \\
\hline \multicolumn{3}{|l|}{ Fitmaße $^{2}$} \\
\hline$\chi^{2} / d f$ & 1.32 & 1.15 \\
\hline RMSEA & .04 & .03 \\
\hline NNFI & .99 & .99 \\
\hline CFI & .99 & .99 \\
\hline krit. $N$ & 323.66 & 311.04 \\
\hline
\end{tabular}

Anmerkungen. 1) Restriktionen: gleiche Struktur, gleiche Faktorkorrelationen, gleiche Faktorladungen, gleiche Messfehler; PS: phonologische Schleife; ZE: zentrale Exekutive; VRN: visuell-räumlicher Notizblock. 2)

Konventionen für einen ,guten Modellfit“: RMSEA $\leq 05$, NNFI und CFI jeweils $\geq .97$ (vgl. Schermelleh-Engel, Moosbrugger \& Müller, 2003) 
Da Jarvis und Gathercole (2003; vgl. auch Shah \& Miyake, 1996) in der Diskussion zur Struktur des Arbeitsgedächtnisses ein zweigliedriges Modell vorgeschlagen haben, führten wir darüber hinaus eine explorative Faktorenanalyse durch. Diese führte zu einer 2Faktorlösung mit einer Varianzaufklärung von 59.79\%. Die rotierte Komponentenmatrix verweist auf einen visuell-räumlichen Faktor (Corsi-Block-Aufgabe, Matrix-Aufgabe) sowie einen verbalen Faktor (alle anderen Arbeitsgedächtnistests), was gut zu den Überlegungen von Jarvis und Gathercole (2003) passt. Lediglich die Ziffernspanne rückwärts zeigt hohe Ladungen auf beiden Faktoren. Hierarchische Modelltests ergaben jedoch, dass ein entsprechendes konfirmatorisches Modell zu dieser Lösung $\left(\chi^{2}=53.49, d f=25, p<.01\right.$, $R M S E A=.07, N N F I=.97, C F I=.98)$ dem dreifaktoriellen Arbeitsgedächtnisstrukturmodell unterlegen ist (partielles $\chi^{2}=21.74, d f=1, p<.01$ ). Betrachtet man den Overall-Modelfit (RMSEA) beider Modelle, so genügt das zweifaktorielle Modell nicht mehr dem Kriterium einer „guten Modellanpassung“ mit einem RMSEA $\leq .05$ (vgl. Schermelleh-Engel, Moosbrugger \& Müller, 2003). Dies gilt auch, wenn die 2-Faktorlösung strikt modalitätsspezifisch gestaltet wird. Dieses Modell $\left(\chi^{2}=71.81, d f=26, p<.01, R M S E A=.08\right.$, $N N F I=.96, C F I=.97)$ zeigt ebenfalls eine eher schlechtere Anpassung an die Daten und ist dem dreigliedrigen Arbeitsgedächtnisstrukturmodell nicht überlegen (partielles $\chi^{2}=40.06, d f$ $=2, p<.01)$. Somit führt das zweigliedrige Modell weder inhaltlich-theoretisch noch statistisch zu einer besseren Anpassung als das dreigliedrige Modell (vgl. Alloway et al., 2006).

\section{Diskussion}

Das Ziel der vorliegenden Studie war es zu prüfen, ob die Struktur des Arbeitsgedächtnisses im Altersbereich zwischen 6 und 10 Jahren bei Kindern mit vs. ohne Lernstörungen äquivalent ist (Struktur-Äquivalenz-Hypothese). Dazu wurde mit insgesamt 245 Kindern dieser Altersspanne eine ausführliche Testbatterie zur Erfassung der verschiedenen Funktionsbereiche des Arbeitsgedächtnisses durchgeführt. Etwa die Hälfte dieser Kinder hatte eine Lernstörungsdiagnose, die übrigen nicht. Für beide Gruppen ließ sich ohne Einschränkung die identische dreigliedrige Arbeitsgedächtnisstruktur nach Baddeley (1986, 2006) finden. Konfirmatorische Analysen zum Basismodell für die Gesamtgruppe belegen auch nach Auspartialisierung des Alters $\left(\chi^{2}=32.78, d f=24, p=.11, R M S E A=.04, N N F I=\right.$ $.99, C F I=.99)$ oder des Geschlechts $\left(\chi^{2}=32.58, d f=24, p=.11, R M S E A=.04, N N F I=.99\right.$, 
CFI = .99) die gute Anpassung des Modells an die Daten (Basis in diesen Fällen: Korrelationsmatrizen).

Zusammenfassend lässt sich festhalten, dass das dreigliedrige Modell des Arbeitsgedächtnisses sich somit insgesamt auch für Kinder mit diagnostizierter Lernstörung sehr gut bewährt hat. Dies eröffnet die optimistische Sicht, dass dieses Modell auch als Grundlage für die Konstruktion und Anwendung von Arbeitsgedächtnis-Testbatterien zur Differentialdiagnose der Funktionsdefizite im Arbeitsgedächtnis bei Kindern mit Lernstörungen geeignet ist.

\section{Zusammenfassung}

245 Kinder der ersten bis vierten Klassenstufe bearbeiteten eine ausführliche Testbatterie zur Erfassung der Funktionstüchtigkeit bei phonologischen, visuell-räumlichen und zentralexekutiven Arbeitsgedächtnisanforderungen. Etwa die Hälfte dieser Kinder leidern unter einer diagnostizierten Lernstörung (ICD-10). Mit Hilfe statistischer Dimensions- und Strukturanalysen konnte gezeigt werden, dass die der verwendeten Arbeitsgedächtnistestbatterie zugrunde liegende Vorstellung einer drei vergleichsweise unabhängigen Funktionsstruktur des Arbeitsgedächtnisses in gleicher Weise Gültigkeit hat für Kinder mit vs. ohne Lernstörung.

Somit liefert diese Studie eine empirische Bestätigung der Struktur-ÄuqivalenzHypothese und bildet die Grundlage dafür, in den nächsten Schritten (Studie 2 und Studie 3) die Arbeitsgedächtnisleistungen bei Kindern mit spezifischen Lernstörungen detailliert zu analysieren, um spezifische Defizitmuster ausfindig zu machen und Leistungseinbußen nicht auf Strukturdefizite sondern auf Funktionsdefizite zurückzuführen. 


\subsection{Studie 2: Welche Funktionsbereiche des Arbeitsgedächtnisses sind bei Kindern mit Lese-Rechtschreib- und/oder Rechenstörung beeinträchtig? ${ }^{2}$}

Children with learning disabilities like dyslexia or dyscalculia tend to experience significant difficulties in acquiring the core skills of reading, writing, and arithmetic from their very first days at school. Even when the instruction they receive is attuned to their special learning needs, these difficulties tend to persist or increase over the school career. Dyslexia is characterized by a specific and significant impairment in the development of reading skills (often accompanied by poor spelling); dyscalculia by a specific impairment in the acquisition of mathematical skills. There are numerous approaches to the definition and diagnosis of these learning disabilities. Internationally recognized criteria for their diagnosis are specified in the conventional classification systems: the International Classification of Diseases (ICD10) published by the World Health Organization (WHO, 2005), and the Diagnostic and Statistical Manual of Mental Diseases (DSM-IV-TR) published by the American Psychiatric Association (2000). According to these systems, learning disorders are present when individuals' abilities in the domains of reading, spelling, or arithmetic are substantially below their expected potential given their age, general intelligence, and education. In other words, there must be a considerable discrepancy between general intellectual ability and academic achievement. Although there is an old and enduring controversy concerning the reliability of the discrepancy criterion (Francis et al., 2005; Siegel, 1989; Stanovich, 2005) the WHO and APA still adhere to these selection criteria. Cutoff points for the size of this critical discrepancy vary between 1 and 2 standard deviations of the psychometric norm distributions. Recent prevalence studies applying the ICD-10 criteria indicate that between $4 \%$ and $7 \%$ of children are affected by dyslexia (Lewis, Hitch, \& Walker, 1994; Miles, Haslum, \& Wheeler, 1998; Rutter et al., 2004; Shaywitz, Escobar, Shaywitz, Fletcher, \& Makuch, 1992). Hardly less extended prevalence rates (3-6\%) are reported for dyscalculia (Fuchs et al., 2005; Lewis et al., 1994; Mazzocco \& Myers, 2003).

Despite increasing research interest in learning disabilities, consensus has not yet been reached on the specific cognitive deficits that underlie different learning disorders. For a long time dyslexia was considered to be caused by visual deficits. While currently deficits in phonological information processing are demonstrated to be responsible (Vellutino, Fletcher, Snowling, \& Scanlon, 2004). Causation of dyscalculia is much less clear. Deficiencies in the

\footnotetext{
2 Dieses Kapitel basiert auf einer Publikation, die im Journal of Learning Disabilities erscheinen wird (Schuchardt, K., Mähler, C., \& Hasselhorn, M. (in press). Working memory deficits in children with specific learning disorders.).
} 
memory of basic facts, immature strategies and a less developed number sense are taken into consideration (Geary, 2004).

In recent years, there has been particular interest in deficits in memory, especially working memory, which is responsible for the processing and short-term storage of information. Various models of working memory have been developed, and the model provided by Baddeley (1986) has proved a particularly useful theoretical tool in numerous studies on learning disabilities. The model distinguished between three components of working memory: the modality-free central executive is a kind of supervisory system that serves to control and regulate the cognitive processes occurring in its two limited-capacity slave systems, the phonological loop and the visual-spatial sketchpad. Further functions of the central executive that have since been identified by Baddeley (1996) include coordinating the slave systems, focusing and switching attention, and retrieving representations from longterm memory. The two slave systems perform modality-specific operations. Verbal and auditory information are stored temporarily and processed in the phonological loop. Two components of the phonological loop are distinguished: the phonological store and the subvocal rehearsal process. The visual-spatial sketchpad is concerned with remembering and processing visual and spatial information; it comprises a visual cache for static visual information and an inner scribe for dynamic spatial information (Logie, 1995; Pickering, Gathercole, Hall, \& Lloyd, 2001).

Research has provided numerous indications that specific learning disabilities are associated with impairments in working memory (Alloway \& Gathercole, 2006; Pickering, 2006a). It is undisputed that children with specific reading disabilities have deficits in phonological processing and storage (Pickering, 2006b; Swanson, 2006; Vellutino et al., 2004), and there is evidence to suggest that they also experience deficits in central executive functioning (Landerl et al., 2004; Palmer, 2000; Siegel \& Ryan, 1989; Swanson, 1993, 1999). There are relatively few reports of impairments in the visual-spatial working memory of reading disabled children, however (Eden \& Stein, 1995; Howes, Bigler, Burlingame, \& Lawson, 2003; Kibby, Marks, Morgan, \& Long, 2004; O’Shaughnessy \& Swanson, 1998; Pickering, 2006b).

Empirical findings on children with specific arithmetic learning disabilities are also available for all three domains of working memory (Passolunghi, 2006). There is much evidence to suggest that not all components of working memory show the same kinds of deficits. The central executive seems to be particularly impaired (Geary, Brown, \& 
Samaranayake, 1991; Geary Hamson, \& Hoard, 2000; Geary, Hoard, \& Hamson, 1999; Hitch \& McAuley, 1991; McLean \& Hitch, 1999; Passolunghi \& Siegel, 2001; Siegel \& Ryan, 1989; Swanson, 1993; Swanson \& Sachse-Lee, 2001; Wilson \& Swanson, 2001), but findings on the phonological loop are contradictory. Whereas Geary et al. (1991), Hitch, and McAuley (1991), and Swanson and Sachse-Lee (2001) found children with specific arithmetic learning difficulties to show deficits in phonological working memory, no evidence of such impairment was found in the studies by Bull, Johnston, and Roy (1999), Geary et al. (1999, 2000), McLean \& Hitch (1999), or Landerl, Bevan, and Butterworth (2004). Thus, deficits in the phonological loop may not be a defining characteristic of children with arithmetic learning disabilities (Passolunghi, 2006). Recent studies (D’Amico \& Guarnera, 2005; Reuhkala, 2001; McLean \& Hitch, 1999; van der Sluis, van der Leij, \& de Jong, 2005; see also Passolunghi, 2006) have also reported visual-spatial deficits in children with specific arithmetic disability. However, Bull et al. (1999) and Geary et al. (2000) report arithmetic disabled children and their normally achieving peers to have comparable outcomes on measures of visual-spatial memory.

In sum, research findings on specific impairments of working memory are rather heterogeneous. Potential reasons for these mixed results include the limited comparability of the samples studied, and differences in the selection criteria applied and in the instruments used. For example, classifications of learning disabled students differ across studies, with performance below the 25 th or 35 th percentile on a standardized test often being taken as the decisive criterion. Only few studies have taken into account of intellectual ability or have specified a critical discrepancy between ability and achievement. It thus remains unclear whether the findings reported in previous research also apply to children with learning disorders as defined above. Additionally, there are notable differences in the procedures used to diagnose learning disorders. Moreover, the various functions of working memory have as yet been operationalized by only a few, very different tasks, thus precluding differentiated analysis of individual components of working memory.

One reason for the heterogeneity of results regarding the precise nature of working memory deficits in children with specific learning disabilities might be the high rate of comorbidity of different learning disabilities. Numerous epidemiological studies have shown a comorbidity between dyscalculia and dyslexia (Badian, 1983; Gross-Tsur, Manor, \& Shalev, 1996; Lewis, Hitch, \& Walker, 1994; Ramaa \& Gowramma, 2002; cf. Hasselhorn \& Schuchardt, 2006). For example, Lewis et al. (1994) found that $2.3 \%$ of the children in their study had difficulties with both reading and arithmetic. Because most previous studies have 
examined the two disorders separately, little is yet known about the deficits in cognitive functioning present in children with mixed disorders of scholastic skills. Broad deficits in cognitive functioning, and particularly in memory, are assumed. Studies comparing children with specific arithmetic or reading disabilities, on the one hand, with children with combined learning disabilities, on the other, show that the latter group has particularly serious deficits in working memory functions (Geary et al., 1999, 2000; Siegel \& Ryan 1989; van der Sluis et al., 2005). A recent study has analysed children classified as learning disabled in arithmetic, reading/spelling, or both, but who did not show a notable discrepancy between intellectual ability and achievement (Schuchardt, Kunze, Grube, \& Hasselhorn, 2006). No evidence was found of an independent, characteristic pattern of deficits in working memory in the latter group. Similar findings were reported by van der Sluis et al. (2005). However, it remains unclear whether the same applies to children with clinically relevant learning difficulties in both domains.

The aim of the present study was to investigate the functioning of the three subcomponents of working memory in children who meet the ICD-10 criteria for clinically relevant learning disorders. Assessing children with specific learning disorders, i.e., dyslexia or dyscalculia, and children with mixed learning disabilities within a single study design makes it possible to compare the specific deficits in working memory underlying each disorder. Furthermore, using a comprehensive battery of working memory measures including various indicators of the three subcomponents makes it possible to explore whether there are specific deficits in individual components of working memory.

Method

\section{Design}

We used two-factor mixed design: (1) presence or absence of dyscalculia and (2) presence or absence of dyslexia. To this end, we identified three groups of learning disabled children based on the ICD-10 criteria for specific developmental disorders of scholastic skills: children with specific disorders of arithmetical skills (AD), children with specific reading disorders (RD), and children with mixed disorders of scholastic skills (AD+RD), and formed a control group (C) of normally achieving children matched for chronological age. 


\section{Participants}

Ninety-seven $2^{\text {nd }}$ to $4^{\text {th }}$ grade students (age $7-10$ years) participated in the study. All children were screened with standardized tests of intellectual ability, spelling, reading, and arithmetic. We used the full IQ scale from the German version of the Kaufman Assessment Battery for Children (K-ABC, Melchers \& Preuß, 2001) to assess general intelligence. Mathematical skills were assessed using standardized German mathematical achievement tests for $2^{\text {nd }}, 3^{\text {rd }}$, and $4^{\text {th }}$ graders (DEMAT 2+, Krajewski, Liehm, \& Schneider, 2004; DEMAT 3+, Roick, Gölitz, \& Hasselhorn, 2004; DEMAT 4, Gölitz, Roick, \& Hasselhorn, 2006). These multicomponent tests include computation problems, word problems, and geometry problems. Spelling abilities were assessed by the Weingartener spelling tests for $2^{\text {nd }}$ and $3^{\text {rd }}$ graders (WRT 2+, Birkel, 1994a; WRT 3+, Birkel, 1994b) and the Westermann spelling test for $4^{\text {th }}$ graders (WRT 4/5, Rathenow, 1980). In both of these standardized German achievement tests, children insert dictated words into given sentences. Reading speed abilities were classified on the basis of scores on two subtests of the Salzburg reading test (SLT; Landerl, Wimmer, \& Moser, 1997): the word reading subtest "Textlesen" (short or long version depending on grade level) and the nonword reading subtest "Wortunähnliche Pseudowörter."

Only native German-speaking children were included in the study sample. The operational criteria for the learning disability subgroups were as follows: (a) IQ $\geq 80$, (b) below-average reading, spelling, and/or arithmetic scores $(\mathrm{T}<40$ [ i.e., T-scores: mean of 50 and SD of 10] or percentile $<16$ ), and (c) a critical discrepancy of 1.2 standard deviations between IQ and overall performance on the standardized tests of school achievement. We defined 17 children with T-scores lower than 40 (percentile $<16$ ) in mathematics and Tscores higher than 40 (percentile $\geq 16$ ) in reading and spelling as belonging to the AD group, and 30 children with $\mathrm{T}$-scores lower than 40 (percentile $<16$ ) in reading and spelling but higher than 40 (percentile $\geq 16$ ) in mathematics as belonging to the RD group. Children with T-scores lower than 40 (percentile $<16$ ) on both the reading and arithmetic tests were classified as belonging to the $\mathrm{AD}+\mathrm{RD}$ group $(\underline{\mathrm{n}}=20)$. The control group $(\underline{\mathrm{n}}=30)$ showed average performance (percentile $\geq 16$ ) on all achievement tests.

We recruited the three learning disability groups from the counseling center for children with learning disabilities at our department. Control group children were $2^{\text {nd }}$ and $4^{\text {th }}$ graders from a public elementary school. None of the children in the study attended special schools. 
Table 4.2.1 Sex distribution and Means (Standard Deviations) and [ Ranges] of Age, K-ABC IQ, WRT Spelling T-Scores, SLT Word and Nonword Reading T-Scores, and DEMAT Mathematics T-Scores by Subgroup

\begin{tabular}{lcccc}
\hline & AD & RD & AD+RD & C \\
& $(N=17)$ & $(N=30)$ & $(N=20)$ & $(N=30)$ \\
\hline Sex (m/f) & $5 / 12$ & $18 / 12$ & $8 / 12$ & $15 / 15$ \\
Age (months) & $103.41(9.11)$ & $108.57(10.46)$ & $103.70(11.19)$ & $108.77(9.53)$ \\
& {$[92-124]$} & {$[93-128]$} & {$[87-132]$} & {$[90-126]$} \\
IQ & $93.47(8.83)$ & $99.67(7.31)[85-$ & $92.80(7.63)$ & $100.53(6.41)$ \\
Spelling & {$[80-109]$} & $116]$ & {$[80-105]$} & {$[89-110]$} \\
& $46.81(8.55)$ & $33.13(4.83)$ & $32.10(6.63)$ & $50.97(6.41)$ \\
Word reading & {$[41-61]$} & {$[21-39]$} & {$[20-39]$} & {$[41-61]$} \\
& $46.55(10.16)^{*}$ & $32.62(7.02)$ & $31.05(8.08)$ & $52.17(8.42)$ \\
Nonword reading & {$[40-56]$} & {$[23-39]$} & {$[21-39]$} & {$[41-63]$} \\
& $48.00(11.37)^{*}$ & $33.48(8.06)$ & $35.21(11.38)$ & $49.04(8.71)$ \\
Mathematics & {$[41-61]$} & {$[20-39]$} & {$[23-39]$} & {$[41-59]$} \\
& $30.35(4.46)$ & $48.00(8.19)$ & $30.90(5.51)$ & $50.43(5.59)$
\end{tabular}

Note. $\mathrm{AD}=$ children with specific disorders of arithmetical skills; $\mathrm{RD}=$ children with specific reading disorders; $\mathrm{AD}+\mathrm{RD}$ children with mixed disorders of scholastic skills; $\mathrm{C}=$ normally achieving control children matched for chronological age. ${ }^{*}$ SLT data were missing for six participants.

Table 4.2.1 summarizes the four groups' descriptive statistics. On average, the AD and $\mathrm{AD}+\mathrm{RD}$ groups performed significantly lower on the mathematics abilities test than did the $\mathrm{C}$ and $\mathrm{RD}$ groups. At the same time, the $\mathrm{RD}$ and $\mathrm{AD}+\mathrm{RD}$ groups scored significantly lower on spelling and reading tests than did the $\mathrm{C}$ and $\mathrm{AD}$ groups. Inspection of gender distribution patterns across learning disability groups showed that more $\mathrm{AD}(70 \%)$ and $\mathrm{AD}+\mathrm{RD}(60 \%)$ children were female, whereas more RD children were male $(60 \%)$. There were no differences in gender distribution between children with and without low math achievement $\left(\chi^{2}(1)=2.87\right.$, $p>.05)$ and children with and without low achievement scores in reading and spelling $\left(\chi^{2}(1)\right.$ $=0.53, p>.05)$. Analysis of variance revealed that the four experimental groups did not differ significantly in terms of age, $F(3,93)=1.94, \eta^{\uparrow}=.06, M S E=102.36, p>.05$. However, the $\mathrm{AD}$ and $\mathrm{AD}+\mathrm{RD}$ groups were on average 5 months younger than the $\mathrm{RD}$ and $\mathrm{C}$ groups. The groups did differ significantly in terms of intelligence $F(3,93)=6.91, \eta^{\uparrow}=.18, M S E=54.75, p$ 
$<.05$. Post hoc comparisons showed that there was no difference in intelligence between the $\mathrm{C}$ and the $\mathrm{RD}$ group or between the $\mathrm{AD}$ and the $\mathrm{AD}+\mathrm{RD}$ groups, but that the $\mathrm{AD}$ and $\mathrm{AD}+\mathrm{RD}$ groups scored significantly lower on intelligence than the other two groups. We therefore included age and general intelligence as covariates in all subsequent analyses.

\section{Working memory assessment}

Working memory was assessed by a battery of 16 tasks: 7 phonological tasks (memory spans for digits, one-syllable and three-syllable words, one-syllable and three-syllable nonwords, and images; nonword repetition), 5 visual-spatial tasks (memory span for locations, matrix span simple and complex, corsi-block simple and complex), 4 central executive tasks (double span, backward spans for one-syllable words and digits, counting span). A detailed description of all tasks follows below.

Phonological loop. The digit span is the conventional measure used to assess phonological short-term capacity. A series of one to nine digits was presented acoustically at a rate of one digit per second, starting with two and continuing up to a maximum of eight digits. Participants had to repeat the digits immediately in the presented order. The one-syllable and three-syllable word span tasks and the one-syllable and three-syllable nonword span tasks were presented in the same manner as in the digit span measure. In the one-syllable and threesyllable word span tasks familiar German nouns (e.g., Stern=star, Fisch=fish, Erdbeere=strawberry, Briefkasten=letterbox) were used; the one-syllable and three-syllable nonword span tasks are word-like nonwords (e.g., fen, sim, bestrugeln, reseubelt). In the images span task, participants were presented a series of pictures of easily recognizable objects (e.g., sun, umbrella, door, car) on a computer screen, and were asked to recall them in the order of presentation. The German nonword repetition task administered was developed by Hasselhorn and Körner (1997). Children had to repeat 24 word-like nonwords of 2, 3, or 4 syllables immediately after their presentation. Nonwords of different lengths were presented auditorily in random order. The number of correctly repeated nonwords was taken as the score for this task.

Visual-spatial sketchpad. In the location span task, children were shown a series of green dots at different locations on a $3 \times 3$ matrix and asked to recall these locations in the correct order. Corsi-block tasks were used to assess the dynamic spatial component of visual- 
spatial memory. Nine red blocks are nailed in random positions on a gray board $(23 \times 27.5$ $\mathrm{cm})$. The experimenter taps a sequence of blocks at the rate of one per second. The child then attempts to reproduce the sequence of taps in the correct order. We used two variations of the corsi-block task: simple sequences involving short distances between blocks without path crossings, and complex sequences involving long distances between blocks with path crossings. A matrix span task was incorporated in the battery to measure the static component of visual-spatial working memory. This task assesses memory for random visual-spatial patterns of increasing complexity. Patterns of white and black boxes in a 4x4 matrix were presented on the computer, beginning with two black boxes and continuing up to a maximum of eight black boxes. Immediately after presentation, children were asked to reproduce the pattern in an empty matrix. Two variations of this task were also implemented: a simple matrix span with the black boxes arranged in simple patterns, and a complex matrix span with the black boxes located at some distance from one another.

Central executive. The same items and procedures were used for the backward digit and word span tasks as for the forward spans, the only difference being that participants were required to recall the sequences of items in reverse order. Additionally, a double span task was implemented to assess the children's ability to coordinate the functioning of the phonological loop and the visual-spatial sketchpad. The same pictures as in the images span task were presented, but this time in different locations on a $3 \times 3$ matrix. Children had to recall simultaneously the pictures by verbally recoding the semantic content (phonological demand) and their location (visual-spatial demand) in the order of presentation. Thus, this task is properly viewed as a central executive task due to its coordinative requirements. The complex counting span task, a measure of storage and processing efficiency, was based on a task designed by Case, Kurland, and Goldberg (1982). A series of yellow circles (target items) and squares (distractor items) was presented in a random, computer-generated pattern. Children were instructed to count the number of circles. Subsequently, another map was presented and children again had to count the number of circles. Finally, the experimenter asked the child to recall the number of circles counted on each map. The number of maps presented per sequence was steadily increased up to a maximum of eight.

Stop criterion. We used the same stop criterion for all span tasks. The length of the sequences presented was increased gradually, beginning with a minimum of two, and 
increasing to a maximum of eight items. There were four trials at each sequence length. If an error was made, the child was given a second attempt at an item of the same length. If a child succeeded on two successive trials of the same length, the task continued with the next span length. If a child failed on two successive trials of the same length, he or she was not presented with any further sequences of the same length, but with a sequence one item shorter. The dependent measure for all span tasks was the longest sequence of items repeated in correct order. Children were credited an extra fourth point if they repeated a further sequence of the same length correctly (e.g., a score of 5.25 was awarded if two of four 5-item sequences were recalled correctly, 5.5 if three of four sequences, and 5.75 if all four sequences were recalled correctly).

Procedure. Children with learning disabilities were administered standardized tests of spelling, reading, and arithmetic, intelligence, and working memory individually in two separate sessions. The DEMAT and WRT measures were administered to control group children in classroom learning groups. All other tests were administered individually within a period of 3 weeks. Except for the corsi-block task, all working memory tasks were administered by computer. The order of presentation of the working memory tasks was the same for all children (images span, location span, double span, one-syllable word span, threesyllable word span, corsi-block simple, corsi-block complex, nonword repetition, backward word span, backward digit span, counting span, digit span, matrix span simple, matrix span complex, one-syllable nonword span, three-syllable nonword span).

\section{Results}

For all tests the alpha level was set $p=.05$. Table 4.2 .2 presents means and standard deviations for all working memory measures by the four groups. Scores on the three components of working memory tasks were entered in multivariate analyses of variance with presence/absence of dyscalculia and dyslexia as fixed factors. IQ and chronological age were included as covariates in all subsequent analyses. 
Table 4.2.2 Means (Standard Deviations) for Working Memory Measures by Subgroup

\begin{tabular}{|c|c|c|c|c|}
\hline & $\mathrm{AD}$ & $\mathrm{RD}$ & $\mathrm{AD}+\mathrm{RD}$ & $\mathrm{C}$ \\
\hline \multicolumn{5}{|l|}{ Phonological loop } \\
\hline Digit span & $5.09(0.74)$ & $4.63(0.64)$ & $4.19(0.50)$ & $5.19(0.61)$ \\
\hline One-syllable word span & $4.47(0.67)$ & $4.28(0.74)$ & $3.85(0.58)$ & $4.62(0.66)$ \\
\hline Three-syllable word span & $3.67(0.47)$ & $3.64(0.47)$ & $3.24(0.46)$ & $3.86(0.43)$ \\
\hline One-syllable nonword span & $3.85(0.58)$ & $3.47(0.89)$ & $3.19(0.96)$ & $4.27(0.50)$ \\
\hline \multirow{2}{*}{$\begin{array}{l}\text { Three-syllable nonword span Images } \\
\text { span }\end{array}$} & $2.09(1.27)$ & $1.34(1.22)$ & $1.27(1.22)$ & $2.43(0.74)$ \\
\hline & $4.35(0.72)$ & $4.22(0.77)$ & $3.71(0.56)$ & $4.60(0.84)$ \\
\hline Nonword repetition & $19.76(2.11)$ & $17.47(3.13)$ & $17.85(4.66)$ & $20.93(1.78)$ \\
\hline \multicolumn{5}{|l|}{ Visual-spatial sketchpad } \\
\hline Location span & $4.60(0.75)$ & $5.26(1.00)$ & $4.36(0.66)$ & $4.98(0.80)$ \\
\hline Corsi-block simple & $5.43(0.98)$ & $5.68(1.34)$ & $4.94(1.10)$ & $5.78(1.36)$ \\
\hline Corsi-block complex & $3.90(0.95)$ & $4.80(0.85)$ & $3.87(1.24)$ & $4.98(1.08)$ \\
\hline Matrix span simple & $5.37(1.63)$ & $6.72(1.11)$ & $5.31(1.15)$ & $6.50(1.29)$ \\
\hline Matrix span complex & $3.00(1.25)$ & $4.45(1.63)$ & $3.31(1.08)$ & $4.72(1.52)$ \\
\hline \multicolumn{5}{|l|}{ Central executive } \\
\hline Backward digit span & $3.71(0.56)$ & $3.45(0.66)$ & $3.10(0.86)$ & $3.90(0.68)$ \\
\hline Backward words span & $3.46(0.57)$ & $3.58(0.56)$ & $3.19(0.31)$ & $3.82(0.63)$ \\
\hline Double span & $3.76(0.79)$ & $3.78(0.76)$ & $3.31(0.55)$ & $4.04(0.72)$ \\
\hline Counting span & $3.87(0.97)$ & $3.81(0.89)$ & $3.20(0.71)$ & $4.45(0.97)$ \\
\hline
\end{tabular}

Note. $\mathrm{AD}=$ children with specific disorders of arithmetical skills; $\mathrm{RD}=$ children with specific reading disorders; $\mathrm{AD}+\mathrm{RD}$ children with mixed disorders of scholastic skills; $\mathrm{C}=$ normally achieving control children matched for chronological age.

\section{Phonological loop}

The scores on the seven tasks assessing phonological loop functioning were entered into a MANCOVA. The multivariate main effect for dyslexia, $F(7,85)=6.91, \eta^{2}=.36, p<.001$, proved to be significant. The multivariate main effect for dyscalculia, $F(7,85)<1$ and the dyslexia by dyscalculia interaction were not significant, $F(7,85)<1$. Table 4.2 .3 presents all univariate tests of phonological working memory. For the dyslexia factor, univariate tests showed significant differences between groups on all phonological tasks. 
Table 4.2.3 Phonological Loop - Univariate Tests for the Factors Dyscalculia, Dyslexia, and the Dyscalculia * Dyslexia Interaction

\begin{tabular}{|c|c|c|c|}
\hline & $F$ & $p$ & $\eta^{2}$ \\
\hline \multicolumn{4}{|l|}{ Dyscalculia } \\
\hline Digit span & $<1$ & ns & - \\
\hline One-syllable word span & $<1$ & ns & - \\
\hline Three-syllable word span & 2.60 & ns & - \\
\hline One-syllable nonword span & 1.38 & ns & - \\
\hline \multirow{2}{*}{$\begin{array}{l}\text { Three-syllable nonword span } \\
\text { Images span }\end{array}$} & $<1$ & ns & - \\
\hline & $<1$ & ns & - \\
\hline Nonword repetition & $<1$ & ns & - \\
\hline \multicolumn{4}{|l|}{ Dyslexia } \\
\hline Digit span & 32.13 & .000 & .261 \\
\hline One-syllable word span & 11.36 & .001 & .111 \\
\hline Three-syllable word span & 12.27 & .001 & .119 \\
\hline One-syllable nonword span & 21.27 & .000 & .189 \\
\hline \multirow{2}{*}{$\begin{array}{l}\text { Three-syllable nonword span } \\
\text { Images span }\end{array}$} & 16.94 & .000 & .157 \\
\hline & 10.64 & .002 & .105 \\
\hline Nonword repetition & 17.65 & .000 & .162 \\
\hline \multicolumn{4}{|l|}{ Dyscalculia * Dyslexia } \\
\hline Digit span & 1.92 & ns & - \\
\hline One-syllable word span & 1.19 & ns & - \\
\hline Three-syllable word span & 1.58 & ns & - \\
\hline One-syllable nonword span & $<1$ & ns & - \\
\hline Three-syllable nonword span & $<1$ & ns & - \\
\hline Images span & $<1$ & ns & - \\
\hline Nonword repetition & 1.48 & ns & - \\
\hline
\end{tabular}

\section{Visual-spatial sketchpad}

The scores on the five tasks assessing visual-spatial sketchpad were entered into a multivariate analysis of variance (MANCOVA). The multivariate main effect for dyscalculia, $F(5,87)=4.31, \eta^{\uparrow}=.20, p<.01$, proved significant. In contrast, the multivariate main effect for dyslexia, $F(5,87)<1$, and the dyscalculia $x$ dyslexia interaction, $F(5,87)=1.44$, $\Upsilon^{2}=.08$, 
$p>.05$, were not significant. The univariate tests of visual-spatial sketchpad are presented in Table 4.2.4. For the dyscalculia factor, univariate tests of visual-spatial working memory tasks revealed significant differences between groups on all visual-spatial memory tasks with the exception of the corsi-block simple task.

Table 4.2.4 Visual-spatial sketchpad - Univariate Tests for the Factors Dyscalculia, Dyslexia, and the Dyscalculia * Dyslexia Interaction

\begin{tabular}{|c|c|c|c|}
\hline & $F$ & $p$ & $\eta^{2}$ \\
\hline \multicolumn{4}{|l|}{ Dyscalculia } \\
\hline Location span & 6.25 & .014 & .064 \\
\hline Corsi-block simple & 2.48 & ns & - \\
\hline Corsi-block complex & 13.06 & .000 & .125 \\
\hline Matrix span simple & 8.42 & .005 & .085 \\
\hline Matrix span complex & 12.96 & .001 & .125 \\
\hline \multicolumn{4}{|l|}{ Dyslexia } \\
\hline Location span & $<1$ & ns & - \\
\hline Corsi-block simple & 1.25 & ns & - \\
\hline Corsi-block complex & $<1$ & ns & - \\
\hline Matrix span simple & $<1$ & ns & - \\
\hline Matrix span complex & $<1$ & ns & - \\
\hline \multicolumn{4}{|l|}{ Dyscalculia * Dyslexia } \\
\hline Location span & 2.68 & ns & - \\
\hline Corsi-block simple & $<1$ & ns & - \\
\hline Corsi-block complex & $<1$ & ns & - \\
\hline Matrix span simple & $<1$ & ns & - \\
\hline Matrix span complex & $<1$ & ns & - \\
\hline
\end{tabular}

\section{Central executive}

Finally, the scores on the four tasks assessing central executive were entered into a multivariate analysis of variance (MANCOVA). The multivariate main effect for dyscalculia, $F(4,88)<1$, was not significant. In contrast, the multivariate main effect for dyslexia, $F(4$, $88)=5.79, \eta^{\uparrow}=.208, p<.000$, proved to be significant. The interaction of the two factors was 
not significant, $F(4,88)<1$. All univariate tests were presented in Table 4.2.5. For the dyslexia factor, univariate tests showed significant differences between groups on all central executive memory tasks.

Table 4.2.5 Central executive - Univariate Tests for the Factors Dyscalculia, Dyslexia, and the Dyscalculia * Dyslexia Interaction

\begin{tabular}{|c|c|c|c|}
\hline & $F$ & $p$ & $r^{2}$ \\
\hline \multicolumn{4}{|l|}{ Dyscalculia } \\
\hline Backward digit span & $<1$ & $\mathrm{~ns}$ & - \\
\hline Backward words span & 2.52 & $\mathrm{~ns}$ & - \\
\hline Double span & $<1$ & $\mathrm{~ns}$ & - \\
\hline Counting span & 1.35 & $\mathrm{~ns}$ & - \\
\hline \multicolumn{4}{|l|}{ Dyslexia } \\
\hline Backward digit span & 13.95 & .000 & .133 \\
\hline Backward words span & 5.17 & .025 & .054 \\
\hline Double span & 5.53 & .021 & .057 \\
\hline Counting span & 13.07 & .000 & .126 \\
\hline \multicolumn{4}{|l|}{ Dyscalculia * Dyslexia } \\
\hline Backward digit span & $<1$ & $\mathrm{~ns}$ & - \\
\hline Backward words span & $<1$ & $\mathrm{~ns}$ & - \\
\hline Double span & $<1$ & $\mathrm{~ns}$ & - \\
\hline Counting span & $<1$ & $\mathrm{~ns}$ & - \\
\hline
\end{tabular}

However, when we controlled for the influence of the phonological loop on the performance of the central executive, the differences in measures of central executive functioning between children with (RD and $A D+R D)$ and without $(A D$ and $C)$ dyslexia were no longer significant. Specifically, when ANCOVAs were used to control for performance on one-syllable word span forward in the word backward span task $\left(F(1,91)=1.53, \eta^{\uparrow}=.02, p>.05\right)$, for performance on images span in the double span task $\left(F(1,91)=1.22, \eta^{2}=.01, p>.05\right)$, for performance on digit span in the counting span task $\left(F(1,91)=1.21, \eta^{\uparrow}=.01, p>.05\right)$, and for performance on both digit span forward and nonword repetition in the word backward span 
task $\left(F(1,91)=3.65, \eta^{\uparrow}=.04, p>.05\right)$, no significant effects were found. Therefore we conclude that the predominant working memoy deficit in dyslexia is not in central executive but in phonological loop functioning.

In summary, children in the $\mathrm{RD}$ group and $\mathrm{C}$ group (without dyscalculia) outperformed children in the $\mathrm{AD}$ and $\mathrm{AD}+\mathrm{RD}$ groups (with dyscalculia) on all measures of visual-spatial memory. At the same time, children in the $\mathrm{AD}$ group and $\mathrm{C}$ group (without dyslexia) outperformed children in the $\mathrm{RD}$ and $\mathrm{AD}+\mathrm{RD}$ groups (with dyslexia) on all measures of phonological memory.

\section{Discussion}

The aim of the present study was to analyze the role of the different components of working memory in various learning disabilities defined according to ICD-10. To this end, a broad battery of working memory measures was used to assess phonological, visual-spatial, and central executive functioning in children with specific disorders of arithmetical skills, specific reading disorders, and mixed disorders of scholastic skills, and in a control group of normally achieving peers.

Altogether, results confirm the value of using a comprehensive battery of measures to assess the cognitive memory deficits of children with clinically relevant learning disorders. Whereas previous findings have been mixed, the presented direct comparison of different learning disabilities within a single study design provides broad support for distinct patterns of working memory deficits. For example, independent of differences in age and IQ, children with specific reading disorders were found to have marked impairments in the phonological loop, scoring significantly lower than their peers without reading disorders on all seven phonological working memory tasks. This result replicates findings from numerous empirical studies that have reported phonological deficits in children with dyslexia (e.g., Gathercole, Alloway, Willis, \& Adams, 2006; Helland \& Asbjørnsen, 2004; Kibby et al., 2004; Siegel \& Linder, 1984). Children with specific reading disorders also exhibited poor performance in tasks testing central executive functioning. Because all of the tasks selected to assess central executive functioning drew on the phonological loop, however, it was important to control its influence on performance in central executive tasks. Interestingly, the significant differences in all measures disappeared when phonological working memory scores were partialed out, indicating that the most relevant working memory deficit in children with specific reading disorders is the phonological impairment rather than deficits in central executive functioning. 
Because performances on all tasks involving the phonological subsystem are impaired, it seems reasonable to assume an isolated but massive impairment of this subsystem.

The consistently good results of children with reading and spelling disorders on visualspatial working memory tasks attest to the unimpaired functioning of the visual-spatial sketchpad in this population. This result is in line with the findings of Gould and Glencross (1990) and Kibby et al. (2004), who also found the visual-spatial slave system to function unimpaired in children with dyslexia. Based on these findings, it seems rather unlikely that visual processing and storage is impaired in children with developmental dyslexia.

A characteristic pattern of deficits was also found for children with specific disorders of arithmetic skills, namely a specific impairment in the functioning of the visual-spatial sketchpad. Children with arithmetic learning disorders scored markedly lower than their peers without such disorders on the visual-spatial working memory tasks. It was only in the simple version of the corsi-block task that the difference in performance did not reach statistical significance. Overall, all children performed higher on the simple task versions (corsi-block simple, matrix span simple) than on the complex task versions (corsi-block complex, matrix span complex). However, children with and without arithmetic learning disorders did not differ in terms of the magnitude of this difference: in both groups, it was approximately 2 sequence lengths for the simple and complex matrix span tasks and just under 1 sequence length for the corsi-block tasks. Thus, variation in the degree of complexity of these tasks does not help to explain the specific pattern of impairment in visual-spatial memory exhibited by children with arithmetic learning disorders. With regard to the theoretical distinction between a more static visual cache and a more dynamic inner scribe component of the visualspatial sketchpad (Pickering et al., 2001) it seems appropriate to pursue the question of whether the working memory deficiencies in children with dyscalculia are more prone to one or the other component. Because the performance of children with specific arithmetic disorders was, overall, much lower on the indicators measuring the dynamic component (location span, corsi-block complex) and the matrix span tasks measuring the static component, broad impairments in the visual-spatial sketchpad can be assumed. However, this conclusion contradicts the hypotheses of several researchers who assume the storage of dynamic spatial information -- rather than the entire visual-spatial sketchpad -- to be impaired (McLean \& Hitch, 1999; see also Passolunghi, 2006) in students with specific arithmetic learning disabilities. 
We did not find children with specific arithmetical disorders to show any impairment in phonological working memory. This finding is in line with the results of several other studies (Geary et al., 1999, 2000; McLean \& Hitch, 1999).

Surprisingly, in contrast to previous findings (Geary et al., 1991, 1999, 2000; Hitch \& McAuley, 1991; McLean \& Hitch, 1999; Passolunghi \& Siegel, 2001; Siegel \& Ryan, 1989; Swanson, 1993; Swanson \& Sachse-Lee, 2001; Wilson \& Swanson, 2001), we did not find evidence for impairments in the central executive functioning of children with specific arithmetic disorders. This discrepancy may be attributable to differences in the classification of arithmetic learning disorders. Whereas only children with average intellectual abilities were included in the present study, children with lower levels of intellectual ability were also classified as having specific arithmetic learning difficulties in studies such as those by Geary et al. (1999, 2000) and McLean and Hitch (1999). In addition, many previous studies with children with arithmetic disorders did not explicitely control for reading and spelling achievement. Therefore the possibility that some comorbid disabled (dyslexia and dyscalculia) children might be responsible for the reported poor central executive task performance cannot be ruled out. Another point that warrants mention is that the studies differ in their measures of central executive working memory. The measures of backward span, double span, and counting span administered in the present study assess the coordination of processing and storing information. Thus, the possibility that the weak arithmetic skills measured are attributable to deficits in other specific subfunctions like selective attention or retrieval from long-term memory cannot be excluded.

Children with impairments in just one domain clearly outperformed children with combined arithmetic and reading disorders on almost all tasks administered in the present study. The interaction between the factors dyscalculia and dyslexia was not significant, however. In other words, the present findings do not suggest independent patterns of deficits in these children (cf. Schuchardt et al., 2006; van der Sluis et al., 2005). Rather, the results indicate that these children exhibit both deficits, i.e., those found for specific disorders of arithmetic skills and for specific disorders of reading/writing, but to a greater extent.

To conclude, comparison of children with different learning disorders revealed marked differences in working memory functioning. It is therefore important for future research to apply strict criteria to distinguish between children with specific disorders of arithmetical skills, children with specific disorders of reading/writing, and children with mixed disorders 
of scholastic skills, because these three subgroups show differential deficits in cognitive functioning.

Although a number of implications might be warranted from our finding that specific learning disorders are associated with distinct deficits in working memory functioning, one of them seems to be of utmost practical importance from our point of view. If deficiencies in children's phonological loop are an important precursor of dyslexia, and if deficiencies in children's visual-spatial sketch-pad are a precursor of dyscalculia one might use this knowledge for an early identification of children at risk a long time before they meet the ICD10 criteria of learning disorders at about the age of 8 years or so. One advantage of such an early identification of children with a risk for specific learning disorders would be the opportunity to apply focused prevention programmes to them, which might reduce the severeness of the adumbrated learning disorder.

\section{Summary}

This study examines working memory functioning in children with specific developmental disorders of scholastic skills as defined by ICD-10. Ninety-seven $2^{\text {nd }}$ to $4^{\text {th }}$ graders with a minimum IQ of 80 were compared using a $2 \times 2$ factorial (dyscalculia vs. no dyscalculia; dyslexia vs. no dyslexia) design. An extensive test battery assessed the three subcomponents of working memory described by Baddeley (1986): phonological loop, visual-spatial sketchpad, and central executive. Children with dyscalculia showed deficits in visual-spatial memory; children with dyslexia showed deficits in phonological and central executive functioning. When controlling for the influence of the phonological loop on the performance of the central executive, however, the effect was no longer significant. Although children with both reading and arithmetic disorders were consistently outperformed by all other groups, there was no significant interaction between the factors dyscalculia and dyslexia.

Während in Studie 2 die Betrachtung isolierter Lernstörungen mit der kombinierten Schulleistungsstörung im Vordergrund stand, soll nun im Folgenden das Augenmerk lediglich auf Kinder mit umfassenden Lernstörungen gelegt werden. Gefragt wird hierbei nach der besonderen Rolle der Intelligenz im Zusammenhang mit schwachen Schulleistungen und Arbeitsgedächtnisdefiziten. Aus diesem Grund wird nun in der dritten Studie das Intelligenzniveau variiert und mit der Funktionstüchtigkeit des Arbeitsgedächtnisses in Beziehung gesetzt. 


\subsection{Studie 3: Zeigen Kinder mit umfassenden Schulleistungsschwierigkeiten in Abhängigkeit von der Intelligenz unterschiedliche Arbeitsgedächtnisdefizite? ${ }^{3}$}

Working memory deficits are widely being discussed and identified as possible causal factors underlying learning disabilities. Although various models of working memory have been developed the British model by Baddeley (1986) has proved a particularly useful theoretical tool in numerous studies on learning disabilities. According to this model working memory comprises three components: the modality-free central executive, which is a kind of supervisory system that serves to control and regulate the cognitive processes occurring, and two slave systems, the phonological loop and the visual-spatial sketchpad. The functions of the central executive that have been identified by Baddeley (1996) include coordinating the slave systems, focusing and switching attention, and retrieving representations from long-term memory. The two slave systems perform modality-specific operations. Verbal and auditory information are stored temporarily and processed in the phonological loop. Two components of the phonological loop are distinguished: the phonological store and the subvocal rehearsal process. The visual-spatial sketchpad is concerned with remembering and processing visual and spatial information; it comprises a visual cache for static visual information and an inner scribe for dynamic spatial information (Logie, 1995; Pickering, Gathercole, Hall, \& Lloyd, 2001).

Research has provided numerous indications that specific learning disabilities are associated with impairments in working memory (Alloway \& Gathercole, 2006; Pickering, 2006a). There is considerable evidence that children with specific reading disabilities have deficits in phonological processing and storage (Pickering, 2006b; Swanson, 2006; Vellutino, Fletcher, Snowling, \& Scanlon, 2004), and there is evidence to suggest that they also experience deficits in central executive functioning (Landerl, Bevan, \& Butterworth, 2004; Pickering \& Gathercole, 2005). There are relatively few reports of impairments in the visualspatial working memory of reading disabled children, however (Kibby, Marks, Morgan, \& Long, 2004; Pickering, 2006b).

Empirical findings of children with specific arithmetic learning disabilities are also available for all three domains of working memory (Passolunghi, 2006). The central executive seems to be particularly impaired (Geary Hamson, \& Hoard, 2000; Geary, Hoard, \& Hamson,

\footnotetext{
${ }^{3}$ Dieses Kapitel beruht auf einer Publikation, die bei der Zeitschrift Journal of Intellectual Disabilities Research erscheinen wird (Mähler, C. \& Schuchardt, K. (in press) Working memory functioning in children with learning disabilities: Does intelligence make a difference?).
} 
1999; Passolunghi \& Siegel, 2001; Swanson \& Sachse-Lee, 2001), but findings on the phonological loop are inconsistent (see Swanson \& Sachse-Lee, 2001 vs. Geary et al.,1999, 2000 or Landerl et al., 2004). Thus, deficits in the phonological loop may not be a defining characteristic of children with arithmetic learning disabilities. Recent studies (Passolunghi, 2006; van der Sluis, van der Leij, \& de Jong, 2005) have also reported visual-spatial deficits in children with specific arithmetic disability (but see also Bull et al., 1999, and Geary et al., 2000).

Yet there is not much research with children who show specific developmental disorders of scholastic skills in both areas (arithmetical and reading and/or spelling skills). Either not enough information is given about the characteristics of the groups of learning disabled children to precisely define the children as suffering from a double deficit, or the studies were limited to a small number of working memory tasks and therefore could not yield a comprehensive assessment of the working memory system. Nevertheless, two studies seem adequate to report relevant results. Van der Sluis et al. (2005) found a deficit only in the central executive subsystem that could be interpreted as an addition of the minor deficits of either reading disabled or arithmetically disabled children. In a study from our own lab (Schuchardt, Mähler \& Hasselhorn, in press) a broad battery of working memory measures was used to assess phonological, visual-spatial, and central executive functioning in children with specific disorders of arithmetical skills, specific reading disorders, and mixed disorders of scholastic skills, and in a control group of normally achieving peers. Altogether, results confirm the value of using a comprehensive battery of measures to assess the cognitive memory deficits of children with clinically relevant learning disorders. Whereas previous findings have been mixed, direct comparison of different learning disorders within a single study design provides broad support for distinct patterns of deficits. Children with impairments in just one domain clearly outperformed children with combined arithmetic and reading disorders on almost all tasks administered in the present study. The results indicate that these children exhibit both deficits, i.e., those found for specific disorders of arithmetic skills and for specific disorders of reading/writing, and to a greater extent (see also Pickering \& Gathercole, 2004).

With regard to intellectual disabilities (low intelligence) working memory performance seems to depend heavily on the severity of the intellectual disability (Henry, 2001). While children at borderline of mental retardation only showed deficits in phonological 
working memory, children with mild or moderate intellectual disabilities were characterized by overall deficits in the different subsystems of working memory. Therefore working memory functioning seems to be strongly related to mental age and is consistent with a developmental delay account of mild intellectual disabilities (Henry, 2002; Van der Molen, Van Luit, Jongmans \& Van der Molen, 2007). Recently we obtained similar results (overall deficit) in a study with children of subnormal intelligence (IQ 55-85), the severest deficit being located in phonological working memory (Hasselhorn \& Mähler, 2007; Mähler, 2007).

The diagnosis "mixed disorder of scholastic skills" (ICD-10 F81.3) is given for a category of disorders in which both arithmetical and reading or spelling skills are significantly impaired but in which the disorder is not explicable in terms of general mental retardation or inadequate schooling. The essential criterion is the discrepancy between (normal) intelligence and (sub-average) performance in standardized tests of school achievement. Children with sub-average school achievement and sub-average intellectual development are supposed to suffer from intellectual disabilities rather than from learning disabilities and therefore do not receive this diagnosis. The open question is whether these two groups are characterized by different cognitive functioning, especially by distinct patterns of working memory functioning.

Therefore two questions where addressed:

(1) Are there specific working memory deficits underlying learning disabilities?

(2) Does intelligence make a difference, i.e. are there differences in working memory between learning disabled children with normal vs. sub-normal levels of intelligence?

\section{Methods}

Participants. Three groups of children participated in the study. Twenty-seven children received the diagnosis "mixed disorder of scholastic skills" (MDSS-group, ICD-10 F81.3, discrepancy between normal intelligence and sub-average scholastic skills). Another twentyseven children with comparable learning disabilities did not meet the diagnostic criteria due to the lack of discrepancy between scholastic skills and intelligence (intellectual disability group, i.e. ID-group, IQ 55-85). These two groups of learning disabled children were compared to a control group (C-group) of twenty-seven children without any specific developmental disorders of scholastic skills. Children from grades two, three or four with German as native language were included. The two groups of children with learning 
disabilities were recruited from our counseling center; all of them attended regular primary schools but did not reach sufficient achievement.

All children were screened with standardized tests of intellectual ability, spelling, reading and arithmetic. We administered the full IQ scale from the German version of the Kaufman Assessment Battery for Children (K-ABC, Melchers \& Preuß, 2001) to assess general intelligence, the score for nonverbal holistic thinking was used for matching the groups. We did not use the total IQ score in order to eliminate the K-ABC-subtests assessing sequential information processing similar to working memory measures. Spelling abilities were assessed by the Weingartener spelling tests for $2^{\text {nd }}$ and $3^{\text {rd }}$ graders (WRT 2+, Birkel, 1994a; WRT 3+, Birkel, 1994b) and the Westermann spelling test for $4^{\text {th }}$ graders (WRT $4 / 5$, Rathenow, 1980). In both of these standardized German tests, children insert dictated words into given sentences. Text reading was tested using the Salzburg Reading and Spelling Test (SLT, Landerl, Wimmer \& Moser, 1997). Mathematical skills were assessed using standardized German mathematics tests for $2^{\text {nd }}, 3^{\text {rd }}$, and $4^{\text {th }}$ graders (DEMAT $2+$, Krajewski, Liehm, \& Schneider, 2004; DEMAT 3+, Roick, Gölitz, \& Hasselhorn, 2004; DEMAT 4, Gölitz, Roick, \& Hasselhorn, 2006). These multi-component tests include computation problems, word problems, and geometry problems.

Table 4.3.1 summarizes the most relevant data to describe the three subgroups.

Table 4.3.1 Sex distribution and Means (Standard Deviations) of Age, K-ABC IQ, WRT Spelling T-Scores, SLT Text Reading T-Scores, and DEMAT Mathematics T-Scores by Subgroup

\begin{tabular}{lccc}
\hline & $\begin{array}{c}\text { ID } \\
(n=27)\end{array}$ & $\begin{array}{c}\text { MDSS } \\
(n=27)\end{array}$ & $\begin{array}{c}\text { Controls } \\
(n=27)\end{array}$ \\
\hline Sex (m/f) & $11 / 16$ & $13 / 14$ & $13 / 14$ \\
Age (months) & $106.63(12.45)$ & $107.63(12.35)$ & $107.59(10.58)$ \\
K-ABC-IQ & $75.44(7.49)$ & $100.08(8.25)$ & $101.04(11.44)$ \\
Spelling & $36.74(8.22)$ & $32.22(5.54)$ & $48.33(6.91)$ \\
Text Reading & $37.64(9.60)$ & $33.80(8.11)$ & $49.15(8.68)$ \\
Mathematics & $34.00(10.12)$ & $32.33(6.20)$ & $50.11(8.15)$ \\
\hline
\end{tabular}

Note. ID = children with lower intelligence and learning disabilities; MDSS = children with normal intelligence and mixed disorders of scholastic skills; $\mathrm{C}=$ normally achieving control children matched for chronological age.

The control group performed on average in all these measures. ID- and MDSS-groups showed the typical pattern of deficits in spelling, reading and mathematics, ID children performing better but still on a sub-average level $(\mathrm{T}<40)$ in spelling and reading. The ID 
group is defined by the lower IQ-score. There is a significant difference in intelligence between the two learning disabled groups $(t(44)=10.17, p=.00)$ and between the ID- and control group $(t(46)=8.53, p=.00)$, whereas the MDSS-group is not different from the control group $(t(44)=.33, \mathrm{n}$. s.).

Tasks. Working memory was assessed by a battery of 14 tasks: 5 phonological tasks (memory span for digits, one-syllable and three-syllable words, one-syllable nonwords, nonword repetition), 5 visual-spatial tasks (memory span for locations, matrix span simple and complex, corsi-block simple and complex), 4 central executive tasks (double span, backward spans for one-syllable words and digits, counting span). Tasks were presented in a fixed order: location span, double span, one- and three-syllable word span, corsi-block simple and complex, nonword repetition, backward word span, backward digit span, counting span, matrix span simple and complex, digit span, one-syllable non-word span. A detailed description of all tasks follows below.

Phonological loop. The digit span is the conventional measure used to assess shortterm phonological capacity. A series of digits (1 to 9) was presented acoustically at a rate of one digit per second, starting with two and continuing up to a maximum of eight digits per sequence. Participants had to repeat the digits immediately in the given order. The onesyllable (e.g., Stern=star, Fisch=fish) and three-syllable word span tasks (e.g. Erdbeere=strawberry, Briefkasten=letterbox) and the one-syllable nonword span tasks (e.g., fen, sim) were presented in the same manner as in the digit span measure. The German nonword repetition task administered was developed by Hasselhorn and Körner (1997). Children had to repeat 24 word-like nonwords of 2, 3, or 4 syllables immediately after their presentation. Nonwords of different lengths were presented acoustically in random order. The number of correctly repeated nonwords was taken as the score for this task.

Visual-spatial sketchpad. In the location span task, children were shown a series of green dots at different locations on a $3 \times 3$ matrix and asked to recall these locations in the correct order. Corsi-block tasks were used to assess the dynamic spatial component of visualspatial working memory. The experimenter taps a sequence of red blocks on a grey board at the rate of one per second. The child then attempts to reproduce the sequence of taps in the correct order. We used two variations of the Corsi-block task: simple sequences involving 
short distances between blocks without path crossings, and complex sequences involving long distances between blocks with path crossings. A matrix span task was used to measure the static component of visual-spatial memory. Patterns of white and black squares in a $4 \times 4$ matrix were presented on the computer, beginning with two black squares and continuing up to a maximum of eight black squares. Immediately after presentation, children were asked to reproduce the pattern in an empty matrix. Two variations of this task were also implemented: a simple matrix span with the black squares arranged in simple patterns, and a complex matrix span with the black squares located at some distance from one another.

Central executive. The same items and procedures were used for the backward digit and word span tasks as for the forward spans, the only difference being that participants were required to recall the sequences of items in reverse order. Additionally, a double span task was implemented to assess the children's ability to coordinate the functioning of the phonological loop and the visual-spatial sketchpad (see also Towse \& Houston-Price, 2001 for justification of such a task). Pictures of well-known objects were presented in different locations on a $3 \times 3$ matrix. Children had to recall the pictures and their location in the order of presentation. The complex counting span task, a measure of storage and processing efficiency, was based on a task introduced by Case, Kurland, \& Goldberg (1982). Increasing series of maps (maximum eight maps) with yellow circles (target items) and squares (distractor items) were presented in a random, computer-generated pattern. Children were instructed to always count the number of circles. Finally, the experimenter asked the child to recall the number of circles counted on each map.

Stop criterion. We used the same stop criterion for all span tasks. The length of the sequences presented was increased gradually, beginning with a minimum of two, and increasing to a maximum of eight items. There were four trials at each sequence length. If a child succeeded on two successive trials of the same length, the task continued with the next span length. If a child failed on two successive trials of the same length, he or she was not presented with any further sequences of the same length, but with a sequence one item shorter. The dependent measure for all span tasks was the longest sequence of items repeated in correct order. Children were credited an extra 0.25 point if they repeated a further sequence of the same length correctly (e.g., a score of 5.25 was awarded if two of four 5-item sequences 
were recalled correctly, 5.5 if three of four sequences, and 5.75 if all four sequences were recalled correctly).

\section{Results}

Performance on the different memory span tasks was measured for the three groups of participants (ID, MDSS, C), and group comparisons were carried out separately for the three subsystems of working memory. The significance level of all analyses was set at alpha $=.05$. Table 4.3.2 informs about the performance of the children on the different tasks, sorted by subsystems of working memory (phonological loop, visual-spatial sketchpad and central executive).

Table 4.3.2 Means (Standard Deviations) for Working Memory Measures by Subgroup

\begin{tabular}{lccc}
\hline & ID & MDSS & C \\
\hline Phonological loop & $4.23(0.80)$ & $4.20(0.44)$ & $5.08(0.74)$ \\
Digit span & $3.95(0.67)$ & $3.86(0.54)$ & $4.51(0.66)$ \\
One-syllable word span & $3.34(0.41)$ & $3.35(0.48)$ & $3.73(0.49)$ \\
Three-syllable word span & $3.54(0.52)$ & $3.38(0.89)$ & $4.14(0.58)$ \\
One-syllable nonword span & $3.82(0.68)$ & $3.78(0.54)$ & $4.44(0.69)$ \\
Images span & $18.67(3.61)$ & $18.26(4.29)$ & $20.81(1.52)$ \\
Nonword repetition & & & \\
\hline Visual-spatial sketchpad & $4.20(0.70)$ & $4.61(0.79)$ & $5.18(0.84)$ \\
Location span & $4.57(1.59)$ & $5.30(1.20)$ & $5.85(1.21)$ \\
Corsi-block simple & $3.77(1.16)$ & $4.20(1.24)$ & $4.91(0.84)$ \\
Corsi-block complex & $5.12(1.73)$ & $5.86(1.34)$ & $6.78(1.33)$ \\
Matrix span simple & $3.11(0.91)$ & $3.50(1.39)$ & $4.79(1.56)$ \\
Matrix span complex & & & \\
\hline Central executive & $3.01(0.71)$ & $3.34(0.44)$ & $3.81(0.64)$ \\
Backward digit span & $3.15(0.46)$ & $3.26(0.37)$ & $3.65(0.54)$ \\
Backward word span & $3.07(0.80)$ & $3.53(0.66)$ & $4.04(0.69)$ \\
Double span & $3.31(0.73)$ & $3.23(0.61)$ & $4.29(0.87)$ \\
Counting span &
\end{tabular}

Note. ID = children with lower intelligence and learning disabilities; MDSS = children with normal intelligence and mixed disorders of scholastic skills; $\mathrm{C}=$ normally achieving control children matched for chronological age.

The first question of the study was what kind of deficits children with learning disabilities show compared to unimpaired controls. To answer this question we compared 
MDSS-children with controls (both groups with normal IQ) for each subsystem separately. The scores of the six tasks assessing phonological loop functioning were entered into a MANOVA. The multivariate main effect, $F(6,47)=5,83, p<.001$, proved to be significant. The univariate tests showed significant differences between groups on all phonological tasks (digit span: $F(1,52)=27.85, M S E=0.38, p<.001$; one-syllable word span: $F(1,52)=15.75$, $M S E=0.36, p<.001$; three-syllable word span: $F(1,52)=8.24, M S E=0.24, p<.01$; onesyllable nonword span: $F(1,52)=13.85, M S E=0.56, p<.00$; nonword repetition: $F(1,52)=$ $8.50, M S E=10.37, p<.01)$.

In the same way the scores of the five tasks assessing visual-spatial sketchpad were entered into a second MANOVA. Again the multivariate group effect, $F(5,48)=2.48, p<$ .05 , proved to be significant. The univariate tests of visual-spatial sketchpad revealed significant differences between groups on all visual-spatial memory tasks (location span: $F(1$, $52)=6.44, M S E=0.67, p<.05$; corsi-block complex: $F(1,52)=5.95, M S E=1.12, p<.05$; matrix span simple: $F(1,52)=6.38, M S E=1.78, p<.05$; matrix span complex: $F(1,52)=$ $10.42, M S E=2.18, p<.01)$ with the exception of the corsi-block simple task, $F(1,52)=2.76$, $M S E=1.46, p>.05$.

Third the scores of the four tasks assessing central executive were entered into a multivariate analysis of variance (MANOVA). The multivariate group effect, $F(4,49)=7.52$, $p<.001$, proved to be significant. Univariate tests showed significant differences between groups on all central executive memory tasks (digit backward span: $F(1,52)=9.96, M S E=$ $0.30, p<.01$; word backward span: $F(1,52)=9.68, M S E=0.21, p<.01$; double span: $F(1$, $52)=7.37, M S E=0.46, p<.01$; counting span: $F(1,52)=26.44, M S E=0.57, p<.001)$. In general results reveal an overall deficit in working memory of the children with learning disabilities (MDSS group).

The second and more interesting question of the study was whether normal versus subnormal levels of intelligence are a crucial factor for working memory performance among children with learning difficulties. As can easily be figured out from table 4.3.2, unexpectedly, there were no obvious differences between the two disabled groups. The statistical analysis (comparison between ID- and MDSS-group) was again performed for each working memory subsystem separately by MANOVA, but all multivariate group effects were not significant (phonological loop: $F(6,47)<1$; visual-spatial sketchpad: $F(5,49)<1$; central executive: $F(4,49)=2.44, p>.05$.). Therefore, the different levels of intelligence between 
the two groups of children with learning disabilities (ID vs. MDSS) did not correspond with differences in working memory performance.

\section{Discussion}

Children with general learning disabilities (impairment of arithmetic and reading/spelling scholastic skills) show deficits in all measured aspects of working memory functions. This result is in line with other studies (Pickering \& Gathercole, 2004) and may lead to the conclusion that these children are more severely impaired concerning working memory than children with either dyslexia or dyscalculia, a fact that might explain the broader learning disorder.

However, unexpectedly, there were no significant differences between the two disabled groups. Working memory functioning did not differ although there was an IQdifference of 23 points which is equivalent to more than 1.5 standard deviations. This result corroborates the notion that working memory is associated with learning disabilities irrespective of intelligence level. Support for this finding comes from other studies concluding that working memory skills, especially performance on complex memory tasks similar to our central executive tasks, put an important constraint on the acquisition of skill and knowledge in reading and mathematics. The impact of this constraint seems to be independent from intelligence (Gathercole, Alloway, Willis, \& Adams, 2006).

Following this argument we agree with Dyck et al. (2004), who doubt the validity of the discrepancy criterion for defining developmental disorders. According to their study, the magnitude of underachievement, measured in a standardized test that defines the relation to normal development is the most important criterion, if possible combined with knowledge about concurrent deficits in functionally related abilities. Consequently, learning disorders can be understood as a substantial scholastic underachievement associated with working memory deficits that explain the learning disorder. Actually there is an ongoing discussion about the appropriateness and justification of the criterion of discrepancy for the diagnosis of learning disorders, especially "mixed disorders of scholastic skills" (Fletcher, Morris \& Lyon, 2003; Francis et al., 2005; Kavale \& Forness, 2003). Performance profiles of children with specific learning disabilities and children with more general intellectual disabilities seem to differ in some ways and are very similar in other aspects (as for example working memory in this study) and therefore do not provide a reason to rely on the criterion of discrepancy. Furthermore, intervention studies did not yield specific outcomes that could be attributed to 
differences in intelligence (Weber, Marx \& Schneider, 2002). Taken together there is not much support for the clinical practice to treat learning disabled children with or without normal intelligence as fundamentally different (i.e. to send them to different schools, to offer more support for learning disabled children with higher intelligence).

Nevertheless, our results cannot support the notion that working memory and intelligence are independent form one another. There is ample evidence that there is a strong relation between the two constructs, even that working memory is a strong predictor of general fluid intelligence (Oberauer, Schulze, Wilhelm \& Süß, 2005). Different levels of intelligence come along with different working memory profiles (Henry, 2001). However, the aim of our study was not, to predict intelligence or to predict working memory but to explain learning disabilities. Therefore we do not want to suggest that similar deficits in working memory in our two disabled groups will justify the prediction of similar development and similar coping with the learning disorders. Instead, we must leave the question about the predictive validity of working memory deficits for scholastic achievement open to future research.

\section{Summary}

Children with learning disabilities are identified by their severe learning problems and their deficient school achievement. As long as these children perform on average in an intelligence test and a significant discrepancy between (normal) intelligence and (sub-average) reading-, writing- and mathematical achievement in standardized tests is observed, the focus is on the disorders of scholastic skills. However, children with sub-average school achievement and sub-average intellectual development are supposed to suffer from intellectual disabilities rather than from learning disabilities. The open question is whether these two groups are characterized by different cognitive functioning. The present study explored several functions of working memory. A working memory battery with tasks for the phonological loop, the visual-spatial sketchpad and central executive skills was presented in individual sessions to 27 children with learning disabilities and normal IQ, 27 children with learning disabilities and low IQ, and a control group of 27 children with regular school achievement and normal IQ.

Results reveal an overall deficit in working memory of the two groups with learning disabilities compared to the control group. However, unexpectedly, there were no differences between the two disabled groups. This finding does not support the notion of discrepant cognitive functioning due to differences in intelligence of these two groups. In the ongoing 
discussion about the role of intelligence, especially about the postulated discrepancy between intelligence and school achievement for diagnosis and special education, our findings might lead to rethink the current practice of treating these two groups as fundamentally different. 


\section{Resümee und Ausblick}

Die Suche nach Ursachen und Interventionsmöglichkeiten bei Kindern mit Lernstörungen hat sich in den letzten Jahren $\mathrm{zu}$ einem sehr regen Forschungsgebiet entwickelt, an dem zahlreiche Fachrichtungen wie die Psychologie, Pädagogik, Neurobiologie oder auch Medizin beteiligt sind. Eine weit reichende Forschungstradition beschäftigt sich vornehmlich mit der Identifikation funktioneller Besonderheiten, die möglicherweise mit Beeinträchtigungen des Lernens in ursächlichem Zusammenhang stehen. Von großer Bedeutung ist hierbei die Frage nach basalen kognitiven Funktionsdefiziten, die spezifische Lernstörungen erklären und voneinander abgrenzen können. Innerhalb dieses Forschungsfeldes nimmt die Informationsverarbeitungsfähigkeit eine besondere Rolle ein, wobei vor allem Gedächtnisleistungen im Zentrum des Interesses stehen. Hierbei wird dem Arbeitsgedächtnis als Mechanismus der Verarbeitung und kurzfristigen Speicherung von Informationen eine besondere Bedeutung zugemessen. Viele Studien weisen auf die Bedeutsamkeit des Arbeitsgedächtnisses als wesentliche kognitive Grundfunktion der Rechen- und Schriftsprachleistungen hin (Alloway \& Gathercole, 2006; Pickering, 2006a). Häufig wird dabei das britische Arbeitsgedächtnismodell von Baddeley (1986) zugrunde gelegt, da es sich bereits in vielen Untersuchungen als theoretisches Rahmenmodell zur Analyse spezifischer Beeinträchtigungen bei Lernstörungen als nützlich erwiesen hat. So wurde das Modell über die Jahre mehr und mehr ausdifferenziert und hinsichtlich der verschiedenen Funktionen spezifiziert. Darüber hinaus wurden vermehrt Anstrengungen unternommen, Arbeitsgedächtnisleistungen detailliert, präzise und reliabel zu erfassen (Gathercole \& Pickering, 2000; Hasselhorn et al., 2003). Auch in dieser Forschungsarbeit wurde der Schwerpunkt auf kognitive Funktionsbeeinträchtigungen des Arbeitsgedächtnisses gelegt. Obwohl in den letzten Jahren zahlreiche Befunde zu Arbeitsgedächtnisauffälligkeiten bei Kindern mit schulischen Minderleistungen vorgelegt wurden, bestehen bei Betrachtung des aktuellen Forschungsstandes noch viele offene Fragen. Zu deren Beantwortung leistet diese Arbeit einen Beitrag.

Insgesamt wurde eine breite Auswahl von Lernstörungen in diese Forschungsarbeit aufgenommen, wobei sowohl die isolierte Lese-Rechtschreib- und die isolierte Rechenstörung als auch die Kombinierte Störung schulischer Fertigkeiten sowie die Lernbehinderung im Zentrum der Betrachtung standen. Der Untersuchung wurden dabei drei zentrale Fragen vorangestellt. Im Einzelnen wurde überprüft, ob (1) sich die Struktur des Arbeitsgedächtnisses bei Schulkindern mit und ohne Lernstörung unterscheidet, (2) welche Funktionsbereiche des 
Arbeitsgedächtnisses bei Kindern mit Lese-Rechtschreib- und/oder Rechenstörung beeinträchtigt sind und (3) ob Kinder mit umfassenden Schulleistungsschwierigkeiten in Abhängigkeit von der Intelligenz unterschiedliche Arbeitsgedächtnisdefizite zeigen. Zur Beantwortung dieser Forschungsfragen wurden drei aufeinander aufbauende Studien realisiert, deren Ergebnisse im Folgenden vorgestellt und die daraus erworbenen Erkenntnisse in das übergeordnete Themenfeld der funktionellen Besonderheiten der Informationsverarbeitung bei Lern- und Leistungsstörungen eingeordnet werden. Es folgen eine abschließende Bewertung des hier verfolgten Forschungsansatzes und ein Ausblick auf hieran anschließende weiterführende Forschungsaktivitäten.

Die erste Studie beschäftigte sich mit der grundlegenden Struktur des Arbeitsgedächtnisses bei Schulkindern. Im theoretischen Teil der vorliegenden Arbeit wurde das zugrunde liegende Arbeitsgedächtnismodell von Baddeley (1986) ausführlich vorgestellt und empirische Arbeiten zum Zusammenhang von Lernstörungen und Arbeitsgedächtnisleistungen überblicksartig aufgeführt. Es wurde deutlich, dass sich für Kinder mit Lese-Rechtschreibstörung, mit Rechenstörung, mit Kombinierter Störung schulischer Fertigkeiten und Lernbehinderung Leistungseinbußen in den verschiedenen Komponenten des Arbeitsgedächtnis zeigen lassen. Um die gefundenen Leistungsunterschiede als Hinweise auf funktionelle Beeinträchtigungen der jeweiligen Arbeitsgedächtniskomponenten interpretieren $\mathrm{zu}$ können, muss die Voraussetzung der strukturellen Gleichheit des Arbeitsgedächtnisses erfüllt sein. Obwohl in bisherigen Studien zur Suche nach Beeinträchtigungen in der Funktionstüchtigkeit des Arbeitsgedächtnisses bislang implizit von einer Äquivalenz der Arbeitsgedächtnisstruktur bei Kindern mit vs. ohne Lernstörungen ausgegangen wurde, lagen bislang noch keine Belege hierfür vor. Studie 1 beschäftigte sich daher erstmalig mit der empirischen Überprüfung der Strukturäquivalenz. Hierzu bearbeitete eine sehr umfangreiche Stichprobe von Kindern im Grundschulalter (129 Kinder mit und 116 Kinder ohne Lernstörung) verschiedene Aufgaben zur Erfassung der Leistungsfähigkeit des Arbeitsgedächtnisses.

Es konnte gezeigt werden, dass die drei Subkomponenten des Arbeitsgedächtnisses hoch miteinander korrelieren und dass im Rahmen konfirmatorischer Faktoranalysen hohe Faktorladungen der einzelnen Tests auf die entsprechenden Subkomponenten vorliegen. Der Vergleich des Zwei-Komponentenmodells mit dem Drei-Komponentenmodell des Arbeitsgedächtnisses machte deutlich, dass sich die Daten zwar auf das zweifaktorielle 
Modell anpassen lassen, aber - zumindest auf deskriptiver Ebene - nicht mit derselben Güte. So zeigt sich im Overall-Modelfit (RMSEA) ein nach Konventionen qualitativ schlechterer Fit. Dieser ist gemäß den Kriterien von Schermelleh-Engel, Moosbrugger und Müller (2003) mit .07 bzw. .08 nur ,adäquat“, während der für das Drei-Faktor-Modell als ,gut““ betrachtet werden kann. Gleichwohl weisen die partiellen $\chi^{2}$-Werte auf eine signifikant bessere Modellanpassung durch die Drei-Faktor-Struktur hin. Somit konnte bestätigt werden, dass, obwohl auch ein Zwei-Komponentenmodell eine akzeptable Anpassungsgüte aufweist, die des Drei-Komponentenmodells jedoch deutlich überlegen ist.

Zusammenfassend kann die eingangs aufgeworfene Frage nach strukturellen Unterschieden in der Arbeitsgedächtnisstruktur eindeutig beantwortet werden: Kinder mit und ohne Lernstörung verfügen über eine identische Struktur des Arbeitsgedächtnisses. Es zeigte sich, dass die der verwendeten Arbeitsgedächtnistestbatterie zugrunde liegende Vorstellung von drei unabhängigen Subkomponenten des Arbeitsgedächtnisses für beide Gruppen Gültigkeit besitzt.

Ziel der zweiten Studie war es, die Rolle der einzelnen Komponenten des Arbeitsgedächtnisses bei verschiedenen Lernstörungen zu analysieren und zu überprüfen, ob sich für Untergruppen spezifische Defizitmuster aufdecken lassen. Hierzu wurden drei Gruppen von Lernstörungen (Lese-Rechtschreibstörung, Rechenstörung und Kombinierte Störung schulischer Fertigkeiten) und eine Kontrollgruppe untersucht, die sich auf die vier Felder eines zweifaktoriellen Designs (Rechenstörung: ja vs. nein; Lese-Rechtschreibstörung: ja vs. nein) verteilen. Die insgesamt 97 Kinder der zweiten bis vierten Klassenstufe wurden mit einer sehr umfangreichen Testbatterie von 15 verschiedenen Arbeitsgedächtnisaufgaben untersucht.

Die Frage nach spezifischen Arbeitsgedächtnisdefiziten bei verschiedenen Lernstörungen kann mit einem beeindruckend klaren Ergebnismuster eindeutig beantwortet werden. So stellten sich bei den Kindern mit Lese-Rechtschreibstörung konsistent über alle Erhebungsmaße Beeinträchtigungen der phonologischen Schleife als Defizitschwerpunkt heraus. Es ließen sich ebenfalls schwache zentral-exekutive Leistungen feststellen, die jedoch eindeutig und vollständig auf phonologische Defizite zurückzuführen sind. Da sämtliche Leistungen, die das phonologische Subsystem beanspruchen, deutlich beeinträchtigt sind, liegt somit eine isolierte aber massive Beeinträchtigung dieses Subsystems vor, wobei sowohl der phonetische Speicher als auch der subvokale Rehearsalprozess betroffen zu sein scheinen. 
Ebenso zeichnete sich für Kinder mit Rechenstörung ein charakteristisches Defizitmuster in Form einer spezifischen Beeinträchtigung des visuell-räumlichen Notizblocks ab. Da die Leistungen der rechengestörten Kinder sowohl in den Indikatoren zur Messung der dynamischen Komponente als auch in den Indikatoren zur Messung der statischen Komponente deutlich schwächer ausgeprägt waren, scheint hier eine breite Beeinträchtigung des gesamten visuell-räumlichen Subsystems vorzuliegen.

Die Kinder mit kombinierten Leistungsstörungen zeigten in dieser Studie in fast allen Aufgaben deutlich schwächere Leistungen als Kinder mit Störungen in nur einem Schulleistungsbereich. Dennoch zeigte sich multivariat kein Interaktionseffekt hinsichtlich der beiden Faktoren Rechenstörung und Lese-Rechtschreibstörung, so dass die Ergebnisse dieser Studie nicht für ein unabhängiges Störungsmuster dieser Kinder sprechen, welches sie von Kindern mit Störungen in nur einem Leistungsbereich unterscheiden würde. Vielmehr weisen die Ergebnisse darauf hin, dass diese Kinder beide Defizite aufweisen, d.h. die für leserechtschreibgestörten Kinder charakteristischen Einbußen im phonologischen Subsystem sowie das spezifische visuell-räumliche Defizit der rechengestörten Kinder. Beide Arbeitsgedächtnisbereiche sind jedoch stärker beeinträchtigt als bei Kindern mit isolierten Leistungsstörungen.

Zusammenfassend lässt sich festhalten, dass sich das Modell des Arbeitsgedächtnisses (Baddeley, 1986) in dieser Studie als geeignet erwiesen hat, um kognitive Beeinträchtigungen bei Kindern mit Störungen im Lernen detailliert $\mathrm{zu}$ untersuchen. Wurden zu Arbeitgedächtnisbeeinträchtigungen der betroffenen Kinder bislang nur sehr heterogene Befunde berichtet, lassen sich durch strenge Selektionskriterien der untersuchten Stichproben und den direkten Vergleich der unterschiedlichen Lernstörungen innerhalb eines Designs sehr klar abgrenzbare Defizitmuster mit einer breiten Bestätigung aufdecken.

Die dritte Studie beschäftigte sich mit der Intelligenz als möglichen kognitiven Ursachenfaktor und verfolgte dabei die Frage, inwiefern sich Kinder mit generellen Schulleistungsschwierigkeiten in Abhängigkeit vom Intelligenzniveau in ihren Arbeitsgedächtnisfunktionen voneinander unterscheiden. Dieser Blick ist bislang neu und wurde erstmalig in dieser Studie aufgegriffen. Hierbei wurde überprüft, in welchen Arbeitsgedächtnisbereichen durchschnittlich (Kombinierte Störung schulischer Fertigkeiten) und unterdurchschnittlich (Lernbehinderung) intelligente Kinder mit umfassenden Lernschwierigkeiten sowohl im Schriftsprachbereich als auch im Rechnen Funktionsdefizite 
aufweisen und ob es substanzielle Unterschiede zwischen den beiden Subgruppen gibt. An der Studie nahmen insgesamt 27 Kinder mit kombinierten Schulleistungsstörungen, 27 Kinder mit diagnostizierter Lernbehinderung und 27 nach Alter und Geschlecht parallelisierte Kontrollkinder teil. Alle Kinder bearbeiteten eine umfangreiche Testbatterie von insgesamt 15 Aufgaben zu den drei Komponenten des Arbeitsgedächtnisses.

Auch in dieser Studie kann die eingangs aufgestellte Frage eindeutig beantwortet werden. Zusammenfassend wurden zwei Erkenntnisse gewonnen. Erstens wurde auch in dieser Studie deutlich, dass Kinder mit umfassenden Lernschwierigkeiten in den Bereichen der Rechtschreibung, des Lesens und des Rechnens eine massive Beeinträchtigung im Arbeitsgedächtnis aufzeigen, wobei sowohl die phonologische Schleife und der visuellräumliche Notizblock als auch die zentrale Exekutive betroffen sind. Somit zeigen die Kinder mit generellen Lernschwierigkeiten gravierendere Beeinträchtigungen im Arbeitsgedächtnis auf als Kinder mit isolierten Lernstörungen (Lese-Rechtschreibstörung und Rechenstörung). Zweitens zeigten sich trotz eines IQ-Unterschieds von über 20 Punkten zwischen den beiden lernschwachen Gruppen keine signifikanten Leistungsunterschiede im Arbeitsgedächtnis. Das bedeutet, dass umfassende schwache Schulleistungen unabhängig von der Intelligenz mit massiven Arbeitsgedächtnisdefiziten verbunden sind. Da nicht nur die modalitätsspezifischen Arbeitsgedächtniskomponenten sondern auch modalitätsübergreifende Arbeitsgedächtnisfunktionen betroffen sind, kann hier bei beiden Lernstörungen von einem generellen Arbeitsgedächtnisdefizit ausgegangen werden. Somit haben beide Gruppen im Hinblick auf die Funktionstüchtigkeit des Arbeitsgedächtnisses ein vergleichbares Informationsverarbeitungsdefizitmuster, wobei allgemein schwache intellektuelle Fähigkeiten als mögliche Ursache weitgehend ausgeschlossen werden können. Die Arbeitsgedächtnisfunktionen scheinen somit nicht geeignet $\mathrm{zu}$ sein, um diese beiden Lernstörungen voneinander $\mathrm{zu}$ unterscheiden. Es bleibt also offen, durch welche anderen kognitiven Merkmale Kinder mit Lernbehinderung und Kinder mit kombinierten Schulleistungsstörungen voneinander abgegrenzt werden können.

Als Gesamtfazit bleibt festzuhalten, dass die vorliegenden Analysen wichtige Erweiterungen bisheriger Studien in diesem Themenfeld darstellen. Dabei hat sich das Modell des Arbeitsgedächtnisses (Baddeley, 1986) auch in dieser Arbeit als hilfreich erwiesen, um kognitive Funktionsbeeinträchtigungen bei Kindern mit Störungen im Lernen detailliert zu analysieren. Hierzu wurde gezielt ein breites Spektrum spezifischer Arbeitsgedächtnisleistungen erfasst, die im ursächlichen Zusammenhang mit Lernstörungen 
zu stehen scheinen. Die für diese Arbeit zusammengestellte Arbeitsgedächtnistestbatterie ist insgesamt gut geeignet, die Struktur des dreigliedrigen Arbeitsgedächtnismodells umfassend abzubilden. Das gilt sowohl für lernbeeinträchtigte als auch unbeeinträchtigte Kinder. Auch haben sich die verschiedenen Arbeitsgedächtnisaufgaben als gut einsetzbar erwiesen, die subsystemspezifischen Arbeitsgedächtnisfunktionen für die Altersgruppe der Grundschulkinder zu untersuchen. Weiterhin lässt sich aus den Befunden der hier vorgelegten Studien schließen, dass sich bei sorgfältig, anhand strenger Kriterien ausgewählten Stichproben von Kindern mit verschiedenen Lernstörungen spezifische und sehr prägnante Ergebnismuster finden lassen. Bislang wurden recht heterogene und widersprüchliche Befunde zu Arbeitsgedächtnisbeeinträchtigungen und Lernschwierigkeiten im Schriftsprachbereich und im Rechnen berichtet. Gründe hierfür sind möglicherweise in der geringen Vergleichbarkeit der bisherigen Studien zu suchen. So wurden u.a. unterschiedliche Klassifikationskriterien für Lernstörungen angelegt. Oftmals stellten Leistungen im untersten Quartil das entscheidende Kriterium dar. Auch wurde der Einfluss der Intelligenz nur selten kontrolliert bzw. eine kritische Diskrepanz zu den schulischen Minderleistungen als Diagnosekriterium herangezogen. Demgegenüber sind die Ergebnisse dieser Arbeit zu klinisch deutlich ausgeprägten Störungsbildern (nach den Kriterien der ICD-10) sehr eindeutig: verschiedene Lernstörungen hängen mit spezifischen und voneinander abgrenzbaren Arbeitsgedächtnisproblemen zusammen. So lassen sich phonologische Arbeitsgedächtnisdefizite bei Störungen im Lesen und Schreiben, visuell-räumliche Arbeitsgedächtnisdefizite bei Störungen im Rechnen und phonologische, visuell-räumliche und zentral exekutive Arbeitsgedächtnisdefizite bei generellen Schulleistungsstörungen beobachten. Darüber hinaus spielt der Ausprägungsgrad des Intelligenzniveaus, anders als bisher vermutet, keine wesentliche Rolle im Zusammenhang mit spezifischen Arbeitsgedächtnisbeeinträchtigungen bei Lernstörungen. Den Ergebnissen dieser Arbeit zufolge muss angenommen werden, dass Begrenzungen in der Funktionstüchtigkeit der Arbeitsgedächtnisfunktionen einen wichtigen Verursachungsfaktor für die Ausbildung verschiedenster Lernstörungen darstellen. 


\section{Anregungen für weiterführende Forschungsarbeiten}

Die dargestellten Ergebnisse werfen in der Folge aber auch neue Fragen auf und regen zu weiterführenden Forschungsarbeiten an. So ist es bislang noch ungeklärt, ob der Ansatz der differenziellen Analyse der Arbeitsgedächtnisfunktionen sich auch als hilfreich erweist, um verschiedene Subgruppen von spezifischen Lernstörungen voneinander abzugrenzen. Beispielsweise ist für Kinder mit unterschiedlichen Beeinträchtigungen des Schriftspracherwerbs noch unbeantwortet, welche Arbeitsgedächtniseinschränkungen für Kinder mit isolierten Rechtschreibschwierigkeiten, mit isolierten Leseschwierigkeiten und kombinierten Lese- und Rechtschreibschwierigkeiten verantwortlich sein könnten. Gleiches ließe sich auf auch die Analyse spezifischer kognitiver Funktionsbeeinträchtigungen bei Subgruppen der Rechenstörung übertragen (vgl. Geary, 1993, 1994; Rourke, 1993).

Weiterhin stellt sich die Frage, inwieweit der in dieser Arbeit dargestellte Befund, dass schwache Schulleistungen stärker mit Arbeitsgedächtnisdefiziten zusammenhängen als mit der Intelligenz, sich replizieren und auf andere Lernstörungen übertragen lässt. Dieser Aspekt ist sehr interessant und sollte durch die Verwendung spezifischer Forschungsdesigns weiter fortgeführt werden. Vorstellbar wäre in diesem Zusammenhang ein Vier-Gruppen-Vergleich, bei dem jeweils zwei hinsichtlich der Intelligenz und zwei hinsichtlich der Schulleistungen parallelisierte Gruppen in einem zweifaktoriellen Design miteinander verglichen werden. Sollte sich hierbei ergeben, dass sich die Kinder in Abhängigkeit ihrer Schulleistungen jedoch nicht in Abhängigkeit ihrer Intelligenz in den Arbeitsgedächtnisleistungen unterscheiden, wäre dies ein weiterer Beleg für die oben postulierte These der Unabhängigkeit von Intelligenz und Arbeitsgedächtnis, die folglich gegen die Anwendung des Diskrepanzkriteriums zur diagnostischen Bestimmung von Lernstörungen sprechen würde.

Das Fortbestehen von Lernstörungen über die Schuljahre hinweg lässt vermuten, dass sich auch die zugrunde liegenden kognitiven Defizite der betroffenen Kinder im Laufe der Entwicklung manifestieren. Wie u.a. Gathercole, Pickering, Ambridge und Wearing (2004) anführen, sind $\mathrm{ab}$ dem Grundschulalter keine qualitativen Veränderungen im Arbeitsgedächtnis mehr zu erwarten, lediglich eine allmähliche quantitative Steigerung der Arbeitsgedächtnisleistungen ist von der frühen Schulzeit bis zur Adoleszenz beobachtbar. Es wäre zu prüfen, ob sich vergleichbare Befunde auch bei lernbeeinträchtigten Kindern finden ließen. Wünschenswert wären hierzu längsschnittlich angelegte Studiendesigns, die Aufschluss über die Entstehung von Lernstörungen und damit zusammenhängenden Entwicklungsverläufen von Arbeitsgedächtnisfunktionen geben würden. Interessant ist in 
diesem Zusammenhang auch die Frage, ob sich die schulische Leistungsentwicklung von Kindern mit Lernstörungen durch die Funktionstüchtigkeit des Arbeitsgedächtnisses vorhersagen lässt.

\section{Praktische Relevanz}

Um die Frage der praktischen Relevanz der vorliegenden Ergebnisse für die Arbeit mit lernbeeinträchtigten Kindern $\mathrm{zu}$ beantworten, müssen sich diesem Beitrag der Grundlagenforschung Arbeiten der Anwendungsforschung anschließen. Zur Identifikation von Kindern mit Schwierigkeiten im Erwerb der schulischen Fertigkeiten könnte die Bestimmung eines charakteristischen Musters der Arbeitsgedächtnisleistungen differentialdiagnostisch von Bedeutung sein. Durch die gewonnene Kenntnis der Spezifität der Arbeitsgedächtnisbeeinträchtigungen kann genau erklärt werden, welche Kinder LeseRechtschreibprobleme, welche Kinder Rechenprobleme und welche Kinder umfassende Schwierigkeiten in beiden Bereichen ausbilden. Eine gezielte Diagnostik im Sinne einer Vorhersage wäre bereits vor dem Schuleintritt denkbar, um möglichst frühzeitig Defizite in den Arbeitsgedächtnisfunktionen zu bestimmen. Vorstellbar wäre die Entwicklung eines Testverfahrens, welches zur Identifikation von Risikokindern herangezogen werden könnte, für die aufgrund ihrer Arbeitsgedächtnisdefizite eine erhöhte Wahrscheinlichkeit für das Auftreten von Lernproblemen anzunehmen ist.

Eine Weiterführung des in dieser Arbeit verfolgten Forschungsansatzes besteht zudem darin, die gewonnenen Erkenntnisse über kognitive Verursachungsfaktoren von Lernstörungen für wirksame Präventions- und Interventionsmaßnahmen nutzbar zu machen. Wünschenswert wäre hierbei die Reduktion des Auftretens (Prävention) und des Ausmaßes (Intervention) der Lernprobleme der betroffenen Kinder. So könnten bisherige Präventionsprogramme, die hauptsächlich bereichsspezifische Vorläuferfertigkeiten trainieren (Krajewski, Nieding \& Schneider, 2005; Küspert \& Schneider, 2006; Plume \& Schneider, 2004), um bereichsübergreifende kognitive Aspekte ergänzt werden. Aus dem zentralen Befund, dass unterschiedliche Lernstörungen mit verschiedenen Defiziten in der Informationsverarbeitung einhergehen, ergibt sich die Notwendigkeit störungsspezifischer Behandlungsmöglichkeiten. Zukünftige Forschungsbemühungen sollten hier ansetzend geeignete Trainingsprogramme zur Überwindung der Schwierigkeiten entwickeln. Dabei sind zwei Wege möglich, den Schwierigkeiten der betroffenen Kinder zu begegnen. Zum einen könnten Auswirkungen der Defizite durch Förderung anderer relevanter Kompetenzen 
kompensiert werden. Ein Ansatzpunkt wäre beispielsweise die Erarbeitung förderlicher Lernstrategien, um das Lernen grundsätzlich zu optimieren. Auch lassen sich direkte Konsequenzen für die individuelle Unterstützung im Unterricht ableiten. So sollten beispielsweise Kinder mit den verschiedenen Lernstörungen von unterschiedlichen, d.h. visuellen oder sprachbasierten, Memorierhilfen profitieren. Es sollte daher fortführend der Frage nachgegangen werden, welche Kompensationsstrategien einen weniger erschwerten Erwerb der Lese-Rechtschreib- oder der Rechenfertigkeiten trotz bestehender Arbeitsgedächtnisdefizite ermöglichen. Hieraus ließen sich aufbauend wirksame Förderstrategien und spezifische der jeweiligen Art der Lernstörung angepasste Interventionsmaßnahmen entwickeln.

Zum anderen stellt sich aber auch die Frage, ob sich die gefundenen Defizite direkt beeinflussen lassen. Die Tatsache, dass die spezifischen Arbeitsgedächtnisdefizite nicht auf Strukturdefizite sondern auf Funktionsdefizite zurückführbar sind, birgt dabei die Hoffnung auf eine mögliche Trainierbarkeit von Informationsverarbeitungsfähigkeiten. Es sollte in zukünftigen Studien untersucht werden, ob sich die Funktionen des Arbeitsgedächtnisses gezielt trainieren lassen. Erste experimentelle Trainingsstudien bei Kindern mit Aufmerksamkeitsstörungen liefern hierzu vielversprechende Ergebnisse (Klingberg, Forssberg \& Westerberg, 2002; Klingberg et al., 2005).

Zusammengefasst besteht der wissenschaftliche Wert der vorliegenden Arbeit in der klaren Beantwortung bislang offener Forschungsfragen anhand von Untersuchungsdesigns nach zuvor festgelegten strengen Forschungskriterien. Aus den aufgeführten Ergebnissen ergeben sich darüber hinaus vielversprechende Ansätze für Vorhersage und Diagnostik, Prävention und Intervention bei Lernstörungen. 


\section{Zusammenfassung}

Das Ziel dieser Arbeit bestand in der differenziellen Analyse der Funktionstüchtigkeit des Arbeitsgedächtnisses (Baddeley, 1986) bei Kindern mit verschiedenen Lernstörungen. Hierzu wurden Grundschüler mit Lese-Rechtschreibstörung, Rechenstörung, Kombinierter Störung schulischer Fertigkeiten und Lernbehinderung anhand einer sehr umfangreichen Arbeitsgedächtnisbatterie mit vielen verschiedenen Indikatoren zu den drei Subsystemen phonologische Schleife, visuell-räumlicher Notizblock und zentrale Exekutive untersucht.

Im Rahmen dieser Arbeit wurde erstmalig empirisch nachgewiesen, dass Kinder mit und ohne Lernstörung über eine identische dreigliedrige Struktur des Arbeitsgedächtnisses verfügen. Erst vor dem Hintergrund der Evidenz dieser Strukturäquivalenz des Arbeitsgedächtnisses lassen sich gefundene Arbeitsgedächtnisdefizite lernbeeinträchtigter Kinder auch als Funktionsdefizite betrachten. Weiterhin konnten für verschiedene Störungsbilder spezifische und voneinander abgrenzbare Defizitmuster im Arbeitsgedächtnis aufgedeckt werden. So zeigten sich phonologische Arbeitsgedächtnisdefizite bei Störungen im Lesen und Schreiben, visuell-räumliche Arbeitsgedächtnisdefizite bei Störungen im Rechnen und phonologische, visuell-räumliche und zentral exekutive Arbeitsgedächtnisdefizite bei umfassenden Schulleistungsstörungen (Kombinierte Störung schulischer Fertigkeiten und Lernbehinderung). Eine weiterführende Analyse zeigte auf, dass sich Kinder mit kombinierter Schulleistungsstörung und Lernbehinderung nicht in ihren Arbeitsgedächtnisleistungen unterscheiden. Somit sind generelle Lernstörungen unabhängig vom Intelligenzniveau mit massiven Arbeitsgedächtnisdefiziten verbunden.

Insgesamt deuten die vorliegenden Ergebnisse darauf hin, dass die Untersuchung grundlegender kognitiver Funktionen im Rahmen des Arbeitsgedächtnismodells einen fruchtbaren Ansatz zum Verständnis der Lernstörungen darstellt. Hierauf aufbauend ergeben sich neue Perspektiven hinsichtlich einer Differenzialdiagnostik sowie verschiedenen Behandlungsmöglichkeiten bei lernbeeinträchtigten Kindern. 


\section{Literaturverzeichnis}

Alloway, T. P. \& Gathercole, S. E. (2006). Working memory and neurodevelopmantal disorders. Hove, UK: Psychology Press.

Alloway, T. P., Gathercole, S. E. \& Pickering, S. J. (2006). Verbal and visuospatial short-term and working memory in children: Are they separable? Child Development, 77, 16981716.

American Psychiatric Association. (2000). Diagnostic and Statistical Manual of Mental Disorders (4th ed.). Washington, DC: Autor.

Andersson, U. \& Lyxell, B. (2007). Working memory deficit in children with mathematical difficulties: A general or specific deficit? Journal of Experimental Child Psychology, 96, 197-228.

Atkinson, R. C. \& Shiffrin, R. M. (1968). Human memory: A proposed system and its control processes. In K. W. Spence and J. T. Spence (Eds.), The psychology of learning and motivation: Advances in research and theory (Vol. 2, pp. 89-195). New York: Academic Press.

Baddeley, A. D. (1986). Working memory. Oxford: University Press.

Baddeley, A. D. (1996). Exploring the central executive. Quarterly Journal of Experimental Psychology, 49, 5-28.

Baddeley, A. D. (1998). Human memory: Theory and practice (rev. ed.). Boston: Allyn and Bacon.

Baddeley, A. D. (2000). The episodic buffer: a new component of working memory? Trends in Cognitive Sciences, 4, 417-423.

Baddeley, A. D. (2006). Working memory: An overview. In S. J. Pickering (Ed.), Working memory and education. San Diego, CA: Academic Press.

Baddeley, A. D. \& Hitch, G. J. (1974). Working memory. In G. H. Bower (Ed.), The psychology of learning and motivation: Advances in research and theory (Vol. 2, pp. 89-195). New York: Academic Press.

Baddeley, A. D., Thomson, N. \& Buchanan, M. (1975). Word length and the structure of short-term memory. Journal of Verbal Learning and Verbal Behavior, 14, 575-589.

Badian, N. A. (1983). Arithmetic and nonverbal learning. In H. R. Myklebust (Ed.), Progress in learning disabilities (Vol. 5, pp. 253-264). New York: Grune \& Stration.

Birkel, P. (1994a). Weingartener Grundwortschatz Rechtschreib-Test für zweite und dritte Klassen (WRT 2+). Göttingen: Hogrefe.

Birkel, P. (1994b). Weingartener Grundwortschatz Rechtschreib-Test für dritte und vierte Klassen (WRT 3+).Göttingen: Hogrefe.

Bull, R., Johnston, R. S. \& Roy, J. A. (1999). Exploring the roles of the visual-spatial sketch pad and central executive in children's arithmetical skills: Views from cognition and developmental neuropsychology. Developmental Neuropsychology, 15, 421-442.

Case, R. D., Kurland, M. \& Goldberg, J. (1982). Operational efficiency and the growth of short-term memory span. Journal of Experimental Child Psychology, 33, 386-404.

D'Amico, A. \& Guarnera, M. (2005). Exploring working memory in children with low arithmetical achievement. Learning and Individual Differences, 15, 189-202. 
Daneman, M. \& Carpenter, P. A. (1980). Individual differences in working memory and reading. Journal of Verbal Learning and Verbal Behavior, 19, 450-466.

Deutscher Bildungsrat (1973). Empfehlungen der Bildungskommission zur pädagogischen Förderung behinderter und von Behinderung bedrohter Kinder und Jugendlicher. Boll - Bad Godesberg: Bundesdruckerei.

Dockrell, J. \& McShane, J. (1993). Childrens's learning difficulties: A cognitive approach. Cambridge: Blackwell.

Dyck, M. J., Hay, D., Anderson, M., Smith, L. M., Piek, J. \& Hallmayer, J. (2004). Is the discrepancy criterion for defining developmental disorders valid? Journal of Child Psychology and Psychiatry, 45, 979-995.

Eden, G. F. \& Stein, J. F. (1995). Verbal and visual problems in reading disability. Journal of Learning Disabilities, 28, 272-290.

Esser, G. \& Schmidt, M. (1993). Die langfristige Entwicklung von Kindern mit LeseRechtschreibschwäche. Zeitschrift für Klinische Psychologie, 2, 100-116.

Finucci, J. \& Childs, B. (1981). Are there really more dyslexic boys than girls? In A. Ansora, N. Geschwind, A. Galaburda, M. Albert \& N. Gartrell (Eds.), Sex differences in dyslexia (pp. 1-9). Towson, MD: Orton Dyslexia Society.

Flannery, K., Liederman, J., Daly, L. \& Schultz, J. (2000). Male prevalence for reading disability is found in a large sample of Black and White children free from ascertainment bias. Journal of the International Neuropsychological Society, 6, 433442 .

Fletcher, J. M., Morris, R. D. \& Lyon, G. R. (2003). Classification and Definition of Learning Disabilities: An Integrative Perspective. In H. L. Swanson, K. R. Harris \& S. Graham (Eds.), Handbook of Learning Disabilities (pp. 30-56). New York: Guilford.

Francis, D. J., Fletcher, J. M., Stuebing, K. K., Lyon, G. R., Shaywitz, F. A. \& Shaywitz, S. E. (2005). Psychometric approaches to the identification of LD: IQ and achievement scores are not sufficient. Journal of Learning Disabilities, 38, 98-108.

Fuchs, L. S., Compton, D. L., Fuchs, D., Paulsen, K., Bryant, J. D. \& Hamlett, C. L. (2005). The prevention, identification, and cognitive determinants of math difficulty. Journal of Educational Psychology, 97, 493-513.

Gathercole, S. E., Alloway, T. P., Willis, C. \& Adams, A. M. (2006). Working memory in children with reading disabilities. Journal of Experimental Child Psychology, 93, $265-$ 281.

Gathercole, S. E. \& Pickering, S. J. (2000). Assessment of working memory in six- and seven-year-old children. Journal of Educational Psychology, 92, 377-390.

Gathercole, S. E. \& Pickering, S. J. (2001). Working memory deficits in children with special educational needs. British Journal of Special Education, 28, 89-97.

Gathercole, S. E., Pickering, S. J., Ambridge, B. \& Wearing, H. (2004). The structure of working memory from 4 to 15 years of age. Developmental Psychology, 40, 177-190.

Gathercole, S. E., Willis, C., Baddeley, A. D. \& Emslie, H. (1994). The children's Test of Nonword Repetition: A test of phonological working memory. Memory, 2, 103-127.

Gaupp, N. (2003). Dyskalkulie - Arbeitsgedächtnisdefizite und Defizite numerischer Basiskompetenzen rechenschwacher Kinder. Berlin: Logos. 
Geary, D. C. (2004). Mathematics and learning disabilities. Journal of Learning disabilities, 37, 4-15.

Geary, D. C., Brown, S. C. \& Samaranayake, V. A. (1991). Cognitive addition: A short longitudinal study of strategy choice and speed-of-processing differences in normal and mathematically disabled children. Developmental Psychology, 27, 787-797.

Geary, D. C., Hamson, C. O. \& Hoard, M. K. (2000). Numerical and arithmetical cognition: A longitudinal study of process and concept deficits in children with learning disability. Journal of Experimental Child Psychology, 77, 236-263.

Geary, D. C., Hoard, M. K. \& Hamson, C. O. (1999). Numerical and arithmetical cognition: Patterns of functions and deficits in children at risk for a mathematical disability.Journal of Experimental Child Psychology, 74, 213-239.

Gelman. R. \& Gallistel, C. R. (1978). The child's understanding of number. Cambridge: Harvard University Press.

Gölitz, D., Roick, T. \& Hasselhorn, M. (2006). Deutscher Mathematiktest für vierte Klassen (DEMAT 4). Göttingen: Beltz.

Gould, J. H. \& Glencross, D. J. (1990). Do children with a specific reading disability have a general serial-ordering deficit? Neuropsychologia, 28, 271-278.

Gross-Tsur, V., Manor, O. \& Shalev, R. S. (1996). Developmental dyscalculia: Prevalence and demographic features. Developmental Medicine and Child Neurology, 38, 25-33.

Grube, D. (2008). Rechenschwäche. In W. Schneider \& M. Hasselhorn (Hrsg.), Handbuch der Pädagogischen Psychologie (S.642-652). Göttingen: Hogrefe.

Grube, D. \& Barth, U. (2004). Rechenleistung bei Grundschülern: Zur Rolle von Arbeitsgedächtnis und basalem Faktenwissen. Zeitschrift für Pädagogische Psychologie, 18, 245-248.

Grube, D. \& Hasselhorn (2006). Längsschnittliche Analysen zur Lese-, Rechtschreib- und Mathematikleistung im Grundschulalter: Zur Rolle von Vorwissen, Intelligenz, phonologischem Arbeitsgedächtnis und phonologischer Bewusstheit. In I. Hosenfeld \& F.-W. Schrader (Hrsg.), Schulische Leistung: Grundlagen, Bedingungen, Perspektiven (S.87-105). Münster: Waxmann.

Grünke, M. (2004). Lernbehinderung. In G. W. Lauth, M. Grünke \& J. C. Brunstein (Hrsg.), Interventionen bei Lernstörungen: Förderung, Training und Therapie in der Praxis (S. 65-77). Göttingen: Hogrefe.

Haffner, J., Zerahn-Hartung, C., Pfüller, U., Parzer, P., Strehlow, U. \& Resch, F. (1998). Auswirkungen und Bedeutung spezifischer Rechtschreibprobleme bei jungen Erwachsenen - empirische Befunde in einer epidemiologischen Stichprobe. Zeitschrift für Kinder- und Jugendpsychiatrie, 26, 124-135.

Hasselhorn, M. \& Gold, A. (2006). Pädagogische Psychologie. Erfolgreiches Lernen und Lehren. Stuttgart: Kohlhammer.

Hasselhorn, M., Grube, D. \& Mähler, C. (2000). Theoretisches Rahmenmodell für ein Diagnostikum zur differentiellen Funktionsanalyse des phonologischen Arbeitsgedächtnisses. In M. Hasselhorn, W. Schneider \& H. Marx (Hrsg.), Diagnostik von Lese-Rechtschreibschwierigkeiten (Tests und Trends - Jahrbuch der pädagogischpsychologischen Diagnostik, N.F., Bd. 1, S. 167-181). Göttingen: Hogrefe. 
Hasselhorn, M., Grube, D., Mähler, C., Zoelch, C., Gaupp, N. \& Schumann-Hengsteler, R. (2003). Differentialdiagnostik basaler Funktionen des Arbeitsgedächtnisses. In G. Ricken, A. Fritz \& C. Hofmann (Hrsg.), Diagnose: Sonderpädagogischer Förderbedarf (S. 277-291). Lengerich: Pabst.

Hasselhorn, M. \& Körner, K. (1997). Nachsprechen von Kunstwörtern: Zum Zusammenhang zwischen Arbeitsgedächtnis und syntaktischen Sprachleistungen bei Sechs- und Achtjährigen. Zeitschrift für Entwicklungspsychologie und Pädagogische Psychologie, 29, 212-224.

Hasselhorn, M. \& Mähler, C. (2006). Diagnostik von Lernstörungen. In M. Eid \& F. Petermann (Hrsg.), Handbuch der Psychologie, Band Diagnostik (S. 618-625). Göttingen: Hogrefe.

Hasselhorn, M. \& Mähler, C. (2007). Phonological working memory of children in two German special schools. International Journal of Disability, Development and Education, 54, 225-244.

Hasselhorn, M. \& Schuchardt, K. (2006). Lernstörungen: Eine kritische Skizze zur Epidemiologie. Kindheit und Entwicklung, 15, 208-215.

Hasselhorn, M. \& Schumann-Hengsteler, R. (2001). Arbeitsgedächtnis. In D. H. Rost (Hrsg.), Handwörterbuch Pädagogische Psychologie (2. Aufl., S. 17-22). Weinheim: Psychologie Verlags Union.

Hasselhorn, M., Seidler-Brandler, U. \& Körner, K. (2000). Ist das "Nachsprechen von Kunstwörtern" für die Entwicklungsdiagnostik des phonologischen Arbeitsgedächtnisses geeignet? In M. Hasselhorn, W. Schneider \& H. Marx (Hrsg.), Diagnostik von Lese-Rechtschreibschwierigkeiten (Tests und Trends - Jahrbuch der pädagogisch-psychologischen Diagnostik, N.F., Bd. 1, S. 119-133). Göttingen: Hogrefe.

Hasselhorn, M. \& Werner, I. (2000). Zur Bedeutung des phonologischen Arbeitsgedächtnisses für die Sprachentwicklung. In H. Grimm (Hrsg.), Enzyklopädie der Psychologie, Themenbereich C3: Sprache (S. 363-378). Göttingen: Hogrefe.

Helland, T. \& Asbjørnsen, A. (2004). Digit span in dyslexia: Variations according to language comprehension and mathematics skills. Journal of Clinical and Experimental Neuropsychology, 26, 31-43.

Henry, L. A. (2001). How does the severity of a learning disability affect working memory performance? Memory, 9, 233-247.

Henry, L. A. \& MacLean, M. (2002). Working memory performance in children with and without intellectual disabilities. American Journal on Mental Retardation, 107, 421432.

Hitch, G. J. \& McAuley, E. (1991). Working memory in children with specific arithmetical learning difficulties. British Journal of Psychology, 82, 375-386.

Howes, N. L., Bigler, E. D., Burlingame, G. M. \& Lawson, J. S. (2003). Memory performance of children with dyslexia. Journal of Learning Disabilities, 36, 230-246.

Jarvis, H. L. \& Gathercole, S. E. (2003). Verbal and non-verbal working memory and achievements on National Curriculum tests at 11 and 14 years of age. Educational and Child Psychology, 20, 123-140.

Jöreskog, K. \& Sörbom, D. (2005). LISREL 8.72 [software]. Chicago: Scientific Software International. 
Jurden, F. H. (1995). Individual differences in working memory and complex cognition. Journal of Educational Psychology, 87, 93-102.

Kane, M. J., Hambrick, D. Z., Tuholski, S. W., Wilhelm, O., Payne, T. W. \& Engle, R. Q. (2004). The generality of working-memory capacity: A latent-variable approach to verbal and visuo-spatial memory span an reasoning. Journal of Experimental Psychology: General, 133, 189-217.

Kavale, K. A. \& Forness, S. R. (2003). Learning Disability as a Discipline. In H. L. Swanson, K. R. Harris \& S. Graham (Eds.), Handbook of Learning Disabilities (pp. 76-93). New York: Guilford.

Kibby, M., Marks, W., Morgan, S. \& Long, C. (2004). Specific impairment in developmental reading disabilities: A working memory approach. Journal of Learning Disabilities, 37, 349-363.

Klauer, K. J. \& Lauth, G. W. (1997). Lernbehinderungen und Leistungsschwierigkeiten bei Schülern. In F. E. Weinert (Hrsg.), Psychologie des Unterrichts und der Schule (Enzyklopädie der Psychologie, Themenbereich D, Serie I, Pädagogische Psychologie, S. 701-738). Göttingen: Hogrefe.

Klicpera, C. \& Gasteiger-Klicpera, B. (1998). Psychologie der Lese- und Schreibschwierigkeiten - Entwicklung, Ursachen, Förderung (2. Aufl.). Weinheim: Beltz.

Klingberg, T., Fernell, E., Olesen, P., Johnson, M., Gustafsson, P., Dahlström, K., Gillberg, C. G., Forssberg, H. \& Westerberg, H. (2005). Computerized training of working memory in children with ADHD - A controlled, randomized, double-blind trial. Journal of the American Academy of Child and Adolescent Psychiatry, 44, 177-186.

Klingberg, T., Forssberg, H. \& Westerberg, H. (2002). Training of working memory in children with ADHD. Journal of Clinical and Experimental Neuropsychology, 24, 781-791.

Krajewski, K., Liehm, S. \& Schneider, W. (2004). Deutscher Mathematiktest für zweite Klassen (DEMAT 2). Göttingen: Beltz.

Krajewski, K., Nieding, G. \& Schneider, W. (2005). Mengen, zählen, Zahlen: Programm zur vorschulischen Förderung der Mengenbewusstheit von Zahlen und Zahlbeziehungen (MZZ). Unveröffentlichtes Manual, Lehrstuhl für Pädagogische und Entwicklungspsychologie, Universität Würzburg.

Kretschmann, R. (2007). Lernschwierigkeiten, Lernstörungen und Lernbehinderung. In J. Walter \& F. B. Wember (Hrsg.), Sonderpädagogik des Lernens. (S.4-32). Göttingen: Hogrefe.

Küspert, P. \& Schneider, W. (2006). Hören, lauschen, lernen - Sprachspiele für Vorschulkiner (5. überarbeitete Aufl.). Göttingen: Vandenhoeck \& Ruprecht.

Landerl, K., Bevan, A. \& Butterworth, B. (2004). Developmental dyscalculia and basic numerical capacities: A study of 8-9-year-old students. Cognition, 93, 99-125.

Landerl, K., Wimmer, H. \& Moser, E. (1997). Salzburger Lese- und Rechtschreibtest (SLRT). Bern: Huber.

Lauth, G. W. \& Schlottke, P. F. (2005). Lernbehinderte Kinder und Jugendliche. In P. F. Schlottke, S. Schneider, R. Silbereisen \& G. W. Lauth (Hrsg.), Enzyklopädie der Psychologie (S.327-348). Göttingen: Hogrefe. 
Lewis, C., Hitch, G. J. \& Walker, P. (1994). The prevalence of specific arithmetic difficulties and specific reading difficulties in 9- to 10-year-old boys and girls. Journal of Child Psychology and Psychiatry, 35, 283-292.

Liederman, J., Kantrowitz, L. \& Flannery, K. (2005). Male vulnerability to reading disability is not likely to be a myth: A call for new data. Journal of Learning Disabilties, 38, 109-129.

Logie, R. H. (1995). Visual-spatial working memory. Hove: Erlbaum.

Logie, R. H. \& Marchetti, C. (1991). Visuo-spatial working memory: Visual, spatial or central executive? In R. H. Logie \& M. Denis (Eds.), Mental images in human cognition (pp. 105-115). Amsterdam: North Holland.

Logie, R. H. \& Pearson, D. G. (1997). The inner eye and the inner scribe of visuo-spatial working memory: Evidence from developmental fractionation. European Journal of Cognitive Psychology, 9, 241-257.

Mähler, C. (2007). Arbeitsgedächtnisfunktionen bei lernbehinderten Kindern und Jugendlichen. Zeitschrift für Entwicklungspsychologie und Pädagogische Psychologie, 39, 97-106.

Mähler, C. \& Hasselhorn, M. (2003). Automatische Aktivierung des Rehearsalprozesses im phonologischen Arbeitsgedächtnis bei lernbehinderten Kindern und Erwachsenen. Zeitschrift für Pädagogische Psychologie, 17, 255-260.

Mähler, C. \& Schuchardt, K. (in press). Working memory functioning in children with learning disabilities: Does intelligence make a difference? Journal of Intellectual Disabilities Research.

Mazzocco, M. M. \& Myers, G. F. (2003). Complexities in identifying and defining mathematics learning disability in the primary school-age years. Annals of Dyslexia, 53, 218-253.

McLean, J. F. \& Hitch, G. J. (1999). Working memory impairments in children with specific arithmetic learning difficulties. Journal of Experimental Child Psychology, 74, 240260 .

Melchers, P. \& Preuß, U. (2001). Assessment Battery for Children (K-ABC), German version (5th ed.). Göttingen: Hogrefe.

Miles, T. R., Haslum, M. N. \& Wheeler, T. J. (1998). Gender ratio in dyslexia. Annals of Dyslexia, 48, 27-55.

Näslund, J. C. \& Schneider, W. (1996). Kindergarten letter knowledge, phonological skills, and memory processes: Relative effects on early literacy. Journal of Experimental Child Psychology, 62, 30-59.

Norman, D. A. \& Shallice, T. (1986). Attention to action: Willed and automatic control of behavior. In R. J. Davidson, G. E. Schwarts \& D. Shapiro (Eds.), Consciousness and self-regulation. Advances in research and theory (Vol. 4, pp. 1-18). New York: Plenum Press.

Oberauer, K., Schulze, R., Wilhelm, O. \& Süß, H.-M. (2005). Working memory and intelligence - their correlation and their relation: Comment on Ackerman, Beier, and Boyle (2005). Psychological Bulletin, 131, 61-65.

O’Shaughnessy, T. \& Swanson, H. L. (1998). Do immediate memory deficits in students with learning disabilities in reading reflect a developmental lag or deficit? A selective metaanalysis of the literature. Learning Disability Quarterly, 21, 123-148. 
Palmer, S. (2000). Phonological recoding deficit in working memory of dyslexic teenagers. Journal of Research in Reading, 23, 28-40.

Passolunghi, M. C. (2006). Working memory and arithmetic learning disability. In T. P. Alloway \& S. E. Gathercole (Eds.), Working memory and neurodevelopmental disorders (pp. 113-138). Hove, UK: Psychology Press.

Passolunghi, M. C. \& Siegel, L. S. (2001). Short-term memory, working memory, and inhibitory control in children with specific arithmetic learning disabilities. Journal of Experimental Child Psychology, 80, 44-57.

Pickering, S. J. (Ed.) (2006a). Working memory and education. San Diego, CA: Academic Press.

Pickering, S. J. (2006b). Working memory in dyslexia. In T. P. Alloway \& S. E. Gathercole (Eds.), Working memory and neurodevelopmental disorders (pp. 7-40). Hove, UK: Psychology Press.

Pickering, S. J. \& Gathercole, S. E. (2001). Working Memory Test Battery for Children (WMTB-C). London: The Psychological Corporation.

Pickering, S. J. \& Gathercole, S. E. (2004). Distinctive working memory profiles in children with special educational needs. Educational Psychology, 24, 393-408.

Pickering, S. J. \& Gathercole, S. E. (2005). Working memory deficits in dyslexia: Are they located in the phonological loop, visuospatial sketchpad or central executive? Manuscript under revision.

Pickering, S. J., Gathercole, S. E., Hall, M. \& Lloyd, S. A. (2001). Development of memory for pattern and path: Further evidence for the fractionation of visuo-spatial memory. The Quarterly Journal of Experimental Psychology, 54, 397-420.

Plume, E. \& Schneider, W. (2004). Hören, lauschen, lernen 2: Sprachspiele mit Buchstaben und Lauten für Kindern im Vorschulalter. Göttingen: Vandenhoeck \& Ruprecht.

Ramaa, S. \& Gowramma, I. P. (2002). A systematic procedure for identifying and classifying children with dyscalculia among primary school children in India. Dyslexia, 8, 67-85.

Rathenow, P. (1980). Westermann Rechtschreibtest 4/5 (WRT 4/5). Braunschweig: Westermann.

Reuhkala, M. (2001). Mathematical skills in ninth-graders: Relationship with visuospatial abilities and working memory. Educational Psychology, 21, 387-398.

Roebers, C. M. \& Zoelch, C. (2005). Erfassung und Struktur des phonologischen und visuellräumlichen Arbeitsgedächtnisses bei 4-jährigen Kindern. Zeitschrift für Entwicklungspsychologie und Pädagogische Psychologie, 37, 113-121.

Roick, T., Gölitz, D. \& Hasselhorn, M. (2004). Deutscher Mathematiktest für dritte Klassen (DEMAT 3+). Göttingen: Beltz.

Rosenquist, C., Conners, F. A. \& Roskos-Ewoldson, B. (2003). Phonological and visuospatial working memory in individuals with intellectual disability. American Journal of Mental Retardation, 108, 403-413.

Rourke, B. P. (1993). Arithmetic disabilities, specific and otherwise: A neuropsychological perspective. Journal of Learning Disabilities, 26, 214-226. 
Rutter, M., Caspi, A., Fergusson, D., Horwood, L. J., Goodman, R., Maughan, B., Moffitt, T. E., Meltzer, H. \& Carroll, J. (2004). Gender differences in developmental reading disability: New findings from four epidemiological studies. Journal of the American Medical Association, 291, 2007-2012.

Schermelleh-Engel, K., Moosbrugger, H. \& Müller, H. (2003). Evaluating the fit of structural equation models: Tests of significance and descriptive goodness-fit measures. Methods of Psychological Research Online, 8, 23-74.

Schmid, C., Zoelch, C. \& Roebers, C. (2008). Das Arbeitsgedächtnis von 4- bis 5-jährigen Kindern - Theoretische und empirische Analyse seiner Funktionen. Zeitschrift für Entwicklungspsychologie und Pädagogische Psychologie,40, 2-12.

Schneider, W. \& Näslund, J. C. (1993). The impact of early metalinguistic competencies and memory capacity on reading and spelling in elementary school. European Journal of Psychology of Education, 8, 273-288.

Schuchardt, K., Kunze, J., Grube, D. \& Hasselhorn, M. (2006). Arbeitsgedächtnisdefizite bei Kindern mit schwachen Rechen- und Schriftsprachleistungen. Zeitschrift für Pädagogische Psychologie, 20, 261-268.

Schuchardt, K., Mähler, C. \& Hasselhorn, M. (in press). Working memory deficits in children with different learning disabilities. Journal of Learning Disabilities.

Schuchardt, K., Roick, T., Mähler, C. \& Hasselhorn, M. (2008). Unterscheidet sich die Struktur des Arbeitsgedächtnisses bei Schulkindern mit und ohne Lernstörung? Zeitschrift für Pädagogische Psychologie und Entwicklungspsychologie,40, 147-151.

Schulte-Körne, G., Deimel, W., Jungermann, M. \& Remschmidt, H. (2003). Nachuntersuchung einer Stichprobe von lese-rechtschreibgestörten Kindern im Erwachsenenalter. Zeitschrift für Kinder- und Jugendpsychiatrie und Psychotherapie, 31, 267-276.

Schulz, A. (1995). Lernschwierigkeiten im Mathematikunterricht der Grundschule. Berlin: Paetec.

Schumann-Hengsteler, R., Strobl, M. \& Zoelch, C. (2004). Temporal memory for locations: On the encoding of spatiotemporal information in children and adults. In G. L. Allen (Ed.), Human spatial memory: remembering where (pp. 101-124). New York: Erlbaum.

Sekretariat der Ständigen Konferenz der Kultusminister der Länder in der Bundesrepublik Deutschland (2004). Sonderpädagogische Förderung in Schulen 1993-2002. Statistische Veröffentlichungen der Kultusministerkonferenz, Dokumentation Nr. 170. Bonn: Autor.

Shah, P. \& Miyake, A. (1996). The separability of working memory resources for spatial thinking and language processing: An individual differences approach. Journal of Experimental Psychology: General, 125, 4-27.

Shalev, R. S. \& Gross-Tsur, V. (2001). Developmental dyscalculia. Pediatric Neurology, 24, 337-342.

Shalev, R. S., Manor, O., Auerbach, J. \& Gross-Tsur, V. (1998). Developmental dyscalculia: What counts? Prospective 3-year follow up. Journal of Pediatrics, 133, 358-362.

Shaywitz, S. E., Escobar, M. D., Shaywitz, B. A., Fletcher, J. M. \& Makuch, R. (1992). Evidence that dyslexia may represent the lower tail of a normal distribution of reading ability. New England Journal of Medicine, 326, 145-150. 
Siegel, L. S. (1989). Why we do not need intelligence test scores in the definition an analyses of Learning disabilities. Journal of Learning Disabilities, 22, 514-515.

Siegel, L. S. \& Linder, B. A. (1984). Short-term memory processes in children with reading and arithmetic disabilities. Developmental Psychology, 20, 200-207.

Siegel, L. S. \& Ryan, E. B. (1989). The development of working memory in normally achieving and subtypes of learning disabled children. Child Development, 60, 973980.

Stanovich, K. E. (2005). The future of a mistake: Will discrepancy measurement continue to make the learning disabilities field a pseudoscience? Learning Disability Quarterly, 28, 103-106.

Statistisches Bundesamt DESTATIS (2004). Bildung und Kultur, Allgemeinbildende Schulen. Fachserie 11/Reihe 1.

Strehlow, U., Kluge, R., Möller, H. \& Haffner, J. (1992). Der langfristige Verlauf der Legasthenie über die Schulzeit hinaus: Katamnesen aus einer kinderpsychiatrischen Ambulanz. Zeitschrift für Kinder- und Jugendpsychiatrie, 20, 254-265.

Swanson, H. L. (1993). Working memory in learning disability subgroups. Journal of Experimental Child Psychology, 56, 87-114.

Swanson, H. L. (1999). Reading comprehension and working memory in learning-disabled readers: Is the phonological loop more important than the executive system? Journal of Experimental Child Psychology, 72, 1-31.

Swanson, H. L. (2006). Working memory and reading disabilities: Both phonological and executive processing deficits are important. In T. P. Alloway \& S. E. Gathercole (Eds.), Working memory and neurodevelopmental disorders (pp. 59-88). Hove, UK: Psychology Press.

Swanson, H. L. \& Sachse-Lee, C. (2001). A subgroup analysis of working memory in children with reading disabilities: Domain-general or domain-specific deficiency? Journal of Learning Disabilities, 34, 249-263.

Towse, J. N., \& Houston-Price, C. M. T. (2001). Combining representations in working memory: A brief report. British Journal of Developmental Psychology, 19, 319-324.

Van der Molen, M. J., Van Luit, J. E. H., Jongmans, M. J. \& Van der Molen, M. W. (2007). Verbal working memory in children with mild intellectual disabilities. Journal of Intellectual Disabilities Research, 51, 162-169.

Van der Sluis, S., van der Leij, A. \& de Jong, P. F. (2005). Working memory in Dutch children with reading- and arithmetic-related LD. Journal of Learning Disabilities, 38, 207-221.

Vellutino, F. R., Fletcher, J. M., Snowling, M. J. \& Scanlon, D. M. (2004). Specific reading disability (dyslexia): What have we learned in the past four decades. Journal of Child Psychology and Psychiatry, 45, 2-40.

Wagner, R. K. \& Torgesen, J. K. (1987). The nature of phonological processing and ist causal role in the acquisition of reading skills. Psychological Bulletin, 101, 192-212.

Weber, J. \& Marx, P. (2008). Lese-Rechtschreibschwierigkeiten. In W. Schneider \& M. Hasselhorn (Hrsg.), Handbuch der Pädagogischen Psychologie (S.631-641). Göttingen: Hogrefe. 
Weber, J., Marx, P. \& Schneider, W. (2002). Profitieren Legastheniker und allgemein leserechtschreibschwache Kinder in unterschiedlichem Ausmaß von einem Rechtschreibtraining? Psychologie in Erziehung und Unterricht,, 49, 56-70.

Wilson, K. M. \& Swanson, H. L. (2001). Are mathematics disabilities due to a domaingeneral or a domain-specific working memory deficit? Journal of Learning Disabilities, 34, 237-248.

Wocken, H. (2000). Leistung, Intelligenz und Soziallage von Schülern mit Lernbehinderungen. Zeitschrift für Heilpädagogik, 51, 492-304.

Wolf, M. \& Bowers, P. G. (1999). The double-deficit hypothesis for the developmental dyslexia. Journal of Educational Psychology, 91, 415-438.

World Health Organization (WHO). (2005). ICD: classification of mental and behavioural disorders: Clinical descriptions and diagnostic guidelines $\left(10^{\text {th }}\right.$ revision $)$. WHO: Geneva.

Zigler, E. \& Balla, D. (1982). Mental Retardation. The Developmental-DifferenceControversy. Hillsdale NJ: Lawrence Erlbaum Associates. 


\section{Anhang}

Protokollbogen für die verwendete Arbeitsgedächtnisbatterie 


\section{Protokollbogen}

Arbeitsgedächtnisbatterie

Name

Alter

Klasse

Testzeitpunkt :

Testleitung 


\section{TEZEK $1 \& 2$}

\subsection{Gedächtnisspanne für Bilder}

\begin{tabular}{|c|c|c|c|}
\hline & Reiz & $\mathrm{k}$ & $\mathrm{ik}$ \\
\hline 1 & Blatt Drache & & \\
\hline 2 & Tür Mond & & \\
\hline 3 & Flugzeug Schirm & & \\
\hline 4 & Auge Tasche & & \\
\hline 5 & Glocke Brille Koffer & & \\
\hline 6 & Schuh Baum Tasche & & \\
\hline 7 & Fuß Blume Auto & & \\
\hline 8 & Bleistift Tür Herz & & \\
\hline 9 & Tasche Sonne Hand Blatt & & \\
\hline 10 & Tische Herz Schuh Drachen & & \\
\hline 11 & Baum Auge Flugzeug Fuß & & \\
\hline 12 & Koffer Bleistift Haus Sonne & & \\
\hline 13 & Mond Glocke Tisch Auto Hand & & \\
\hline 14 & Haus Schirm Brille Drachen Baum & & \\
\hline 15 & Blume Schuh Flugzeug Bleistift Tür & & \\
\hline 16 & Herz Glocke Mond Fuß Blatt & & \\
\hline 17 & Hand Schirm Brille Tisch Auto Drachen & & \\
\hline 18 & Sonne Koffer Haus Bleistift Blume Fuß & & \\
\hline 19 & Auge Baum Tasche Glocke Herz Mond & & \\
\hline 20 & Schirm Blatt Tisch Flugzeug Hand Auto & & \\
\hline 21 & Drachen Koffer Glocke Blume Haus Bleistift Sonne & & \\
\hline 22 & Brille Schuh Tür Blatt Fuß Herz Tasche & & \\
\hline 23 & Tische Flugzeug Blume Hand Mond Auge Baum & & \\
\hline 24 & Auto Bleistift Glocke Haus Koffer Sonne Schuh & & \\
\hline
\end{tabular}

Bemerkungen

\subsection{Gedächtnisspanne für Lokationen}

\section{Kontrolltafel}

\begin{tabular}{|l|l|l|}
\hline 1 & 2 & 3 \\
\hline 4 & 5 & 6 \\
\hline 7 & 8 & 9 \\
\hline
\end{tabular}

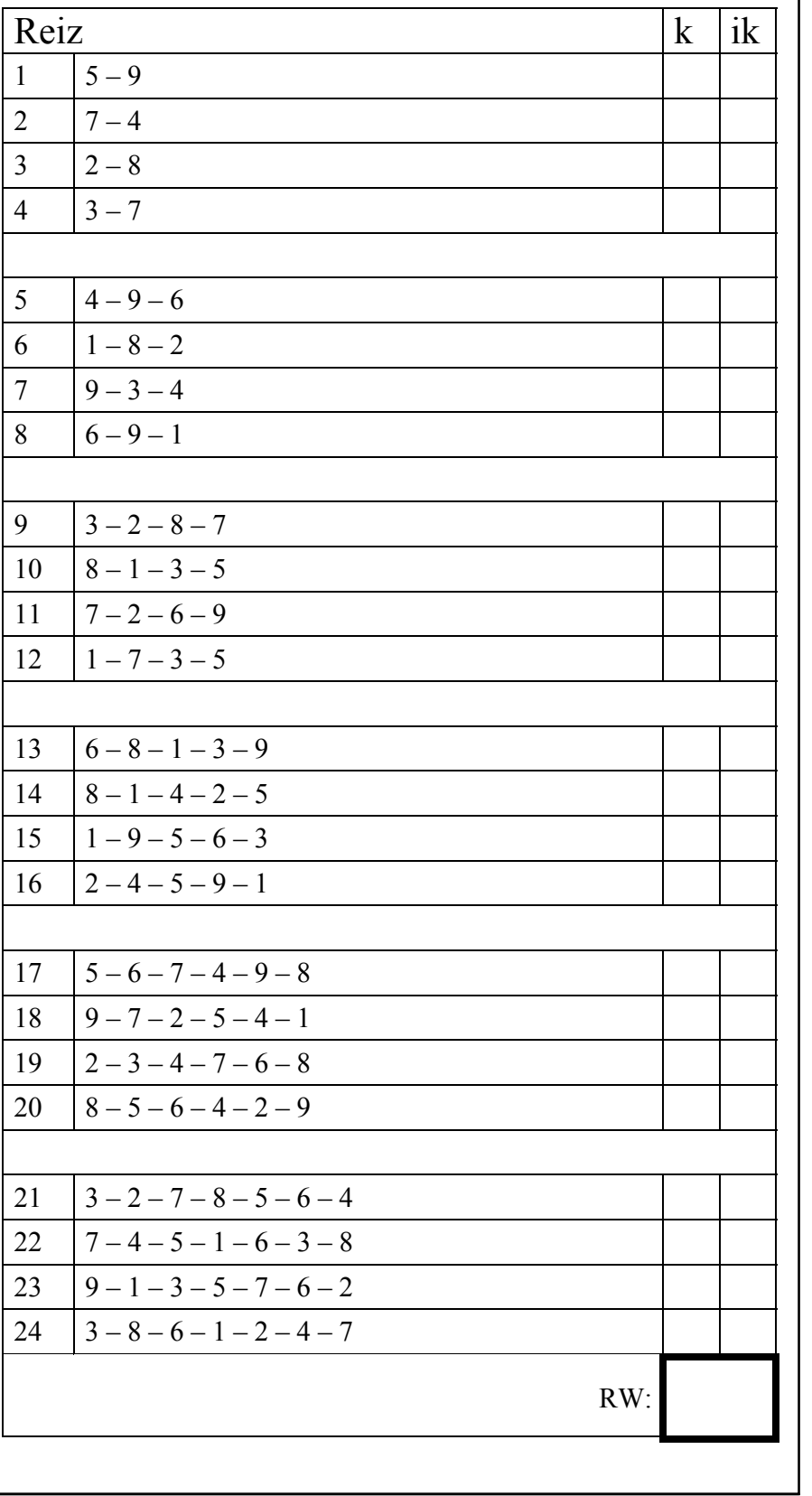




\section{TEZEK 3}

\subsection{Double Span}

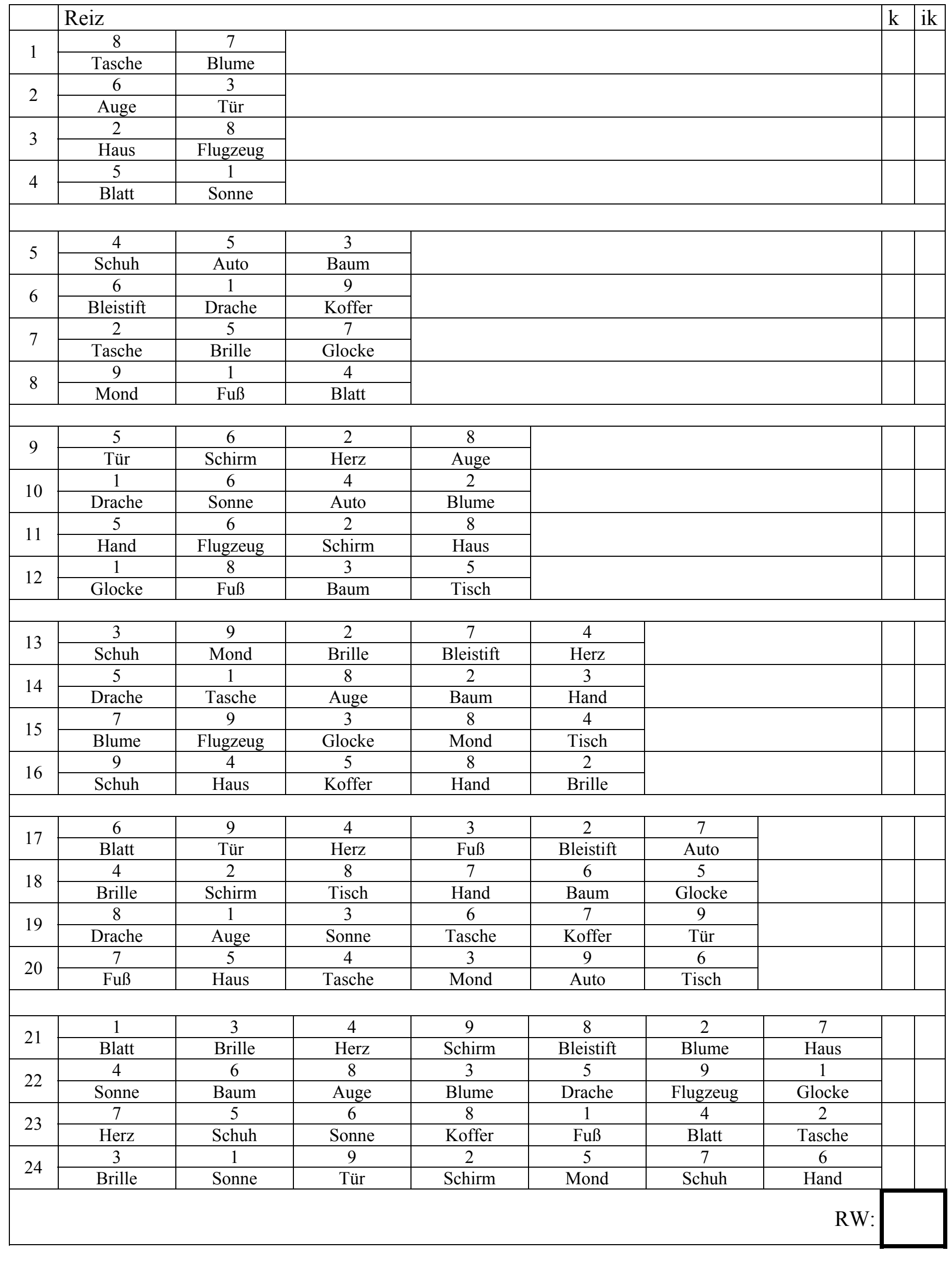




\section{Gedächtnisspanne vorwärts}

\subsection{Einsilbige Wörter}

\begin{tabular}{|l|l|l|l|}
\hline & Reiz & $\mathrm{k}$ & $\mathrm{ik}$ \\
\hline 2a & Topf Eis & & \\
\hline 2b & Ball Stern & & \\
\hline 2c & Baum Stern & & \\
\hline 2d & Schuh Ball & & \\
\hline
\end{tabular}

\begin{tabular}{|l|l|l|l|}
\hline 3a & Schuh Haus Topf & & \\
\hline 3b & Baum Ball Haus & & \\
\hline 3c & Eis Schuh Pilz & & \\
\hline 3d & Schuh Baum Ball & & \\
\hline
\end{tabular}

\begin{tabular}{|l|l|}
\hline 4a & Topf Baum Schuh Ball \\
\hline 4b & Haus Pilz Stern Baum \\
\hline 4c & Fisch Eis Ball Stern \\
\hline 4d & Pilz Schuh Topf Baum \\
\hline
\end{tabular}

\begin{tabular}{|l|l|l|l|}
\hline $\mathbf{5 a}$ & Schuh Stern Pilz Eis Topf & & \\
\hline $\mathbf{5 b}$ & Haus Ball Fisch Topf Baum & & \\
\hline $\mathbf{5 c}$ & Stern Topf Ball Haus Eis & & \\
\hline $\mathbf{5 d}$ & Topf Fisch Baum Pilz Haus & & \\
\hline
\end{tabular}

5d Topf Fisch Baum Pilz Haus

\begin{tabular}{|l|l|l|l|}
\hline $\mathbf{6 a}$ & Stern Pilz Ball Topf Eis Schuh & & \\
\hline $\mathbf{6 b}$ & Baum Schuh Haus Pilz Stern Ball & & \\
\hline $\mathbf{6 c}$ & Ball Haus Eis Baum Stern Schuh & & \\
\hline 6d & Baum Schuh Pilz Topf Stern Eis & & \\
\hline
\end{tabular}

\begin{tabular}{|l|l|l|l|}
\hline 7a & Haus Baum Topf Fisch Pilz Ball Eis & & \\
\hline 7b & Ball Pilz Haus Baum Fisch Topf Stern & & \\
\hline 7c & Haus Pilz Stern Baum Schuh Ball Topf & & \\
\hline 7d & Schuh Eis Haus Topf Pilz Baum Ball & & \\
\hline
\end{tabular}

\begin{tabular}{|l|l|l|l|}
\hline $\mathbf{8 a}$ & Haus Stern Ball Pilz Baum Schuh Fisch Eis & & \\
\hline $\mathbf{8 b}$ & Schuh Pilz Haus Ball Stern Fisch Topf Baum & & \\
\hline $\mathbf{8 c}$ & Eis Ball Schuh Topf Stern Baum Fisch Pilz & & \\
\hline & $\begin{array}{l}\text { Stern Schuh Ball Baum Eis Topf Haus } \\
\text { Fisch }\end{array}$ & & \\
$\mathbf{8 d}$ & RW: & \\
\hline \multicolumn{4}{|r}{}
\end{tabular}

\subsection{Dreisilbige Wörter}

\begin{tabular}{|c|c|c|c|}
\hline & Reiz & $\mathrm{k}$ & $\mathrm{ik}$ \\
\hline $2 a$ & Fernseher Erdbeere & & \\
\hline $2 \mathbf{b}$ & Eisenbahn Lichtschalter & & \\
\hline 2c & Briefkasten Lichtschalter & & \\
\hline 2d & Kneifzange Eisenbahn & & \\
\hline $3 a$ & Kneifzange Zahnbürste Fernseher & & \\
\hline $3 b$ & Briefkasten Eisenbahn Zahnbürste & & \\
\hline $3 c$ & Erdbeere Kneifzange Schaukelpferd & & \\
\hline 3d & Kneifzange Briefkasten Eisenbahn & & \\
\hline $4 a$ & Fernseher Briefkasten Kneifzange Eisenbahn & & \\
\hline $4 b$ & Zahnbürste Schaukelpferd Lichtschalter Briefkasten & & \\
\hline 4c & Luftballon Erdbeere Eisenbahn Lichtschalter & & \\
\hline 4d & Schaukelpferd Kneifzange Fernseher Briefkasten & & \\
\hline $5 a$ & Kneifzange Lichtschalter Schaukelpferd Erdbeere Fernseher & & \\
\hline $5 b$ & Zahnbürste Eisenbahn Luftballon Fernseher Briefkasten & & \\
\hline $5 c$ & Lichtschalter Fernseher Eisenbahn Zahnbürste Erdbeere & & \\
\hline 5d & Fernseher Luftballon Briefkasten Schaukelpferd Zahnbürste & & \\
\hline $6 a$ & Lichtschalter Schaukelpferd Eisenbahn Fernseher Erdbeere Kneifzange & & \\
\hline $6 b$ & Briefkasten Kneifzange Zahnbürste Schaukelpferd Lichtschalter Eisenbahn & & \\
\hline $6 c$ & Eisenbahn Zahnbürste Erdbeere Briefkasten Lichtschalter Kneifzange & & \\
\hline 6d & Briefkasten Kneifzange Schaukelpferd Fernseher Lichtschalter Erdbeere & & \\
\hline $7 a$ & Zahnbürste Briefkasten Fernseher Luftballon Schaukelpferd Eisenbahn Erdbeere & & \\
\hline $7 \mathbf{b}$ & Eisenbahn Schaukelpferd Zahnbürste Briefkasten Luftballon Fernseher Lichtschalter & & \\
\hline 7c & Zahnbürste Schaukelpferd Lichtschalter Briefkasten Kneifzange Eisenbahn Fernseher & & \\
\hline 7d & Fernseher Zahnbürste Kneifzange Briefkasten Schaukelpferd Erdbeere Eisenbahn & & \\
\hline $8 \mathbf{a}$ & Zahnbürste Lichtschalter Eisenbahn Schaukelpferd Briefkasten Kneifzange Luftballon Erdbeere & & \\
\hline $8 \mathbf{b}$ & Kneifzange Schaukelpferd Zahnbürste Eisenbahn Lichtschalter Luftballon Fernseher Briefkasten & & \\
\hline $8 c$ & Erdbeere Eisenbahn Kneifzange Fernseher Lichtschalter Briefkasten Luftballon Schaukelpferd & & \\
\hline $8 d$ & $\begin{array}{l}\text { Lichtschalter Kneifzange Eisenbahn Briefkasten Erdbeere } \\
\text { Fernseher Zahnbürste Luftballon }\end{array}$ & & \\
\hline
\end{tabular}




\section{Corsi-Blocks}

\subsection{Sequenz einfach}

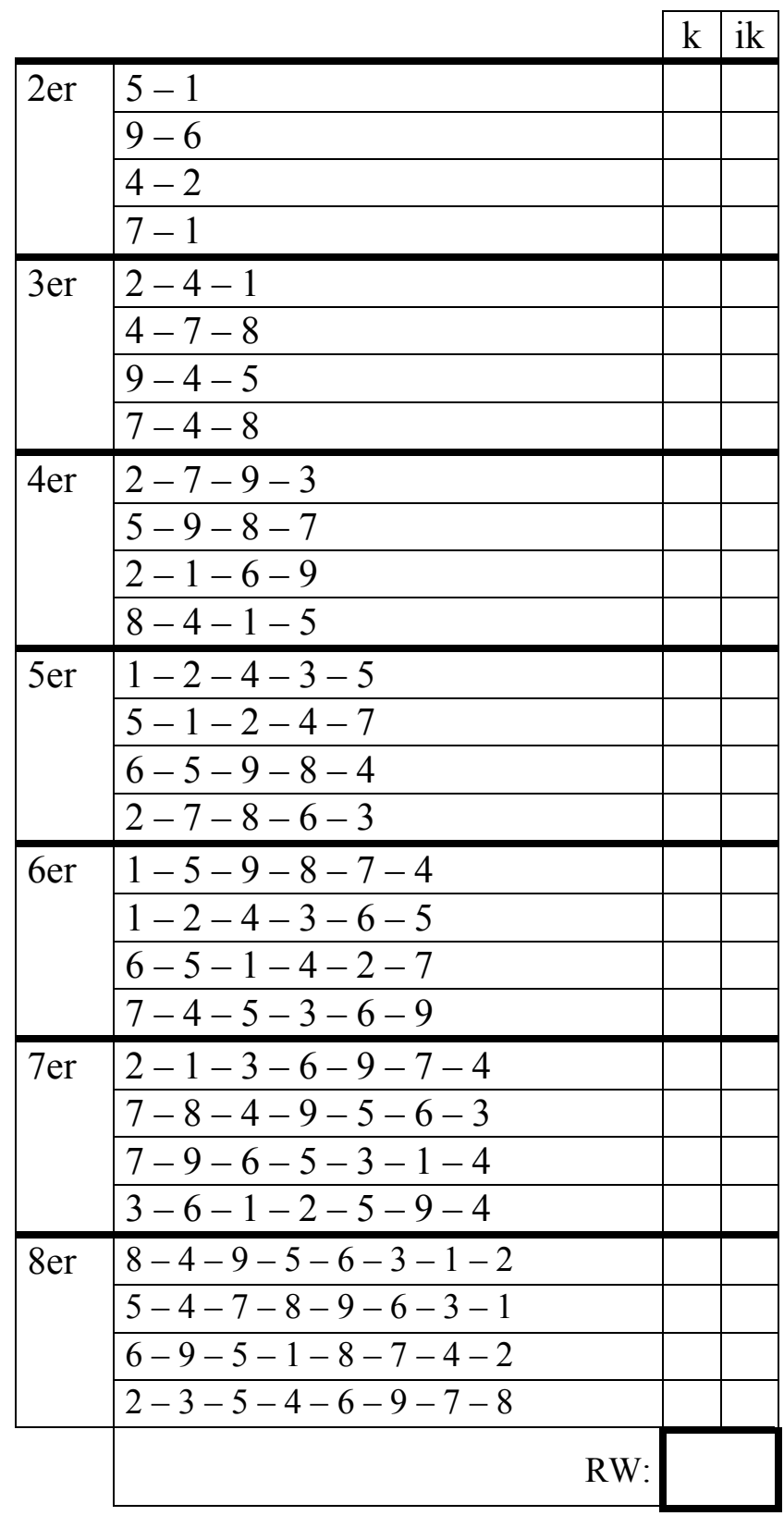

\subsection{Sequenz komplex}

\begin{tabular}{|c|c|c|c|}
\hline & & $\mathrm{k}$ & $\mathrm{ik}$ \\
\hline 2er & $3-8$ & & \\
\hline & $2-6$ & & \\
\hline & $7-1$ & & \\
\hline & $9-2$ & & \\
\hline 3 er & $9-1-7$ & & \\
\hline & $8-2-3$ & & \\
\hline & $7-6-4$ & & \\
\hline & $3-9-2$ & & \\
\hline 4 er & $4-6-7-3$ & & \\
\hline & $6-4-1-9$ & & \\
\hline & $8-1-9-2$ & & \\
\hline & $2-3-8-1$ & & \\
\hline $5 \mathrm{er}$ & $9-3-5-6-4$ & & \\
\hline & $6-4-3-8-5$ & & \\
\hline & $2-8-6-7-3$ & & \\
\hline & $7-5-8-1-6$ & & \\
\hline $6 e r$ & $5-7-6-4-3-8$ & & \\
\hline & $1-6-4-3-8-5$ & & \\
\hline & $3-7-6-2-1-8$ & & \\
\hline & $7-6-1-8-5-2$ & & \\
\hline 7er & $2-5-8-6-4-3-9$ & & \\
\hline & $7-3-9-2-8-6-4$ & & \\
\hline & $5-9-4-6-3-1-8$ & & \\
\hline & $8-4-7-9-1-2-6$ & & \\
\hline 8 er & $4-6-7-3-2-9-1-8$ & & \\
\hline & $6-2-9-3-8-1-7-5$ & & \\
\hline & $5-2-1-8-7-6-3-4$ & & \\
\hline & $9-3-6-4-5-8-1-7$ & & \\
\hline & RW: & & \\
\hline
\end{tabular}

Tafel 3.1 zur Präsentation verwenden. 
4. Nonword Repetition A

\subsection{Nonwords Version A}

\begin{tabular}{|l|l|l|}
\hline \multicolumn{1}{|c|}{ Nonword } & $\mathbf{k}$ & $\mathbf{i k}$ \\
\hline Beispiel 1: bralzen & & \\
\hline Beispiel 2: gliebunade & & \\
\hline Beispiel 3: franulich & & \\
\hline
\end{tabular}

\begin{tabular}{|l|l|l|}
\hline werulater & & \\
\hline wuralten & & \\
\hline
\end{tabular}

maling

zawo

schliebunder

vorluch

verkraffern

sulibritzen

fallurwakel

bestrugeln

(p)fitzorkaden

lefal

krauern

nalos

geschraken

kattaus

mindinnen

saberlicke

(p)franden

karflumen

jalosse

kudilabert

verkrabaten

arbaklaschen

RW (max. 24) 


\section{Gedächtnisspanne rückwärts}

\subsection{Wörter rückwärts}

\begin{tabular}{|c|c|c|}
\hline & Reiz & \begin{tabular}{|l|l|}
$\mathrm{k}$ & $\mathrm{ik}$ \\
\end{tabular} \\
\hline $2 a$ & Schuh Stern & \\
\hline $2 \mathbf{b}$ & Ball Topf & \\
\hline $2 \mathrm{c}$ & Ball Eis & \\
\hline 2d & Topf Fisch & \\
\hline $3 a$ & Stern Baum Fisch & \\
\hline $3 \mathbf{b}$ & Baum Topf Eis & \\
\hline $3 c$ & Haus Fisch Schuh & \\
\hline 3d & Topf Eis Fisch & \\
\hline $4 a$ & Topf Fisch Eis Stern & \\
\hline $4 \mathrm{~b}$ & Eis Ball Haus Baum & \\
\hline $4 c$ & Ball Topf Schuh Pilz & \\
\hline 4d & Eis Stern Fisch Haus & \\
\hline $5 \mathbf{a}$ & Stern Schuh Haus Ball Fisch & \\
\hline $5 b$ & Eis Stern Pilz Topf Baum & \\
\hline $5 c$ & Schuh Baum Topf Stern Ball & \\
\hline 5d & Baum Haus Eis Pilz Stern & \\
\hline $6 a$ & Fisch Schuh Stern Topf Haus Ball & \\
\hline $6 \mathrm{~b}$ & Topf Ball Haus Baum Fisch Eis & \\
\hline $6 c$ & Fisch Ball Eis Schuh Baum Topf & \\
\hline 6d & Schuh Ball Stern Haus Fisch Eis & \\
\hline $7 a$ & Schuh Topf Haus Pilz Stern Eis Baum & \\
\hline $7 \mathrm{~b}$ & Ball Stern Pilz Eis Baum Haus Topf & \\
\hline $7 \mathrm{c}$ & Stern Topf Fisch Eis Ball Haus Baum & \\
\hline $7 \mathrm{~d}$ & Topf Schuh Haus Eis Fisch Baum Stern & \\
\hline $8 \mathbf{a}$ & Schuh Pilz Fisch Eis Haus Topf Ball Baum & \\
\hline $8 b$ & Eis Stern Pilz Ball Topf Baum Haus Fisch & \\
\hline $8 \mathrm{c}$ & Haus Pilz Eis Ball Stern Fisch Topf Schuh & \\
\hline $8 \mathrm{~d}$ & Pilz Baum Stern Schuh Eis Topf Fisch Ball & \\
\hline
\end{tabular}

\subsection{Ziffern rückwärts}

\begin{tabular}{|l|l|l|l|}
\hline & Reiz & k & ik \\
\hline $\mathbf{2 a}$ & $1-8$ & & \\
\hline $\mathbf{2 b}$ & $7-4$ & & \\
\hline $\mathbf{2 c}$ & $7-2$ & & \\
\hline $\mathbf{2 d}$ & $4-9$ & & \\
\hline \multicolumn{3}{|l|}{} \\
\hline $\mathbf{3 a}$ & $8-6-9$ & & \\
\hline $\mathbf{3 b}$ & $6-4-2$ & & \\
\hline $\mathbf{3 c}$ & $3-9-1$ & & \\
\hline $\mathbf{3 d}$ & $4-2-9$ & & \\
\hline \multicolumn{2}{|l|}{} \\
\end{tabular}

\begin{tabular}{|l|l|l|l|}
\hline $\mathbf{6 a}$ & $9-1-8-4-3-7$ & & \\
\hline $\mathbf{6 b}$ & $4-7-3-6-9-2$ & & \\
\hline $\mathbf{6 c}$ & $9-7-2-1-6-4$ & & \\
\hline $\mathbf{6 d}$ & $1-7-8-3-9-2$ & & \\
\hline &
\end{tabular}

7a 1 - $1-4-3-5-8-2-6$

7b $7-8-5-2-6-3-4$

7c $8-4-9-2-7-3-6$

7d $4-1-3-2-9-6-8$

8a 1 - 5 - 9-2 - 3-4-7-6

8b $2-8-5-7-4-6-3-9$

8c $3-5-2-7-8-9-4-1$

8d $5-6-8-1-2-4-9-7$

RW: 
6. Counting Span

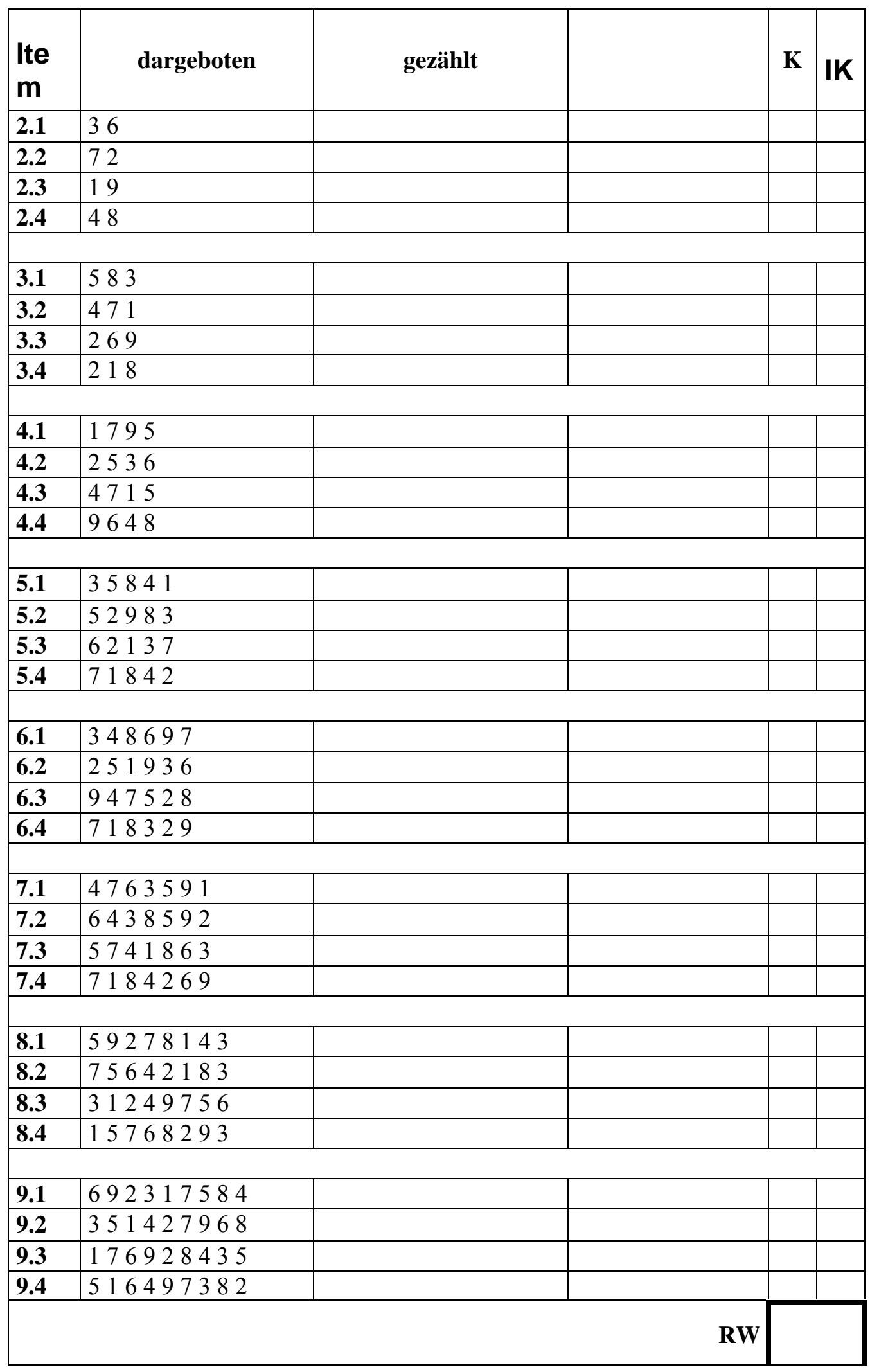




\section{Ziffernspanne vorwärts}

\begin{tabular}{|c|c|c|c|}
\hline & Reiz & $\mathrm{k}$ & $\mathrm{ik}$ \\
\hline $2 a$ & $4-2$ & & \\
\hline $2 b$ & $7-8$ & & \\
\hline $2 c$ & $6-8$ & & \\
\hline $2 d$ & $1-7$ & & \\
\hline $3 \mathbf{a}$ & $1-3-4$ & & \\
\hline $3 b$ & $6-7-3$ & & \\
\hline $3 c$ & $2-1-5$ & & \\
\hline $3 d$ & $1-6-7$ & & \\
\hline $4 a$ & $4-6-1-7$ & & \\
\hline $4 b$ & $3-5-8-6$ & & \\
\hline 4c & $9-2-7-8$ & & \\
\hline 4d & $5-1-4-6$ & & \\
\hline $5 a$ & $1-8-5-2-4$ & & \\
\hline $5 b$ & $3-7-9-4-6$ & & \\
\hline $5 c$ & $8-4-7-3-2$ & & \\
\hline $5 d$ & $4-9-6-5-3$ & & \\
\hline $6 \mathbf{a}$ & $8-5-7-4-2-1$ & & \\
\hline $6 b$ & $6-1-3-5-8-7$ & & \\
\hline $6 c$ & $7-3-2-6-8-1$ & & \\
\hline $6 d$ & $6-1-5-4-8-2$ & & \\
\hline $7 a$ & $3-6-4-9-5-7-2$ & & \\
\hline $7 b$ & $7-5-3-6-9-4-8$ & & \\
\hline $7 c$ & $3-5-8-6-1-7-4$ & & \\
\hline $7 d$ & $4-3-1-6-5-2-7$ & & \\
\hline $8 \mathbf{a}$ & $3-8-7-5-6-1-9-2$ & & \\
\hline $8 b$ & $1-5-3-7-8-9-4-6$ & & \\
\hline $8 c$ & $2-7-1-4-8-6-9-5$ & & \\
\hline $8 d$ & $8-1-7-6-2-4-3-9$ & & \\
\hline
\end{tabular}

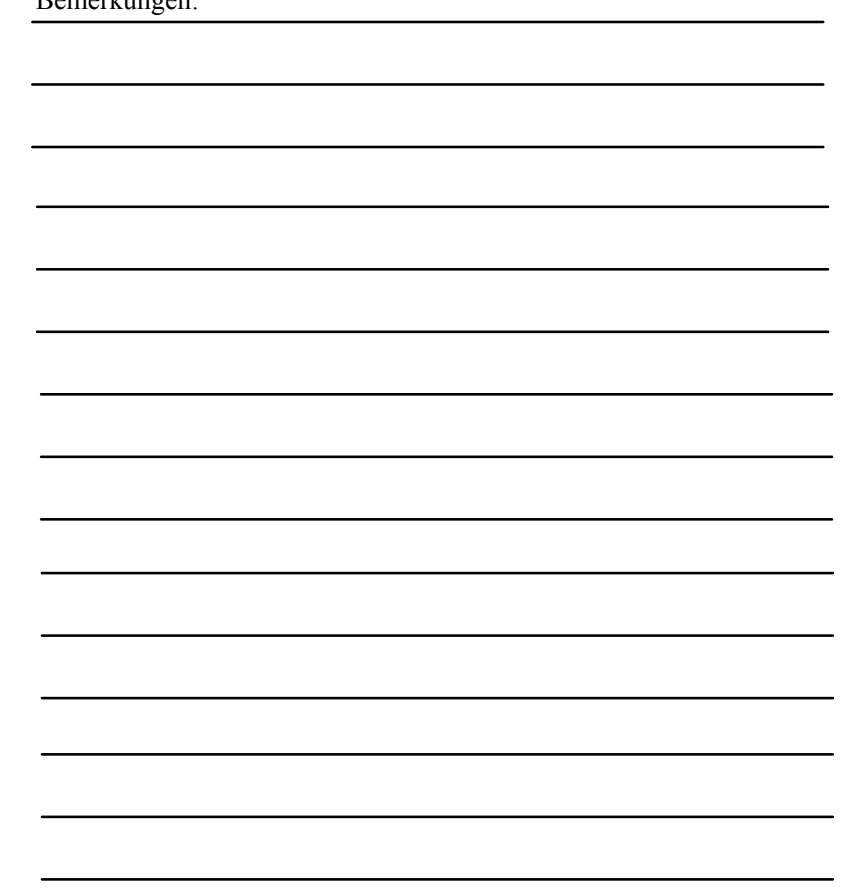


8. Matrix

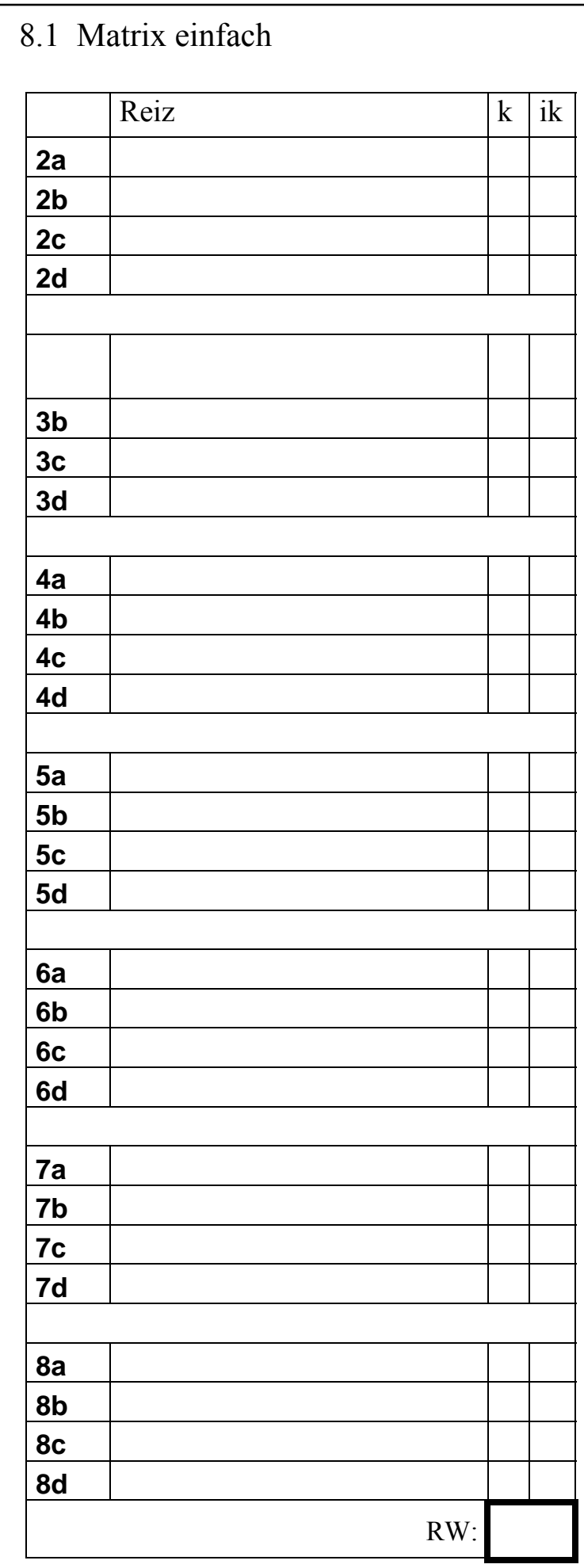

Tafel zur Kontrolle verwenden.

\subsection{Matrix komplex}

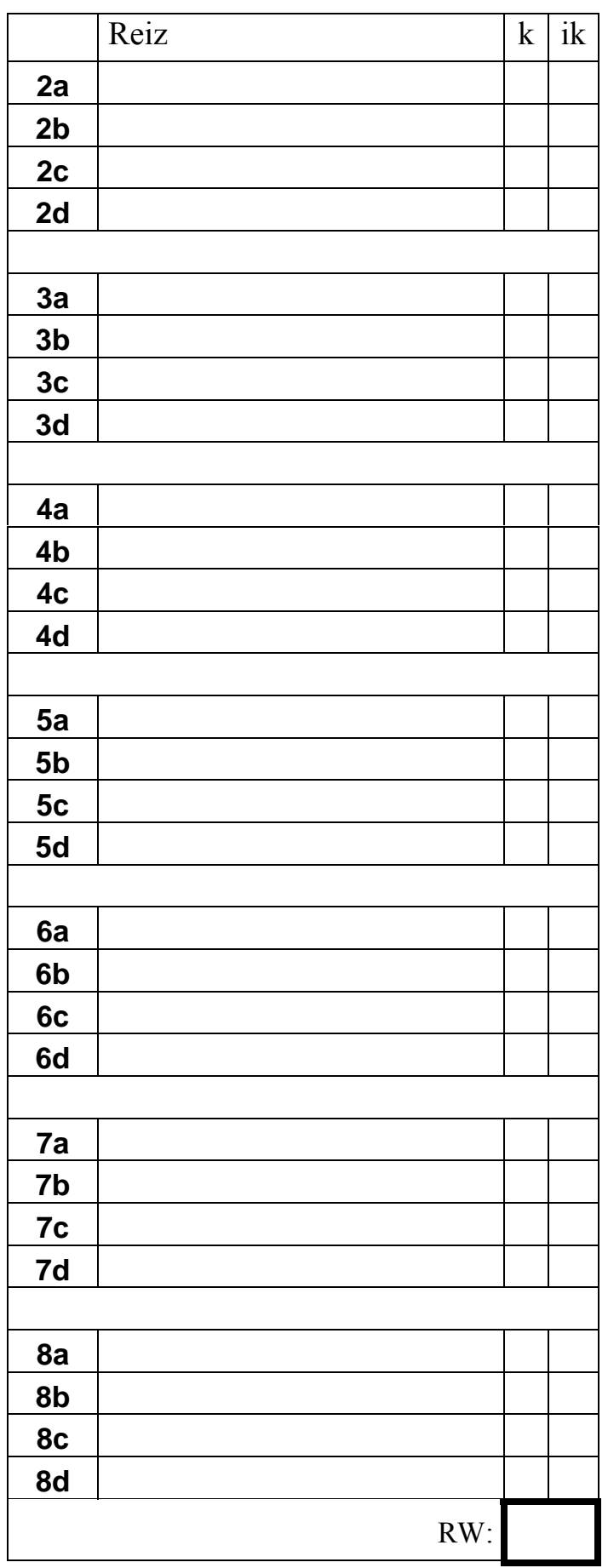




\section{Gedächtnisspanne Nonwords}

\section{1 einsilbige Wörter}

\begin{tabular}{|l|l|l|l|}
\hline & Reiz & $\mathrm{k}$ & $\mathrm{ik}$ \\
\hline 2a & fen sim & & \\
\hline $\mathbf{2 b}$ & bork deil & & \\
\hline 2c & laum natt & & \\
\hline 2d & tirk fen & & \\
\hline & & & \\
\hline 3a & deil sim bork & & \\
\hline 3b & sim tirk laum & & \\
\hline 3c & natt bork fen & & \\
\hline 3d & tirk bork natt & & \\
\hline & & & \\
\hline 4a & bork laum deil fen & & \\
\hline 4b & laum sim fen tirk & & \\
\hline 4c & bork sim natt laum & & \\
\hline 4d & sim deil tirk fen & & \\
\hline & & & \\
\hline 5a & natt bork laum tirk deil & & \\
\hline 5b & tirk fen natt bork sim & & \\
\hline 5c & deil sim tirk bork fen & & \\
\hline 5d & sim laum deil bork natt & & \\
\hline & & & \\
\hline 6a & deil laum fen sim tirk bork & & \\
\hline 6b & sim deil natt laum bork fen & & \\
\hline 6c & tirk natt sim deil bork laum & & \\
\hline 6d & laum tirk fen bork natt deil & & \\
\hline & & & \\
\hline 7a & natt laum tirk deil bork sim fen & & \\
\hline 7b & fen sim bork deil natt laum tirk & & \\
\hline 7c & tirk bork fen deil sim laum natt & & \\
\hline 7d & deil fen laum sim tirk natt bork & & \\
\hline & & \\
\hline & & \\
\hline
\end{tabular}

\section{2 dreisilbige Wörter}

\begin{tabular}{|c|c|c|}
\hline & Reiz & $\mathrm{k}$ \\
\hline $2 \mathbf{a}$ & franulich schleibunder & \\
\hline 2b & bestrugeln reseubelt & \\
\hline 2c & külinge mindinnen & \\
\hline 2d & laujossung franulich & \\
\hline 3a & reseubelt schleibunder bestrugeln & \\
\hline $3 \mathbf{b}$ & schleibunder laujossung külinge & \\
\hline $3 c$ & mindinnen bestrugeln franulich & \\
\hline 3d & laujossung bestrugeln mindinnen & \\
\hline $4 \mathbf{a}$ & bestrugeln külinge reseubelt franulich & \\
\hline $4 \mathbf{b}$ & külinge schleibunder franulich laujossung & \\
\hline 4c & bestrugeln schleibunder mindinnen külinge & \\
\hline 4d & schleibunder reseubelt laujossung franulich & \\
\hline $5 \mathbf{a}$ & mindinnen bestrugeln külinge laujossung reseubelt & \\
\hline $5 \mathbf{b}$ & laujossung franulich mindinnen bestrugeln schleibunder & \\
\hline $5 c$ & reseubelt schleibunder laujossung bestrugeln franulich & \\
\hline 5d & schleibunder külinge reseubelt bestrugeln mindinnen & \\
\hline 6a & reseubelt külinge franulich schleibunder laujossung bestrugeln & \\
\hline $6 \mathbf{b}$ & schleibunder reseubelt mindinnen külinge bestrugeln franulich & \\
\hline 6c & laujossung mindinnen schleibunder reseubelt bestrugeln külinge & \\
\hline 6d & külinge laujossung franulich bestrugeln mindinnen reseubelt & \\
\hline $7 \mathbf{a}$ & mindinnen külinge laujossung reseubelt bestrugeln schleibunder franulich & \\
\hline $7 \mathbf{b}$ & franulich schleibunder bestrugeln reseubelt mindinnen külinge laujossung & \\
\hline 7c & laujossung bestrugeln franulich reseubelt schleibunder külinge mindinnen & \\
\hline 7d & reseubelt franulich külinge schleibunder laujossung mindinnen bestrugeln & \\
\hline
\end{tabular}

